\title{
ZWISCHENEVALUIERUNG MIKROTECHNIK ÖSTERREICH
}

KLAUS ZINÖCKER, MICHAEL DINGES, ANDREAS SCHIBANY

unter Mitarbeit von

Julia Schindler, Julia Schmidmayer, Franziska Steyer 


\section{ZWISCHENEVALUIERUNG MIKROTECHNIK ÖSTERREICH}

Im Auftrag des Forschungsförderungsfonds für die gewerbliche Wirtschaft - FFF

KLAUS ZINÖCKER, MICHAEL DINGES, ANDREAS SCHIBANY

unter Mitarbeit von

Julia Schindler, Julia Schmidmayer, Franziska Steyer

Wien, November 2004

Projekt No. RTW.2003.AF.023-01

Joanneum Research Forschungsgesellschaft mbH

Institut für Technologie- und Regionalpolitik (InTeReg)

Wiedner Hauptstraße 76, 1040 Wien

Tel. +43-1-581 7520 und

Elisabethstraße 17, 8010 Graz

Tel. $+43-316-8761488$ 


\section{Evaluierungsergebnis in $\mathbf{3 0}$ Sekunden}

Mit der Initiative Mikrotechnik wählt der FFF einen richtigen Zugang zur technologischen Schwerpunktsetzung, da er einen Bereich fokussiert, der als Basistechnologie eingeschätzt wird und für die österreichische Wirtschaft von Bedeutung ist. Die Evaluatoren sehen keinen Grund, diesen Weg nicht fortzusetzen.

Die Mission des Programms, „Verankerung und Weiterentwicklung von Mikrotechnik in Österreich auf breiter Basis - insbesondere in KMU“, konnte allerdings nur teilweise erreicht werden. Als positiv anzusehen ist eine hohe technologische Qualität der Projekte, als negativ die kaum in Anspruch genommene Vernetzung mit anderen Förderinstrumenten des FFF.

Das Evaluierungsteam sieht Verbesserungsmöglichkeiten im Programmmanagement und in einer engeren Zusammenarbeit mit der Nano - Initiative Austria. Wir empfehlen daher, die Initiative Mikrotechnik unter diesen Gesichtspunkten einem Re-Design zu unterziehen. 


\section{Evaluierung der Initiative Mikrotechnik - Zusammenfassung in Schlagzeilen}

\section{Schwerpunkt Mikrotechnik}

- Mit der Initiative Mikrotechnik hat der FFF ein Technologiefeld aufgegriffen, das als Basistechnologie gesehen wird und dessen Bedeutung für österreichische Unternehmen den Einsatz von öffentlichen Mitteln lohnt. Der Platz von Mikro- und Nanotechnologie auf der österreichischen Forschungs- und technologiepolitischen Agenda ist gerechtfertigt und entspricht den Orientierungspunkten, die der Rat für Forschung und Technologieentwicklung für die Akteure des nationalen Innovationssystems gesetzt hat.

- Mit der Fokussierung auf Mikro- und Nanotechnologie hat der FFF einen richtigen Zugang zur Setzung von Schwerpunkten in seiner Tätigkeit gewählt.

- Aus Sicht der Evaluatoren gibt es keine Gründe, den Schwerpunkt Mikrotechnik nicht fortzuführen.

\section{Zielsystem}

- Die Initiative Mikrotechnik ist in ihrem Zielsystem, in den gewählten Maßnahmen und den intendierten Wirkungen weitgehend konsistent.

\section{Die Initiative Mikrotechnik hat evaluierbare Leitkriterien}

- Die Initiative Mikrotechnik hat klare, evaluierbare Leitkriterien. Das Formulieren solcher Kriterien war immer wieder eine Forderung an die Akteure der Forschungs- und Technologiepolitik, um Maßnahmen nachvollzieh- und einschätzbarer (evaluierbarer) zu machen und so zu einer besseren, rationaleren Maßnahmenplanung beizutragen. Mit der Formulierung dieser Leitkriterien hat der FFF diesbezüglich ein Zeichen gesetzt und internationalen ,good practice“ Standards entsprochen.

\section{Förderempfänger und Projekte}

- In der Initiative Mikrotechnik haben 65 Unternehmen und 7 Arbeitsgemeinschaften insgesamt 150 Förderanträge eingebracht, von denen 110 (73\%) bewilligt wurden.

- Von den 150 eingereichten Projekten wurden 105 der Mikrotechnologie und 45 der Nanotechnologie zugeordnet.

- Im Gegensatz zur Gesamtheit der FFF Projekte zeichnen sich die Projekte der Initiative Mikrotechnik durch deutlich höhere Projektkosten aus. Die Förderquote (Förderbarwert im Verhältnis zu den Projektkosten) liegt im üblichen Bereich des FFF - Fördermixes.

- Die Mehrzahl der Förderanträge befindet sich in der Gruppe der Großprojekte mit mehr als $500.000 €$ Gesamtprojektvolumen. Diese Verteilung weicht klar von der Gesamtverteilung der FFF-Projekte ab. 


\section{Förderempfänger: Profis oder Newcomer?}

- Auf jene 6 Unternehmen, die am häufigsten um eine FFF-Förderung in der Initiative Mikrotechnik angesucht haben, entfallen 37\% der Projektanträge und 33\% der durchgeführten Projekte. Sie lukrieren 46,7\% der gesamten Fördermittel und 46,4\% des Förderbarwertes.

- Lediglich 9 Unternehmen und 5 ARGEs haben ausschließlich einen Förderantrag in der Initiative Mikrotechnik eingereicht.

- In der Initiative Mikrotechnik spielen sehr aktive Förderwerber eine dominante Rolle.

- Die Antragssteller der Initiative Mikrotechnik sind deutlich aktivere Förderwerber als die Gesamtheit der Antragssteller beim FFF.

\section{Kooperation Wissenschaft - Wirtschaft}

- Von den 110 durchgeführten Projekten der Initiative Mikrotechnik wurden 27\% in Kooperation mit Forschungseinrichtungen betrieben.

\section{Nutzung anderer Aktionslinien}

- Die Initiative Mikrotechnik greift andere Aktionslinien des FFF auf und regt zu deren Nutzung an. Nicht in jedem Programm, in jeder Initiative das Rad neu erfinden zu wollen, ist begrüßenswert und effizient. Nun ist aber festzustellen, dass die Aktionslinien ,Feasibility Studies' und ,F\&E Dynamik' im Bereich Mikrotechnik kaum bzw. gar nicht genutzt werden: Es wurden lediglich 3 Feasibility Studies durchgeführt, die Aktionslinie F\&E Dynamik wurde nicht genutzt.

\section{Positionierung zur Nano Initiative der ASA}

- Die Initiative Mikrotechnik dupliziert die Nano-Initiative der ASA nicht, es ist jedoch ein Potential der wechselseitigen Verstärkung erkennbar; gleichzeitig dupliziert jedoch die Nano Initiative in Teilbereichen das Tätigkeitsspektrum des FFF..

\section{Qualität der Daten}

- Der FFF verfügt über ausreichend Datenmaterial, um eine Charakterisierung der partizipierenden Unternehmen vorzunehmen. Sowohl auf der Projektebene als auch auf der Ebene der beteiligten Akteure ist allerdings eine deutliche Verbesserung der Monitoring-Aktivitäten zu empfehlen - vor allem sollten jene Informationen gesammelt werden, die für eine Einschätzung der Leitkriterien notwendig sind.

\section{Begutachtung der Projekte}

- Im Begutachtungsprozess bevorzugt der FFF Mikrotechnik Projekte gegenüber anderen Projekten nicht. Auch macht der Fonds keine Unterscheidungen zwischen Projekten der Mikro- und Nanotechnologie. 


\section{Zielerreichung: Leitkriterien}

- Das Leitkriterium 100-150 Projektanträge in der Initiative Mikrotechnik konnte mit 150 Projektanträgen am oberen Ende der Erwartungen erfüllt werden. Die Anzahl der förderwerbenden Unternehmen entsprach ebenso der Zielgröße.

\section{Wurde das Technologiefeld „Mikrotechnik“bzw „Nanotechnik“ in Österreich durch den} FFF verankert und weiterentwickelt?

- Insgesamt wickelte der FFF 110 Projekte im Feld „Mikro- und Nanotechnik“ mit einem Volumen von rund 55 Mio Euro ab. Damit hat der FFF qua Programmexistenz einen Beitrag zur Verankerung und Weiterentwicklung geleistet. Die Bedeutung bzw. der Mehrwert der Initiative kann jedoch zum gegenwärtigen Zeitpunkt nicht seriös eingeschätzt werden.

\section{Geschah dies auf der intendierten breiten Basis?}

- Seit dem 3 jährigen Bestsehen der Initiative Mikrotechnik reichten 65 Unternehmen insgesamt 150 Projektanträge ein. Damit konnten die in den Leitkriterien angestrebten Werte erreicht werden. Dieses durchaus positive Bild wird von der Tatsache konterkariert, dass eine vergleichsweise geringe Anzahl von Unternehmen (sechs) beinahe die Hälfte der vergebenen Fördermittel lukrierten.

\section{Wurden insbesondere KMU angesprochen?}

- Der FFF hat sich mit der Initiative Mikrotechnik ambitioniert das Ziel gesetzt, insbesondere KMU anzusprechen. Jedoch wurden in der Initiative insgesamt weniger KMU angesprochen als im gesamten Fördergeschäft des FFF. 63\% der Anträge und 41\% der Projekte in der Initiative Mikrotechnik stammten von KMU, allerdings lukrierten große Unternehmen mit mehr als 250 MitarbeiterInnen 74,4\% der Fördermittel. Laut dem Jahresbericht des FFF 2002 sind 80\% aller Antragssteller KMUs; 50 \% der Fördermittel fließen in kleine und mittlere Unternehmen.

\section{Wurden kritische Massen erreicht?}

Die Antwort auf diese Frage ist zweizuteilen:

- Nein, auf Grund der niedrigen Zahl der Neukunden (lediglich 9 Unternehmen haben sich ausschließlich für Projekte innerhalb der Initiative Mikrotechnik beworben) können wir nicht davon ausgehen, dass die Initiative Mikrotechnik einen Beitrag dazu geleistet hat, dass kritische Massen entstehen.

- Nein, denn aufgrund der sehr schiefen Verteilung der gesamten Fördervolumina für Großunternehmen wurden keine zusätzlichen Kunden angesprochen, sondern ohnehin starke Unternehmen, die in ihren Kernkompetenzen agieren, gestärkt. (siehe Verteilung der Mittel)

- Konterkariert wird dieses Bild von folgender Überlegung: Auf Grund der hohen Volumen des Programms (über 55 Millionen Euro) und der Ergebnisse der FFF-Evaluierung können wir davon ausgehen, dass der FFF dazu beigetragen hat, dass Österreichische Unternehmen im Bereich Mikro- und Nanotechnologie mehr entwickeln. Auch können durch die Initiative Veränderungen der Forschungsportfolios in Großunternehmen (in 
Richtung Mikro- und Nanotechnologie) angeregt worden sein, allerdings können wir hierzu keine verlässlichen Aussagen treffen. Eine weitere Möglichkeit, zur Erreichung von kritischen Massen beizutragen ist die Bündelung von Forschungsaktivitäten. Wir können hierzu auf Basis der Daten keine Aussage treffen.

\section{Wurde zur Kooperation „Wissenschaft - Wirtschaft“ beigetragen?}

Der in den Leitkriterien angepeilte Wert von 30\% Wissenschaft-Wirtschaft Kooperationen konnte knapp nicht erreicht werden. Der FFF setzt zwar Anreize für Kooperationen (etwa über einen höheren Förderbarwert), stellt das nach außen hin jedoch nicht genügend dar.

Auf Grund der nur unbedeutend unterschiedlichen Ablehnungsrate zwischen Projekten „mit“ und „ohne“ Kooperationen kann keine Aussage getroffen werden, ob die Initiative Mikrotechnik einen zusätzlichen Kooperationsimpuls geleistet hat.

\section{Internationalisierung}

Über die in den Leitkriterien gesetzten Ziele von grenzüberschreitenden Kooperationen können wir aufgrund des fehlenden Datenmaterials keine Angaben machen. Die Internationalisierung des FFF über die Initiative Mikrotechnik erfolgte über ein ERA-NET Projekt, das unter der Leitung des FFF steht. Dieses soll in der Zukunft als Ansatzpunkt dienen, um auch eine internationale Vernetzung von Unternehmen und Forschungseinrichtungen zu ermöglichen.

\section{Additionalität}

Wir glauben, dass die Initiative Mikrotechnik - auf Grund des Design des Programms (keine zusätzlichen Budgetmittel, Zuordnung von Projekten) nur wenig additionale Effekte ausgelöst hat. Auch die Ausführungen in Bezug auf die flankierenden Maßnahmen unterstreichen das. Für die Projekte sind ähnliche Aussagen wie bei der FFF/FWF Evaluierung zu treffen: „Die FFF-Förderung zeigt eine positive Hebelwirkung auf die unternehmensinternen F\&E-Aufwendungen: der Barwert einer Fördereinheit bewirkt zusätzliche private F\&E-Aufwendungen von 0,4 Einheiten.“

\section{Programmspezifika}

Internationale Vergleiche zeigen, dass die Charakteristika von Technologieprogrammen sich dahingehend verändert haben, als neben der Entwicklung neuer Technologien hinaus auch weitere forschungs- und innovationspolitischen Zielsetzungen verfolgt und auch dementsprechende (flankierende) Maßnahmen (im Falle von Mikrotechnik waren dies einige wenige Awarenessmaßnahmen wie die Roadshow oder die Etablierung eines Lenkungsausschusses) gesetzt werden. Eine inhaltliche Zusammenfassung von bottom-up Projekten macht noch kein Programm aus.

Daher ist u.E. nach die Schnittstelle zwischen der (von der FFF Evaluierung sehr positiv bewerteten) bottom-up Förderung und den Charakteristika und Spezifika eines Technologieförderprogramms Mikrotechnik nicht klar erkennbar. 


\section{Handlungsoptionen}

Wir empfehlen, dass der FFF bei der Initiative Mikrotechnik ein grundlegendes Redesign vornimmt. Der FFF muss, gemeinsam mit anderen Akteuren innerhalb der neu gegründeten FFG, entscheiden, ob dieses Redesign unter einem gemeinsamen Dach „Mikro- und Nanotechnologie Österreich“" passiert oder ob der FFF in Abstimmung mit der Nano Initiative die Aktionslinie Mikrotechnik neu aufsetzt. 


\section{Inhaltsverzeichnis}

1 EINLEITUNG.

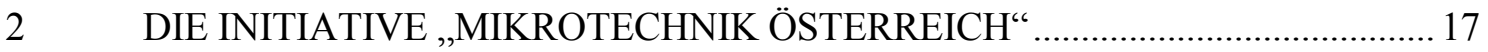

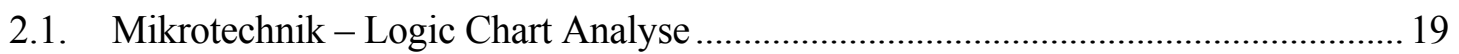

2.2. Mission und Zielsetzung der Initiative Mikrotechnik ............................................... 22

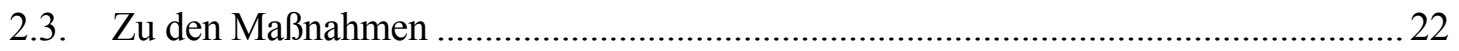

2.4. Zu den intendierten Wirkungen.......................................................................... 23

3 QUANTITATIVE ANALYSE DER MIKROTECHNIK INITIATIVE................... 26

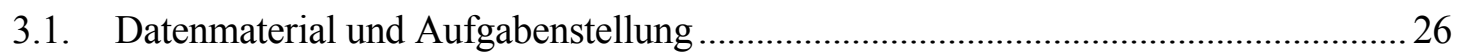

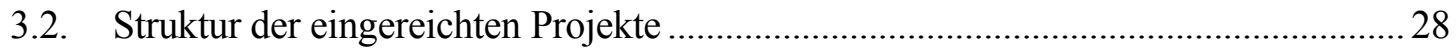

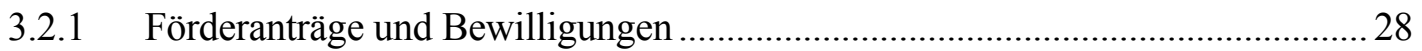

3.2.2 Neue Antragsteller \& Frequenz der Förderanträge............................................. 29

3.2.3 Projektvolumen, Förderbeitrag und Förderbarwert .......................................... 32

3.2.4 Mikrotechnik \& Nanotechnik ........................................................................ 34

3.2.5 Kooperationen in der Initiative Mikrotechnik.................................................... 35

3.2.6 Qualität der Projekte und Patentschutzsstrategien ............................................... 36

3.2.7 Begleitmaßnahmen durch die FFF-Sonderaktionen Feasibility, F\&E Dynamik und Nachwuchsförderungen .............................................................................. 37

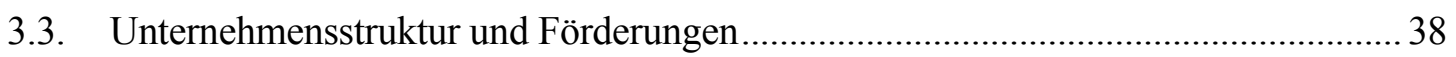

4 RELEVANZ - ODER... „MACHT DER FFF ÜBERHAUPT DAS RICHTIGE?“... 42

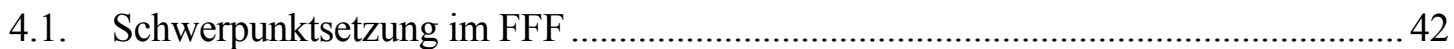

4.2. Mikrotechnik - BASISTECHNOLOGIE _........................................................... 42

4.3. Entspricht die konkrete Zielformulierung der Initiative den Bedürfnissen der

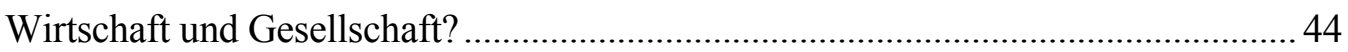

4.4. Wurden die wirtschaftlichen und gesellschaftlichen Rahmenbedingungen

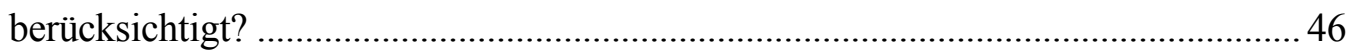

4.5. Mikro \& Nano Förderung im internationalen Kontext .............................................. 47

4.6. Die Initiative Mikrotechnik im Vergleich zur Mikrosystemtechnik 2000+ und zu

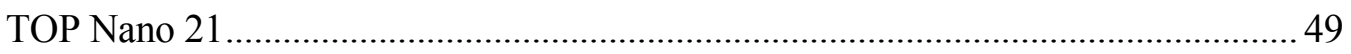

4.7. positionierung der Initiative mikrotechnik zur, Nano Austria' Initiative.....................53

5 INTERNE KOHÄRENZ - ODER... „HAT DER FFF DAS RICHTIGE DESIGN

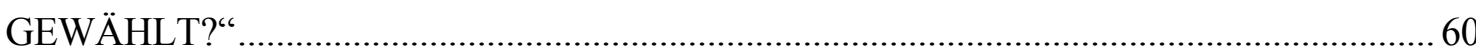

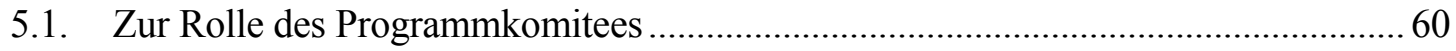

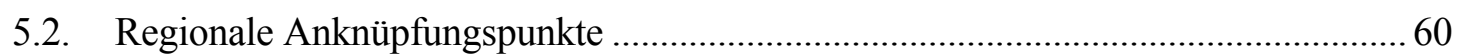

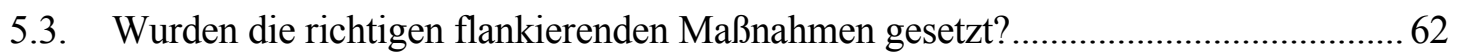

5.4. Sind die gewählten Förderinstrumente dazu geeignet, die selbst gesetzten Ziele zu

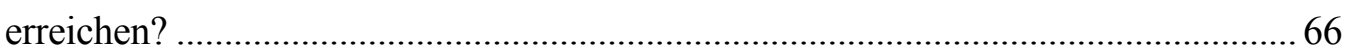

5.5. Welche schlüsse aus der FFF Evaluierung für die Initiative Mikrotechnik gezogen

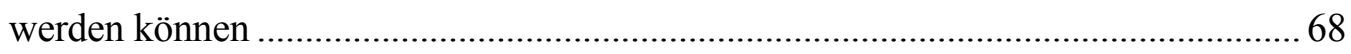

6 EFFEKTIVITÄT - ODER... „WAS HAT DER FFF BEI WEM ERREICHT?“....... 70 
6.1. Was sind die konkreten Outputs der Initiative? ....................................................... 70

6.2. Wie ist der Charakter der geförderten Projekte? ........................................................ 70

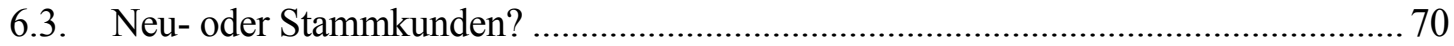

6.4. Grundlagenorientiert oder angewandte Forschung? .................................................. 71

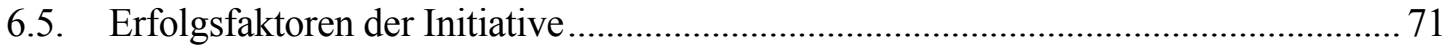

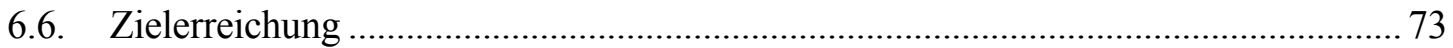

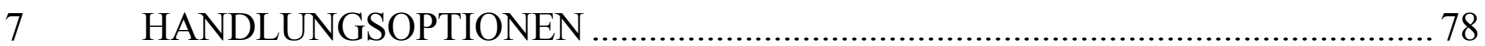

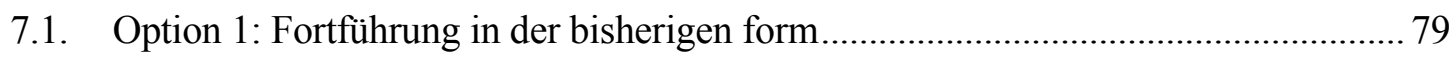

7.2. Option 2: einstellen der Initiative Mikrotechnik ……................................................. 79

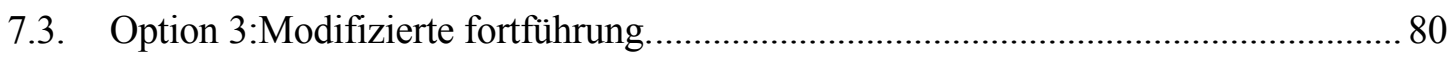

7.4. Option 4: Zusammenführung Mit der NANO Initiative Österreich ............................ 82

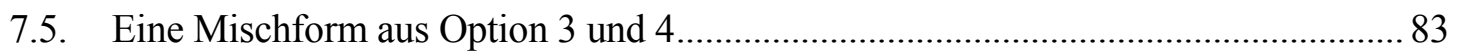

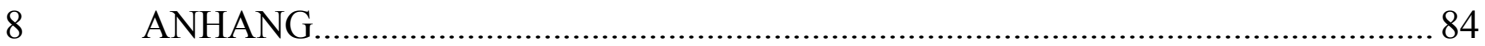




\section{Einleitung}

Mit diesem Bericht legt das Evaluierungsteam der Joanneum Research die Ergebnisse der Zwischenevaluierung der Initiative Mikrotechnik vor. Ziel der Zwischenevaluierung war es, Aufschluss darüber zu geben, ob Adjustierungen bei der „Initiative Mikrotechnik Österreich“ des FFF vorgenommen werden sollten.

Diese Adjustierungen werden nun im Evaluierungsbericht entlang folgender Handlungsoptionen dargestellt werden:

Option 1: Der FFF soll die Initiative „Mikrotechnik Österreich“ so wie bisher fortführen.

Option 2: Der FFF soll die Initiative „Mikrotechnik Österreich“ einstellen.

Option 3: Der FFF soll die Initiative „Mikrotechnik Österreich“ unter Veränderungen des Designs fortführen.

Option 4: Der FFF soll die Initiative „Mikrotechnik Österreich“ mit der Nano Initiatve zusammenzuführen suchen.

Das Evaluierungsteam kommt zum Schluss, dass die Initiative Mikrotechnik in der Form wie bisher nicht weitergeführt werden sollte, sondern einem Redesign unterzogen werden soll.

\section{Inhalte}

Die Zwischenevaluierung der Initiative Mikrotechnik stellt in Kapitel 2 die Initiative vor und identifiziert Mission, Zielsystem und Maßnahmen der Initiative sowie deren operative Zielsetzungen und intendierte Wirkung. Wir bedienen uns der Logic-Chart Analyse, um die Beziehungen zwischen Zielen, Aktivitäten und den verschiedenen Dimensionen der Wirkung der Initiative darzustellen.

In Kapitel 3 werden die Projekte der Initiative Mikrotechnik anhand der Daten zu Projekten und Unternehmen charakterisiert. Als Maß für den Erfolg der Initiative werden unter anderem die quantifizierbaren Erfolgskriterien des Programmkomitees herangezogen. Spezifika der Initiative Mikrotechnik werden mittels Vergleich zur Gesamtheit der FFF-Projekte, die in der Wirkungsanalyse des FFF (im Rahmen der FFF - Evaluierung) erfolgte, ausgearbeitet.

In Kapitel 4 gehen wir der Frage nach, ob eine Schwerpunktsetzung des FFF im Bereich der Mikrotechnologie gerechtfertig ist und ob die konkrete Zielformulierung der Initiative den Bedürfnissen von Wirtschaft und Gesellschaft entspricht. Hierzu wird einerseits der Blick auf die internationale Förderentwicklung gelenkt, andererseits wird auch die Positionierung der Initiative Mikrotechnik gegenüber anderen nationalen Initiativen, Stichwort Nano-Initiative der ASA, beleuchtet.

In Kapitel 5 wird der Frage nachgegangen, ob der FFF das richtige Programmdesign gewählt hat. Wir erörtern die Rolle des Programmkomitees, ermitteln regionale Anknüpfungspunkte, betrachten Anwendung und Nutzung von flankierenden Maßnahmen zur Initiative 
Mikrotechnik und beurteilen ob die gewählten Förderinstrumente geeignet sind, die selbst gesetzten Ziele zu erreichen.

Kapitel 6 fasst schließlich die Wirkungen der Initiative zusammen und beantwortet die Fragen, ob das Technologiefeld Mikrotechnik bzw. Nanotechnik in Österreich durch den FFF weiterentwickelt wurde, ob insbesondere KMU angesprochen wurden, ob zur Kooperation Wissenschaft - Wirtschaft beigetragen wurde, ob regionale Player eingebunden wurden, ob es zu einem internationalen Technologietransfer kam und ob Mitnahmeeffekte bei Unternehmen bzw. Additionalität vorlag.

In Kapitel 7 werden basierend auf den Ergebnissen der Evaluierung Handlungsoptionen für die Zukunft der Initiative Mikrotechnik aufgezeigt: Soll der FFF die Initiative Mikrotechnik wie bisher fortführen, soll die Initiative Mikrotechnik eingestellt werden, soll die Initiative mit Veränderungen fortgeführt werden, oder soll die Initiative mit der Nano-Initiative Österreich zusammengeführt werden?

\section{Methodik}

Neben Dokumentenanalyse, Interviews, einer Logic Chart Analyse und Fallstudien stützen sich Schlüsse und Empfehlungen des Evaluierungsteams im Wesentlichen auf eine Auswertung der vom FFF zur Verfügung gestellten Daten. Wir weisen darauf hin, dass keine Fragebogenerhebung bei den Teilnehmern im Untersuchungsdesign geplant oder gewünscht war.

\section{Zur Definition von Mikrotechnik und Nanotechnologie}

Verschiedene InterviewpartnerInnen mit unterschiedlichem Erfahrungshintergrund haben darauf hingewiesen, dass der Übergang zwischen Mikrotechnik und Nanotechnologie ein fließender und dass die Definitionsarbeit rund um diese Begriffe noch nicht abgeschlossen ist. Entsprechend unserer Kompetenz argumentieren wir in diesem Bericht aus einem forschungsund technologiepolitischen Blickwinkel und beanspruchen nicht, neue und gültige Grenzen zu ziehen.

\section{Mikro- und Nanotechnologie in der Initiative Mikrotechnik}

Wir weisen darauf hin, dass im Rahmen der Initiative Mikrotechnik Projekte der Mikrotechnologie genauso gefördert werden wie Projekte der Nanotechnologie. Das bedeutet für uns, dass, wenn der FFF als Mission seiner Initiative die ,Verankerung und Weiterentwicklung von Mikrotechnik in Österreich' angibt, man richtigerweise ,...von Mikrotechnik und Nanotechnologie' sprechen müsste. Wir ersuchen bei der Lektüre dieses Berichtes diesen Aspekt mitzubedenken.

\section{Initiative, Aktionslinie oder Programm?}

Der FFF spricht im Zusammenhang mit Mikrotechnik immer von einer Initiative oder einer Aktionslinie. Wir verwenden in diesem Bericht die Begriffe „Aktionslinie“ und Initiative synonym. Gegenstand dieser Evaluierung sind des weiteren keinerlei Betrachtungen grundsätzlicher Natur, was nun der Königsweg der österreichischen Technologiepolitik sei: bottom-up Förderung oder Förderung über Programme. Als Orientierungspunkte dienen uns bei dieser Fragestellung allerdings die Ergebnisse der FFF/FWF Evaluierung. 


\section{Ist die Initiative Mikrotechnik mit anderen Sonderaktionen des FFF vergleichbar?}

Die Initiative Mikrotechnik ist, gemeinsam mit der Sonderaktion Holzforschung und der Lebensmitteltechnologieinitiative, die einzige thematische top-down Initiative des FFF. Die EvaluatorInnen möchten jedoch an dieser Stelle davor warnen, diese Initiativen und Sonderaktionen untereinander $\mathrm{zu}$ vergleichen. Gründe hierfür sind die unterschiedliche Laufzeit, der unterschiedliche Technologieintensität der Branchen und die unterschiedlichen Zielsysteme. So war ein Ziel der Sonderaktion Holzforschung, Unternehmen den Einstieg in die Forschungs- und Entwicklungstätigkeit zu ermöglichen. Wir warnen daher, jene Empfehlungen und Schlüsse, die wir aus der gegenständlichen Evaluierung ziehen, unmittelbar auf andere Initiativen umzulegen oder umgekehrt.

\section{FFF und FFG}

Wir geben abschließend zu bedenken, dass in die Laufzeit dieser Evaluierung die Gründung der Forschungsförderungsgesellschaft und somit die Eingliederung des FFF in die FFG fiel. Diese zeitliche Überschneidung ist Grund dafür, dass die Evaluatoren durchgehend noch den FFF, und nicht den FFG-Bereich 2 adressieren. 


\section{JOANNEUM}




\section{Die Initiative „Mikrotechnik Österreich“}

Mission der Initiative „Mikrotechnik in Österreich“ ist es laut Imagefolder, die Entwicklung von mikrotechnischen Produkten und Verfahren in Österreich so $\mathrm{zu}$ fördern, dass sie einen wesentlichen Beitrag zum Ausbau des Standorts Österreichs in zukunftsweisenden Technologien leisten. Die Laufzeit des Programms wurde vom FFF von Jänner 2001 bis Dezember 2003 anberaumt. „Aufgrund des großen Erfolges“, so die Einschätzung des FFF, wurde die Initiative bis Ende 2004 verlängert.

Ursprünglich ging der FFF in Abwesenheit eines fixen Programmbudgets von einem Programmvolumen von 12 Millionen Euro p.a. aus, die Programmrealität hat diesen offensichtlich zu defensiven - Rahmen deutlich überschritten. Insgesamt wurden bisher über 55 Millionen Euro in Projekte der Aktionslinie Mikrotechnik investiert.

Der Fonds fördert im Rahmen des Programms Sach- und Personalkosten in Höhe von bis zu $50 \%$ der gesamten anerkannten Projektkosten. Diese Förderung erfolgt in Form von Darlehen und Zuschüssen oder Haftungen für Darlehen verbunden mit Zinszuschüssen.

Gemäß des Imagefolders zum Programm ging der FFF davon aus, dass ein „,breites Vorgehen“ notwendig sei, um die Mikrotechnik als „Schlüsseltechnologie auf breiter Basis zu verankern“. Hierzu wurden zwei Schienen verfolgt: Einerseits die „klassische F\&E-

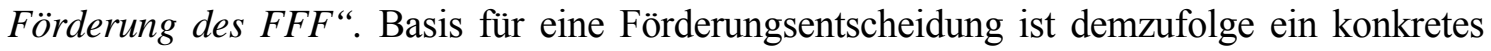
Forschungs- und Entwicklungsprojekt des einreichenden Unternehmens, das

- einen gehobenen Innovationsgrad aufweist,

- ein klar definiertes firmenrelevantes Problem löst und schließlich

- den „Allgemeinen Förderungsrichtlinien“ des FFF entspricht.

Der FFF setzte ,,aufgrund der österreichischen Situation“ einen Schwerpunkt ,im Bereich der Nutzung der Technologie für Anwendungen und Produkte“. Andererseits wurde die Initiative als „Förderung von Technologietransfermaßnahmen“ konzipiert. Also sollten Maßnahmen, die den Einsatz von Mikrotechnik im Sinne der mit F\&E verbundenen Adaptierung folgender Ergebnisse fördern, unterstützt werden:

- Produkt- und Verfahrensverbesserungen,

- Neuentwicklungen,

- Generierung neuer Märkte,

- Findung neuer Anwendungen,

- Verbesserung technischer Prozesse,

- Einsatz neuer Mess- und Prüfverfahren.

Firmen, die in Zusammenarbeit mit nationalen und internationalen Partnern stehen, werden bei der Einführung der neuen Technologien im Rahmen verschiedener Programme, wie z. B. „EURIMUS“ von EUREKA, unterstützt. 
Insgesamt wurden im Projektverlauf ca. 150 Projekte eingereicht, davon wurden 110 für förderwürdig erachtet. Der Anteil der Projekte mit nanotechnologischem Inhalt stieg im Programmverlauf. Im Jahr 2003 waren 12,7\% der Gesamtfördermittel des FFF Projekten der Initiative Mikrotechnik zuzuordnen. Eine quantitative Charakterisierung der Projekte der Initiative erfolgt in Kapitel 3.

Zur Begleitung der Initiative hat der FFF ein Programmkomitee eingerichtet, das das Programm begleitete, den FFF in der Ausrichtung der Initiative beriet und schließlich auch operationalisierte Zielsetzungen (Tabelle 1) entworfen hat.

Tabelle 1: Leitkriterien der Initiative Mikrotechnik - Definition Programmkomitee

\begin{tabular}{l|l}
\hline Leitkriterium & Wertebereich \\
\hline Anzahl der Projekte & $100-150$ \\
Anzahl der antragsstellenden Firmen & $50-70$ \\
Anzahl der Neuantragssteller & $>20 \%$ \\
Kooperationen zwischen Firmen & $>10 \%$ \\
(davon international) & $>30 \%$ \\
Kooperationen mit FH, Hochschule & \\
(davon international) & $>20 \%$ \\
Grenzüberschreitende Kooperationen & $>60 \%$ \\
Anteil der Projekte mit hoher & \\
technologischer Qualität & $30-40 \%$ \\
Projekte mit Patentschutzstrategie &
\end{tabular}

Quelle: Zeilinger, Kukla 2002

Das Formulieren solcher Kriterien war immer wieder eine Forderung an die Akteure der Forschungs- und Technologiepolitik, um Maßnahmen nachvollzieh- und einschätzbarer (evaluierbarer) zu machen und so zu einer besseren, rationaleren Maßnahmenplanung beizutragen. Mit der Formulierung dieser Leitkriterien hat der FFF diesbezüglich eine Vorreiterrolle in Österreich eingenommen.

Der FFF hat nicht nur bottom up Projekte im Rahmen der Initiative Mikrotechnik gefördert, er hat darüber hinaus auch mehrere begleitende Maßnahmen gesetzt: Kooperation mit der Österreichischen Gesellschaft für Mikrosystemtechnik OGMS, um Unternehmen Primärberatung im Technologiefeld zu bieten; es wurde versucht, verschiedene regionale Akteure als Verstärker und Multiplikatoren zu verwenden, die den FFF bei einer „Roadshow“ zur Initiative Mikrotechnik unterstützten. Neben dieser Roadshow wurden zu Beginn des Programms noch verschiedene Awarenessmaßnahmen (zB: eine Podiumsdiskussion zum Thema) vorgenommen.

Wie bei allen Aktionslinien wurde ein Imagefolder produziert, die Homepage der Initiative ist über die wohlstrukturierte FFF-Homepage leicht erreichbar und beinhaltet alle relevanten Informationen.

Als wichtigste Maßnahme neben der Projektförderung ist die Verschränkung mit anderen Aktionslinien des FFF zu sehen: Feasibility Studies (mit dem Ziel externe Machbarkeitsstudien, die von KMUs in Auftrag gegeben werden, durchzuführen), F\&E Dynamik (mit dem Ziel die betriebliche Infrastruktur österreichischer KMUs nachhaltig zu verbessern), Nachwuchsförderung (mit dem Ziel Kooperation und Kommunikation zwischen Wissenschaft und Wirtschaft zu fördern, indem junge ForscherInnen vor dem Studienab- 
schluss gemeinsam mit ihrem/ihrer BetreuerIn und einem Unternehmen ein Forschungsprojekt als Diplomarbeit oder Dissertation realisieren)

Als internationaler Erfolg ist zu werten, dass es dem FFF gelungen ist, die Koordination eines MNT ERA-Nets „From micro- and nano-scale science to new technologies for Europe“ zu übernehmen. Dieses ERA-Nets fördert die Zusammenarbeit nationaler F\&E Initiativen aus dem gegenständlichen Technologiebereich und hat mittel- bis langfristig zum Ziel, auch zu gemeinsamen Ausschreibungen zu kommen.

\subsection{MIKROTECHNIK - LOGIC CHART ANALYSE}

Logic Charts sind Diagramme, die die Beziehungen zwischen Zielen, Aktivitäten, und verschiedenen Dimensionen der Wirkung eines Programms (Outputs, Outcomes, Impact) darstellen. Sie visualisieren das Design, die zugrundeliegenden Annahmen und verbinden es mit gewünschten Effekten. Vorteil von solchen Diagrammen ist die Möglichkeit zur intuitiven Erfassung einer Programmlogik; Lückenanalyse und die Identifikation von (In-)Konsistenzen sind weitere Stärken.

Box 1: Outputs, outcomes, impact

Outputs: the technical results of the projects zB. eine Publikation, ein Prototyp

Outcomes: the direct effects of the project Produktivität des Unternehmens erhöht

Impact: the wider effects of the programme on the society. Lebensqualität erhöht

In principle, outputs cause outcomes, and outcomes cause impacts.

Nach Arnold und Guy: Technology Diffusion Programmes and the Challenge for Evaluation. OECD 1997, Seite 81

Eine Identifikation der allgemeinen, spezifischen und operationalen Zielsetzungen wird durch diese Methode leicht möglich. Die Methode, die ursprünglich aus der Evaluierungsarbeit der US-amerikanischen Kellogg-Foundation ${ }^{1}$ stammt, wurde von Joanneum Research modifiziert, um sie vorrangig für Schritte im Rahmen von Konzept- und Designanalyse einsetzen zu können. Die Anwendung dieser „Logic Chart“ führt zu einem Bild der Initiative Mikrotechnik, das umseitig in Abbildung 2 dargestellt ist. Dieses Bild wurde auf Basis von Dokumentenanalysen und einem Workshop mit dem FFF entwickelt und folgt folgender Struktur:

\footnotetext{
${ }^{1}$ Vgl. hierzu WK Kellogg Foundation Logic Model Development Guide, WK Kellogg Foundation
} 
Abbildung 1: Struktur Logic Chart

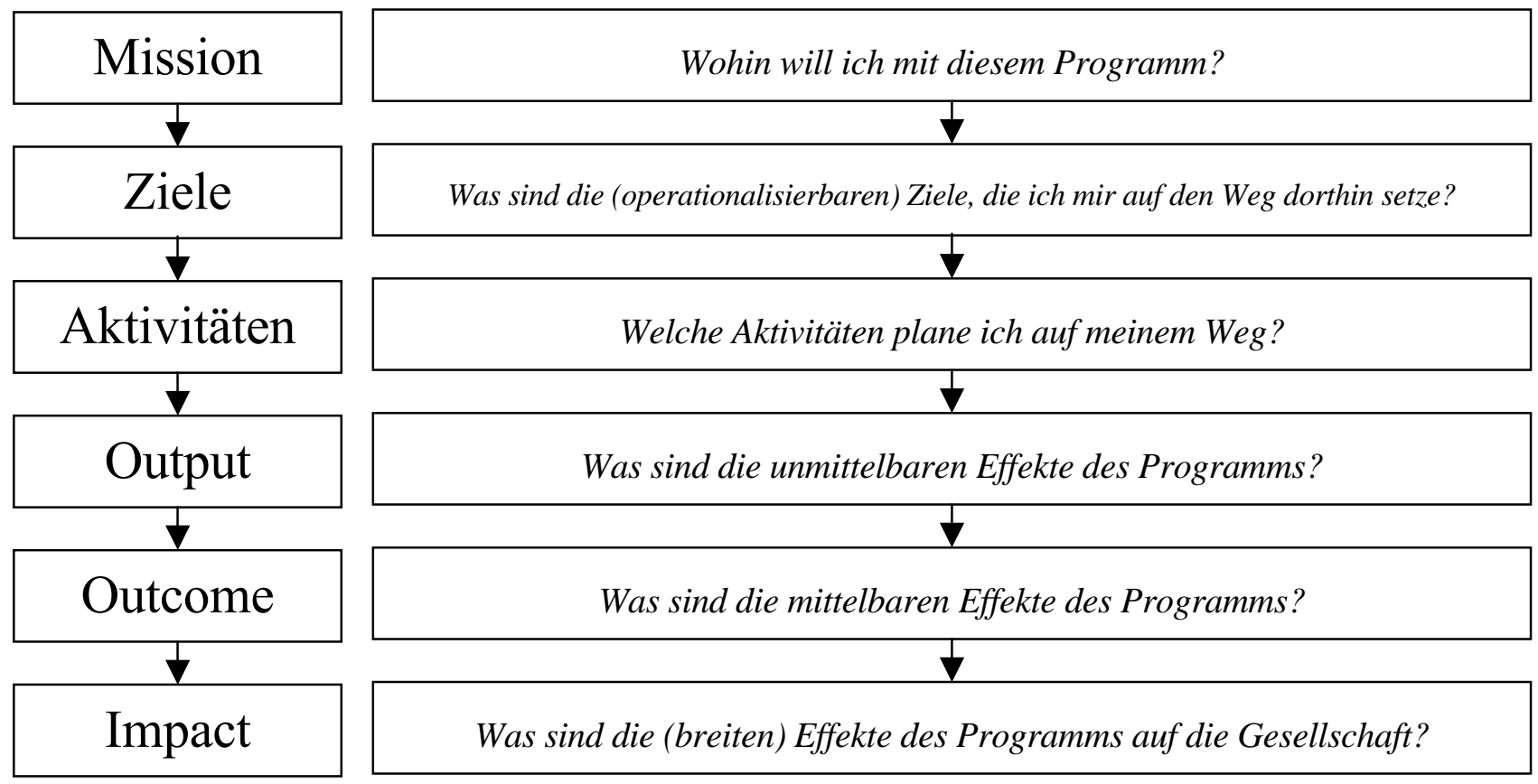

Joanneum Research 
Abbildung 2: Logic Chart der Initiative Mikrotechnik

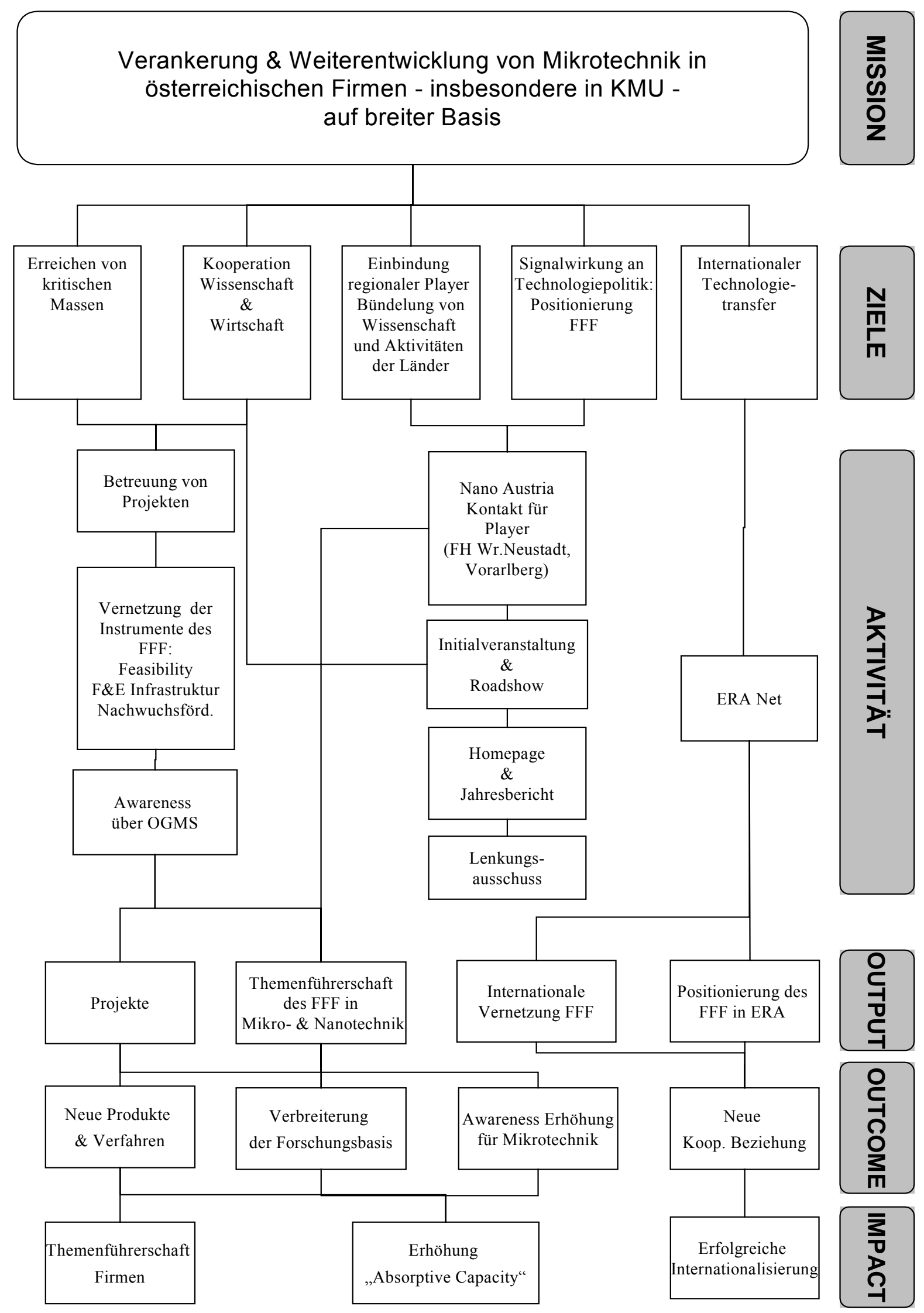




\subsection{MISSION UND ZIELSETZUNG DER INITIATIVE MIKROTECHNIK}

Die Mission der FFF - Initiative Mikrotechnik ist es, Mikrotechnik in Österreich auf breiter Basis zu verankern. Mit Nachdruck wird hier auf kleine und mittlere Unternehmen hingewiesen. Darüber hinaus wird folgender Satz als Schlagzeile verwendet: „Wir wollen vorhandene Technologien zu Firmen bringen und weiterentwickeln.“

Dies lässt für das erste mehrere Stoßrichtungen erkennen:

- „auf breiter Basis“:

o man will also neue Unternehmen bzw.

o neue mikrotechnische Inhalte in Unternehmens F\&E ansprechen

- KMU-Fokussierung

- „vorhandene Technologien zu Firmen bringen“: Technologietransfer

Wie werden diese Stoßrichtungen auf die Zielebene umgesetzt? Ein „Erreichen kritischer Massen“ ist klar mit dem Wunsch verbunden, ,,auf breiter Basis“ Mikrotechnik zu verankern; die Kooperation Wissenschaft - Wirtschaft bzw. die internationale Komponente des Technologietransfers sind offensichtlich mit der Mission verbunden, „vorhandene Technologien zu Firmen zu bringen.“ Die Einbindung der regionalen Player ist aus unserer Sicht ein Ziel, dass als Lukrierung von Vernetzungs- und Multiplikatoreneffekten zu werten ist und sich vernünftig in die Architektur der Initiative einbettet. Der Wunsch, eine Signalwirkung auf die österreichische Technologiepolitik zu haben, kann als Versuch gewertet werden, der Mikrotechnologie in Österreich einen noch höheren Stellenwert einzuräumen.

\subsection{ZU DEN MAßNAHMEN}

Um kritische Massen zu erreichen, bedient sich der FFF seines bedeutendsten Instruments, der Projektförderung. Man hat sich darüber hinaus entschlossen, keine auf die Initiative Mikrotechnik zugeschnittenen Maßnahmen zu setzen (etwa: „Aufbaulehrgang Mikrotechnologie an Universitäten“ $)^{2}$, sondern auf die bestehenden Aktionslinien des FFF zurückzugreifen: Feasibility Studies, F\&E Dynamik. Die gesetzten Awarenessmaßnahmen werden weiter unten eingehend beschrieben, hier kam es zu einer starken Konzentrierung auf eine Kooperation mit der Österreichischen Gesellschaft für Mikrosystemtechnik, OGMS. Die Kooperation Wissenschaft - Wirtschaft wurde über die selbe Maßnahmenpalette umgesetzt, darüber hinaus wurde der Wissenschaft auf der (ebenfalls weiter unten beschriebenen) Roadshow Raum eingeräumt, um selbstständig Kontakte mit der Wirtschaft zu knüpfen.

Einbindung regionaler Player: Vor allem im Rahmen der Roadshow wurde auf eine Zusammenarbeit mit regionalen Akteuren gesetzt; im Lenkungsausschuss des Programms wurde darüber hinaus versucht, wichtige regionale Akteure einzubinden. $\mathrm{Zu}$ engen Kooperationen kam es mit Vorarlberg und der FH Wiener Neustadt; gleichzeitig war man für diese Akteure Ansprechpartner in der Nano Initiative Österreich. Eine Signalwirkung an die österreichische Technologiepolitik abzugeben, wurde ebenfalls über den Lenkungsausschuss, aber auch über Jahresberichte und Homepage versucht. Der Aspekt des „internationalen

\footnotetext{
${ }^{2}$ Dieses Beispiel ist willkürlich gewählt und darf nicht als Vorschlag an den FFF gewertet werden.
} 
Technologietransfers“ wird vorrangig über die Aktivitäten des FFF im ERA Net, dessen Koordinator der Fonds ist, abgedeckt.

\section{Fazit:}

Wir halten das Zielsystem, die gewählten Maßnahmen und die intendierten Wirkungen weitgehenst konsistent. Prima Vista sind kaum Lücken auf Ebene der Maßnahmen erkennbar (auch wenn daraus kein Schluss über die Sinn- und Zweckmäßigkeit der einzelnen Maßnahmen abgeleitet werden kann). Was allerdings der Mission der Initiative besser entsprochen hätte, wäre eine Aktivität gewesen, die klar auf die Beteiligung von KMU an der Initiative abzielen würde.

\subsection{ZU DEN INTENDIERTEN WIRKUNGEN}

Das Papier „Mikrotechnik in Österreich“ von C. Kukla gibt erste Hinweise auf intendierte Outputs, Outcomes, Impacts der Initiative:

\section{Outputs}

- Forschungs- und Entwicklungstätigkeit im Bereich der Mikrotechnik

- Kooperationen zur Nutzung von Synergieeffekten und Bearbeitung komplexer Aufgabenstellungen

- Internationalisierung: Einbindung österreichischer KMUs in internationale, industrielle Netzwerke im Themengebiet Mikrotechnik

\section{Outcomes}

- Entwicklung von mikrotechnischen Produkten und Verfahren in unterschiedlichen Branchen

- Entwicklung von Produkten, die mikrotechnische Komponenten beinhalten

- Stand der Technik in der Mikrotechnik auf breiter Basis anheben

\section{Impacts}

- Auf- und Ausbau Österreichs als Standort zukunftsweisender Technologien

- Erhalt des Wirtschaftsstandortes Österreich

Box 2 stellt nun die in der Logic Chart ermittelten intendierten Wirkungen jenen gegenüber, die Kukla in seinem Motivationspapier identifiziert hat. Hierbei fällt auf, dass der FFF von den ursprünglichen Zielsetzungen des Kukla-Papiers an einigen Stellen abrückt, etwa:

- Die Positionierung des FFF in der österreichischen Technologiepolitikszene findet naturgemäß bei Kukla keine Erwähnung.

- Und schließlich ist der FFF von althergebrachten, blumigen Impact-Vorstellungen wie „Erhalt des Wirtschaftsstandortes Österreich“ abgerückt und hat versucht, Effekte zu intendieren, die eher mit dem Volumen und dem Anspruch einer Initiative wie Mikrotechnik in Verbindung zu bringen sind. 


\begin{tabular}{|c|c|c|}
\hline \multicolumn{3}{|c|}{ Box 2: Gegenüberstellung der intendierten Wirkungen } \\
\hline & Kukla Papier & Logic Chart \\
\hline \multirow{4}{*}{ 营 } & $\begin{array}{l}\text { Forschungs- und Entwicklungstätigkeit } \\
\text { im Bereich der Mikrotechnik }\end{array}$ & Projekte \\
\hline & $\begin{array}{l}\text { Nutzung von Synergieeffekten und } \\
\text { Bearbeitung komplexer } \\
\text { Aufgabenstellungen }\end{array}$ & $\begin{array}{l}\text { Einbindung regionaler Player } \\
\text { - }\end{array}$ \\
\hline & $\begin{array}{l}\text { Internationalisierung: Einbindung } \\
\text { österreichischer KMUs in internationale, } \\
\text { industrielle Netzwerke im Themengebiet } \\
\text { Mikrotechnik }\end{array}$ & $\begin{array}{l}\text { (Internationale Vernetzung FFF) } \\
\text { (Positionierung des FFF in ERA) } \\
\text { - }\end{array}$ \\
\hline & - & $\begin{array}{l}\text { Themenführerschaft des FFF in Mikro- } \\
\text { und Nanotechnik }\end{array}$ \\
\hline \multirow{4}{*}{ 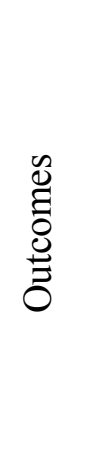 } & $\begin{array}{l}\text { Entwicklung von mikrotechnischen } \\
\text { Produkten und Verfahren in } \\
\text { unterschiedlichen Branchen }\end{array}$ & Neue Produkte und Verfahren \\
\hline & $\begin{array}{l}\text { Entwicklung von Produkten, die } \\
\text { mikrotechnische Komponenten } \\
\text { beinhalten }\end{array}$ & Neue Produkte und Verfahren \\
\hline & $\begin{array}{l}\text { Stand der Technik in der Mikrotechnik } \\
\text { auf breiter Basis anheben }\end{array}$ & $\begin{array}{l}\text { Verbreiterung der Forschungsbasis, } \\
\text { Awareness Erhöhung für Mikrotechnik }\end{array}$ \\
\hline & - & Neue Kooperationsbeziehungen \\
\hline \multirow{3}{*}{ 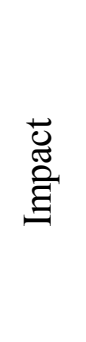 } & $\begin{array}{l}\text { Auf- und Ausbau Österreichs als } \\
\text { Standort zukunftsweisender } \\
\text { Technologien }\end{array}$ & $\begin{array}{l}\text { Themenführerschaft Firmen } \\
\text { Erhöhung der ,Absorptive Capacity,3 } \\
\text { _ }\end{array}$ \\
\hline & $\begin{array}{l}\text { Erhalt des Wirtschaftsstandort } \\
\text { Österreichs }\end{array}$ & - \\
\hline & - & Erfolgreiche Internationalisierung \\
\hline
\end{tabular}

\footnotetext{
${ }^{3}$ Das Konzept der absorptive capacity wurde in mehreren sehr einflussreichen Artikel von W.Cohen und D. Levinthal $(1989,1990)$ entwickelt und sagt im wesentlichen aus, dass F\&E-Tätigkeiten in Unternehmen „zwei Gesichter“ haben. Einerseits dient F\&E der Generierung von neuem Wissen und andererseits erhöht es gleichzeitig die Absorptionsfähigkeit eines Unternehmens, um dadurch extern erzeugtes Wissen nutzen und absorbieren zu können. Absorptive capacity wird definiert als die ,....ability to recognize the value of new information, assimilate it, and apply it to commercial ends" (Cohen, Levinthal 1990).

Cohen, W., D. Levinthal. (1990) Absorptive capacity: A new perspective on learning and innovation. In: Administration Science Quarterly 35(1): 128-152.

Cohen, W., D. Levinthal (1989) Innovation and Learning: The Two Faces of R\&D. in: Economic Journal 99: 569-596
} 


\section{Quantitative Analyse der Mikrotechnik Initiative}

In Kapitel 3 beschreiben wir eingangs das den Evaluatoren zur Verfügung gestellten Datenmaterial und die Aufgabenstellung der quantitativen Analyseschritte. Wir stellen die Struktur der eingereichten Projekte dar, untersuchen die Frequenz der Förderanträge bzw. ob die Antragssteller beim FFF „,neu“ oder „Routinees“ sind.

\subsection{DATENMATERIAL UND AUFGABENSTELLUNG}

Die quantitative Analyse der FFF-Initiative Mikrotechnik für den Zeitraum 2001-2003 erfolgt anhand der vom FFF zur Verfügung gestellten Daten. Der Datenbestand zur Initiative Mikrotechnik enthält einerseits Informationen zu eingereichten Projekten, andererseits Datenmaterial zu den einreichenden Unternehmen.

Die zur Verfügung gestellten Projekt- und die Unternehmensdaten enthalten insbesondere die in Tabelle 2 aufgelisteten für die Projektanalyse der Initiative Mikrotechnik relevanten Kriterien. Unglücklicherweise enthält die Projektdatenbank keine Informationen zu Outputund Verwertungsdaten bzw. Zielen der durchgeführten Projekte, die einen wichtigen Beitrag für eine mögliche Wirkungsanalyse von FFF-Projekten leisten würde. Des weiteren schränkt die Erfassung von Kooperationen mittels Ja/Nein Kriterium die Analysemöglichkeiten ein.

Tabelle 2: Unternehmens- und Projektdaten des FFF

\begin{tabular}{l|l}
\hline Unternehmensdaten & Projektdaten \\
\hline Gesellschaftsform & Unternehmenszuordnung \\
Unternehmensgründung & Projekttitel \\
Unternehmenssitz & Projektlaufzeit \\
Umsatz & Förderzeitraum \\
Exportquote & Projektkosten (Personal, Einrichtung, Sonstige) \\
MitarbeiterInnenanzahl & Fördervolumen \\
F\&E Personal & Förderart (Zuschuss, FFF-Darlehen, Bankkredit) \\
Forschungsintensität & Barwert der Förderung \\
& Wirtschaftskooperation \\
& (ARGE, zu ARGEs kein Unternehmensdaten verfügbar) \\
& Kooperation Wissenschaft-Wirtschaft (Ja/Nein) \\
& Mikrotechnologie versus Nanotechnologie \\
& Wirtschaftliche und technische Projektprüfungsergebnisse \\
\hline
\end{tabular}

Da der FFF Projekte auf einjähriger Basis fördert, und für Projektfortsetzungen neue Förderanträge eingereicht werden müssen, ist zwischen neuen Projekten und Projektfortsetzungen zu unterscheiden.

Projekte der Sonderaktion „Feasibility Studies“ konnten lediglich über Projekttitel identifiziert werden.

Anhand der vorhandenen Daten zu Projekten und Unternehmen erfolgt eine Charakterisierung der Mikro- und Nanotechnologieprojekte vor dem Hintergrund der Zielsetzungen der Initiative entlang der Kriterien Stamm- oder Neukunden des FFF, Unternehmensgröße, Projektgröße und Förderinstrumente.

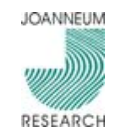


Als klare Erfolgskriterien für die Initiative Mikrotechnik können die in Tabelle 1 angeführten Leitkriterien, die im März 2002 (also nach Beginn der Initiative) vom Programmkomitee erstellt wurden, herangezogen werden. Die darin angeführte untere Grenze der Zielbereiche stellt nach der Ansicht des Programmkomitees eine realistische, die obere eine ambitionierte Grenze dar. Aufgrund der aktuellen Datenerfassung des FFF ist es allerdings nicht möglich, die tatsächlichen Werte für alle Leitkriterien zu erheben.

Im Weiteren wird bei der quantitativen Analyse der Initiative versucht, Spezifika der Projekte und Unternehmen der Initiative im Vergleich zur Gesamtheit der FFF-Förderwerber zu identifizieren. Hierzu wird vor allem auf die Analyse-Ergebnisse der im März 2004 abgeschlossenen Gesamtevaluierung des FFF (Impact Analysis und Internal Functioning and Customer Satisfaction ${ }^{4}$ ) zurückgegriffen.

Fazit:

Der FFF verfügt über ausreichend Datenmaterial um eine Charakterisierung der partizipierenden Unternehmen vorzunehmen, allerdings wäre zusätzlich eine Unternehmenszuordnung nach Branchen mittels NACE Code wünschenswert.

Auf der Projektebene ist eine Verbesserung der Monitoring-Aktivitäten zu empfehlen. Wissenschaft-Wirtschaft Kooperationen werden unzulänglich mittels $\mathrm{Ja} / \mathrm{Nein}$ Kriterium erhoben. Internationale Kooperationen, ein Kriterium der Leitkriterien, können ebenso wenig nachvollzogen werden wie die konkreten Outputs und Vermarktungsmöglichkeiten der Initiative.

Um eine Analyse der intendierten und erzielten Wirkungen der FFF-Projekte zu ermöglichen ist es nötig, zusätzliche Projektdaten elektronisch zu erfassen. Im folgenden werden einige Vorschläge für zukünftige Verbesserungen aufgegriffen:

Kooperationen: Welche Universität, welche Forschungseinrichtung, welches Unternehmen? Hat das Unternehmen bereits vor der Initiative mit Unternehmen oder Universitäten kooperiert? Wenn Ja, mit welchen? War die Kooperation erfolgreich? Werden zukünftige Projekte gemeinsam geplant?

Technik: Werden die Unternehmen im Technologiefeld Mikrotechnik erstmals aktiv, oder trifft die Mikrotechnik ohnehin den operativen Kernbereich des Unternehmens?

Output: Welche Zielsetzungen verfolgt das Projekt? Geht es um die Anwendung von mikrotechnischen Verfahren, oder um Neuentwicklung? Können mit dem Projekt neue Märkte erschlossen werden? Anzahl an beabsichtigten neuen Produkten, Anzahl an angestrebten Patenten und tatsächlich realisierter Output. Zur Nachvollziehung der Effekte auf die geförderten Unternehmen - Stichwort Wirkungsindikatoren (Beschäftigungseffekte, stimulierte Investitionen, Exportsteigerungen etc.), ist eine zusätzliche Verschränkung von Projekt- und Unternehmensdaten nötig.

\footnotetext{
${ }^{4}$ Schibany et al. 2004 bzw. Jörg \& Falk 2004
} 


\subsection{STRUKTUR DER EINGEREICHTEN PROJEKTE}

\subsubsection{Förderanträge und Bewilligungen}

In der Initiative Mikrotechnik 2001-2003 stellten 72 Förderwerber, darunter 7 Arbeitsgemeinschaften (ARGEs), insgesamt 150 Förderanträge von denen 110 (73\%) bewilligt wurden. In Tabelle 3 wird die Verteilung der Förderanträge aufgeschlüsselt nach Unternehmen und Bundesländern dargestellt.

Tabelle 3: Förderanträge nach Bundesländern

\begin{tabular}{l|lllllllll|l}
\hline & Bgld & NÖ & OÖ & Sbg & $\begin{array}{l}\text { Tirol\& } \\
\text { Vbg }\end{array}$ & Wien & Ktn & Stmk & ARGE & Gesamt \\
\hline Unternehmen & 2 & 6 & 7 & 8 & 8 & 10 & 10 & 14 & 7 & 72 \\
Anträge & 3 & 8 & 10 & 12 & 12 & 13 & 21 & 62 & 9 & 150 \\
\hline \multicolumn{2}{l}{ Quelle FFF, eigene Berechnungen }
\end{tabular}

Tabelle 4 stellt die Förderanträge p.a., gegliedert nach neuen Anträgen, Projektfortsetzungen und letztlich wieder zurückgezogenen Projektanträgen dar. Beachtenswert ist der kontinuierliche Anstieg an Förderanträgen um 73\% für neue Projekte von 26 Anträgen im Jahr 2001 auf 45 Anträge im Jahr 2003. Zieht man das Leitkriterium des Programmkomitees zur Beurteilung der Anzahl der Projektanträge heran (100-150 Projekte), so konnten die Erwartungen an die Initiative Mikrotechnik am oberen Ende erfüllt werden. Es muss jedoch berücksichtigt werden, dass in dieser Aufstellung nicht zwischen neuen Projekten und Fortsetzungsprojekten differenziert wird.

Tabelle 4: Förderanträge - Neue Projekte und Fortsetzungen

\begin{tabular}{l|llll}
\hline Projektanträge & 2001 & 2002 & 2003 & Gesamt \\
\hline Neue Projekte eingereicht & 26 & 38 & 45 & 109 \\
davon bewilligt & 20 & 20 & 39 & 79 \\
davon abgelehnt & 6 & 18 & 6 & 30 \\
\hline Fortsetzungen eingereicht & 3 & 15 & 20 & 38 \\
davon bewilligt & 2 & 14 & 15 & 31 \\
davon abgelehnt & 1 & 1 & 5 & 7 \\
\hline Von Unternehmen zurückgezogen & 0 & 2 & 1 & 3 \\
\hline Gesamt & 29 & 55 & 66 & 150 \\
\hline Quelle FFF, eigene Berechnungen & & &
\end{tabular}

Die Rate der Fortsetzungsanträge lag im Zeitraum 2002-2003 bei 29\%. Dies entspricht in etwa der durchschnittlichen Fortsetzungsrate aller FFF-Projekte, die bei 27\% liegt. Dass es bereits im Jahr 2001, zu Beginn der Initiative Mikrotechnik, zu Fortsetzungsprojekten kam ist darauf zurückzuführen, dass der FFF bestehende Projekte mit mikrotechnischem Inhalt der Initiative zugeordnet hat.

Tabelle 5 spiegelt die Ablehnungsraten der Projektanträge wieder. Die durchschnittliche Ablehnungsrate von 27,5\% für neue Projekte und 18,4\% für Projektfortsetzungen weicht nicht erheblich von den üblichen Ablehnungs- bzw. Bewilligungsquoten für FFF Projekte ab (vgl. Schibany et al. 2004). 
Tabelle 5: Ablehnungsraten neue Projekte \& Fortsetzungen der Initative Mikrotechnik in \%

\begin{tabular}{l|llll}
\hline Ablehnungsraten & 2001 & 2002 & 2003 & Gesamt \\
\hline Neue Projekte & 23,1 & 47,4 & 13,3 & 27,5 \\
Fortsetzungen & 33,3 & 6,67 & 25 & 18,4 \\
Gesamt & 24,1 & 35,8 & 16,9 & 25,2 \\
\hline \multicolumn{5}{l}{ Quelle FFF, eigene Berechnungen }
\end{tabular}

Auffallend ist die hohe Ablehnungsquote (47\%) für neu eingereichte Projekte im Jahr 2002, die 9\% über der durchschnittlichen Ablehnungsrate von 38\% für die Gesamtheit der neuen FFF Projekte im Jahr 2002 liegt. Wie in Abbildung $3 \mathrm{zu}$ erkennen, ist diese Ablehnungsrate auf mangelnde Budgetmittel des FFF und nicht auf mangelnde Projektqualität zurückzuführen.

Abbildung 3: Förderanträge und Ablehnungen

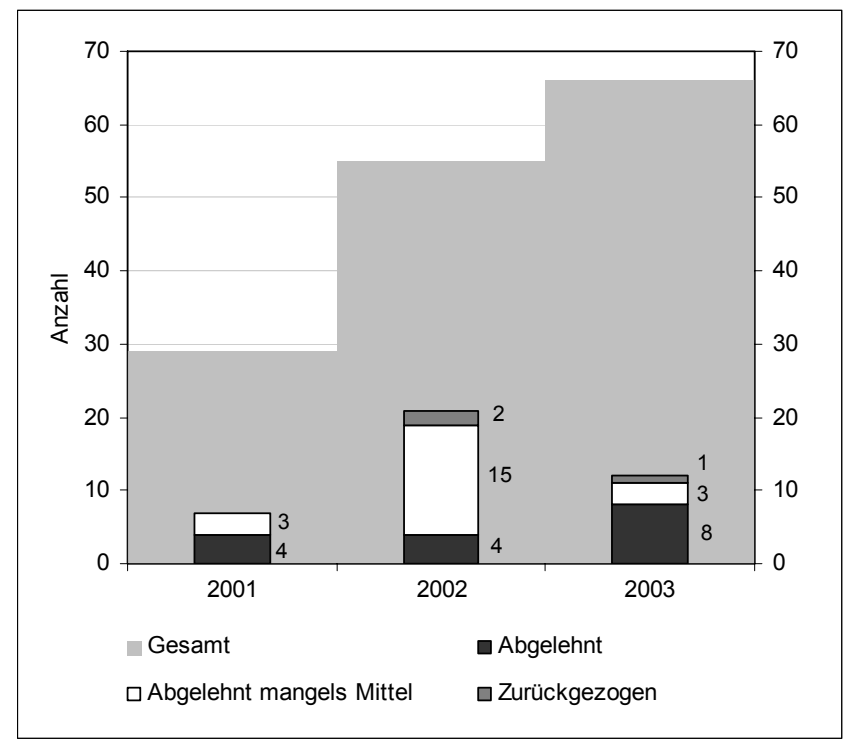

Quelle FFF, eigene Berechnungen

\section{Fazit:}

Das Leitkriterium ,100-150 Projektanträge' konnte unter Berücksichtigung der Fortsetzungsanträge am oberen Ende der Erwartungen erfüllt werden. Die Anzahl der förderwerbenden Unternehmen entsprach ebenso den Erwartungen. Die Bewilligungsrate für Mikrotechnik-Projekte weicht kaum von den üblichen Ablehnungsraten des FFF ab.

\subsubsection{Neue Antragsteller \& Frequenz der Förderanträge}

Der FFF hat Joanneum Research Daten zur Verfügung gestellt, die es nicht erlauben einen direkten Vergleich zu ziehen, welche Unternehmen Neuantragsteller beim FFF sind und welche Unternehmen bereits in der Vergangenheit beim FFF eingereicht haben.

Anhand der zur Verfügung gestellten Daten ist es aber möglich jene Unternehmen zu identifizieren, die an der Initiative Mikrotechnik teilgenommen haben und im Zeitraum 19952004 zusätzliche Projekte eingereicht haben. Unternehmen mit „Fördererfahrung“ können so von Unternehmen, die erstmals eine Förderung in Anspruch nehmen, unterschieden werden. 
In der Initiative Mikrotechnik haben im Zeitraum 2001 - 20039 Unternehmen und 5 ARGEs (die auch aus Unternehmensgruppen bestehen können, die bereits als einzelne Unternehmen beim FFF eingereicht haben) ausschließlich einen Förderantrag für die Initiative Mikrotechnik gestellt. Damit lag die Quote der Antragsteller (exklusive ARGEs) die ausschließlich in der Initiative Mikrotechnik eingereicht haben bei rund 14\%.

Im Durchschnitt hat jeder Antragsteller 2,1 Projktanträge in der Initiative Mikrotechnik gestellt.

Abbildung 4 zeigt die Verteilung der Häufigkeit der Antragstellung. 44 Antragsteller haben ein Projekt, 16 Antragsteller zwei, 6 Antragsteller drei, und 6 Antragsteller 4-20 Projekte eingereicht. Auf jene 6 Unternehmen, die am häufigsten um eine FFF-Förderung in der Initiative Mikrotechnik angesucht haben, entfallen 37\% der Projektanträge und 33\% der durchgeführten Projekte.

Des weiteren zeigt die Abbildung 4 die durchschnittliche Anzahl zusätzlicher Anträge beim FFF in der Periode 1995 - 2004. Die Gesamtheit der Antragsteller der Initiative Mikrotechnik hat sich im Durchschnitt für 18 (Median 5) weitere Projektförderungen beworben.

Jene Unternehmen, die in der Initiative Mikrotechnik die meisten Förderansuchen gestellt haben, sind offensichtlich auch in anderen Forschungsfeldern sehr aktive Förderwerber. Wie in Abbildung $4 \mathrm{zu}$ sehen sinkt in der Initiative Mikrotechnik die Bewilligungsrate erst für Unternehmen mit vier und mehr Förderanträgen ${ }^{5}$.

Abbildung 4: Anträge nach Unternehmen; Mikrotechnik \& insgesamt

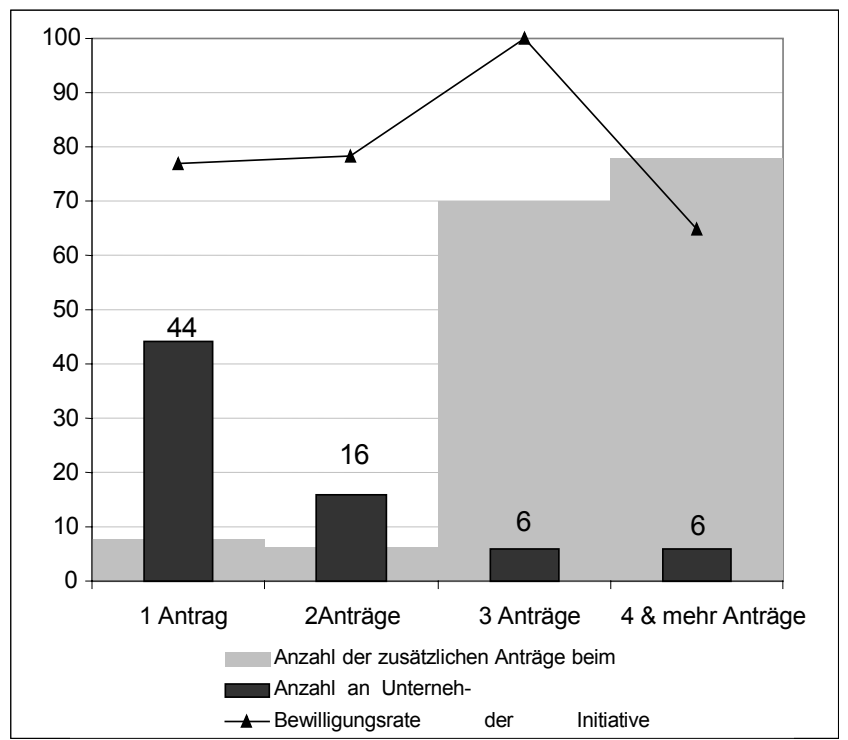

Quelle FFF, eigene Berechnungen

Vergleicht man die Daten der Antragsteller der Initiative Mikrotechnik mit der Gesamtheit der FFF-Förderwerber (siehe Schibany et al. 2004; p. 17) so gewinnt man einen Eindruck über die Aktivität der Förderwerber beim FFF. Laut Wirkungsanalyse hat jedes Unternehmen im Schnitt zwischen 1995 und 2003 2,8 Förderanträge gestellt, während sich die

\footnotetext{
${ }^{5}$ Ein Unternehmen verzeichnete 20 Antragstellungen in der Initiative Mikrotechnik.
} 
Unternehmen der Initiative Mikrotechnik im Durchschnitt für 18 (Median 5) zusätzliche Projekte beworben haben.

Bereits jene 39 Unternehmen (exklusive 5 ARGEs mit nur einem Antrag), die in der Initiative Mikrotechnik lediglich einen Förderantrag stellten, stellten im Durchschnitt 8,4 zusätzliche Förderanträge während 51,7\% (1622 von 3138) von der Gesamtheit der förderwerbenden Unternehmen nur einen einzigen Förderantrag in diesem Zeitraum stellten. Für jene Unternehmen, die 3 und mehr Förderanträge in der Initiative Mikrotechnik stellten, sind die Werte um ein vielfaches höher (vergleiche Abbildung 4 ,zusätzliche Anträge der Mikrotechnik Unternehmen beim FFF“).

Das Heranziehen von Durchschnittswerten verschleiert die große Variabilität der förderwerbenden Unternehmen. In Tabelle 6 befinden sich Durchschnitt, Median, Minimum und Maximum der zusätzlichen Projektanträge, um einen Eindruck von der Streuung der Antragstellung zu vermitteln.

Tabelle 6: Zusätzliche Projektanträge der Mikrotechnik Unternehmen 1995-2003

\begin{tabular}{|c|c|c|c|c|}
\hline & & \multicolumn{3}{|c|}{$\begin{array}{l}\text { Projektanträge in der Initiative } \\
\text { Mikrotechnik }\end{array}$} \\
\hline & & 1 Projekt $^{1}$ & 2 Projekte ${ }^{2}$ & 3 und mehr Projekte ${ }^{3}$ \\
\hline \multirow{4}{*}{ 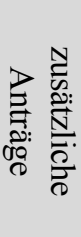 } & Durchschnitt & 8,4 & 7,1 & 74 \\
\hline & Median & 5 & 3 & 45 \\
\hline & Minimum & 0 & 0 & 10 \\
\hline & Maximum & 69 & 30 & 229 \\
\hline
\end{tabular}

Quelle FFF, eigene Berechnungen

\section{Fazit:}

Die Antragsteller der Initiative Mikrotechnik sind deutlich aktivere Förderwerber als die Gesamtheit der Antragsteller. Dies gilt auch unter Berücksichtung der großen Variabilität der förderwerbenden Unternehmen. Die Unternehmen der Initiative Mikrotechnik können daher als Förderroutinees bezeichnet werden.

Zieht man dieses Resultat als Maß für das Erreichen von neuen Zielgruppen heran, so leistet die Initiative nur einen sehr geringen Beitrag zur Verankerung der Basistechnologie Mikrotechnik in Österreich auf breiter Basis, denn die Initiative Mikrotechnik scheint insbesondere die aktivsten Unternehmen anzusprechen und richtet sich in ihrer bisherigen Form nicht an neue Kunden. 


\subsubsection{Projektvolumen, Förderbeitrag und Förderbarwert}

Abbildung 5: Projektvolumen sowie Vergleich „,bewilligt“ - „,abgelehnt“ in tsd. €
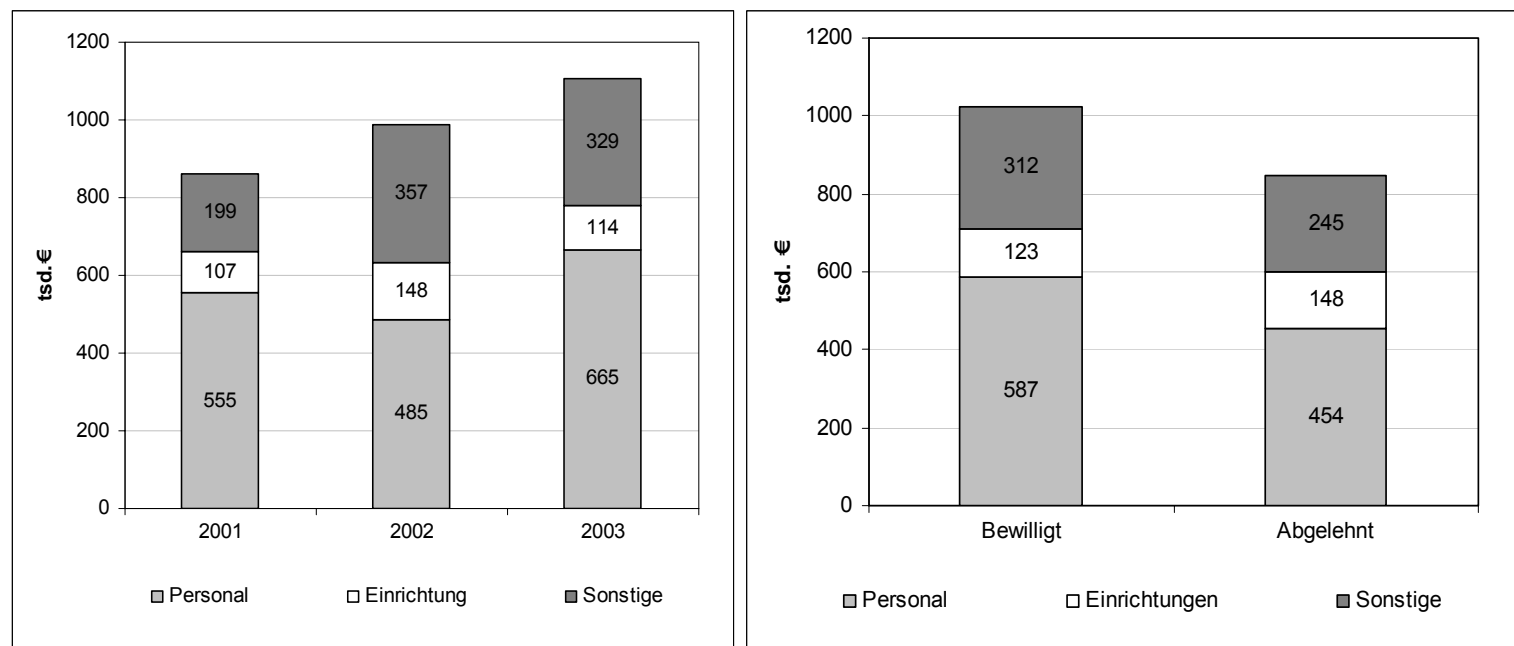

Quelle FFF, eigene Berechnungen

Neben der Anzahl der Förderanträge ist auch das durchschnittliche Projektvolumen der Mikrotechnik Projekte von 862.000€ im Jahr 2001 auf $1.108 .000 €$ im Jahr 2003 angestiegen. Abbildung 5 verfolgt diesen Anstieg unter Berücksichtigung der förderfähigen Projektkosten für Personal, Einrichtung und sonstige Kosten. Die rechte Grafik in Abbildung 5 zeigt, dass die durchschnittlichen Projektkosten der geförderten Projekte höher sind als die der abgelehnten Projekte.

Vergleicht man die Daten zu Projektvolumen und Kostenaufteilung mit den Daten der Gesamtevaluierung des FFF (vgl. Schibany et al. 2004, p. 11), so entspricht die Kostenaufteilung dem Muster der Gesamtheit der FFF-Projekte: Die Personalkosten lagen in den letzten Jahren bei ca. 60\%, die Einrichtungskosten bei ca. 10-15\% und sonstige Kosten bei $20-25 \%$. Im Gegensatz zur Gesamtheit der FFF-Projekte zeichnen sich die Projekte der Initiative Mikrotechnik allerdings durch deutlich höhere Projektkosten aus. So lagen etwa die durchschnittlichen Projektkosten im Jahr 2002 in der Initiative Mikrotechnik bei $989.000 €$ im Gegensatz zu $600.000 €$ für die Gesamtheit der FFF-Projekte.

Abbildung 6: Durchschnittlicher Förderbeitrag (tsd. €) und Funding Mix der Initiative
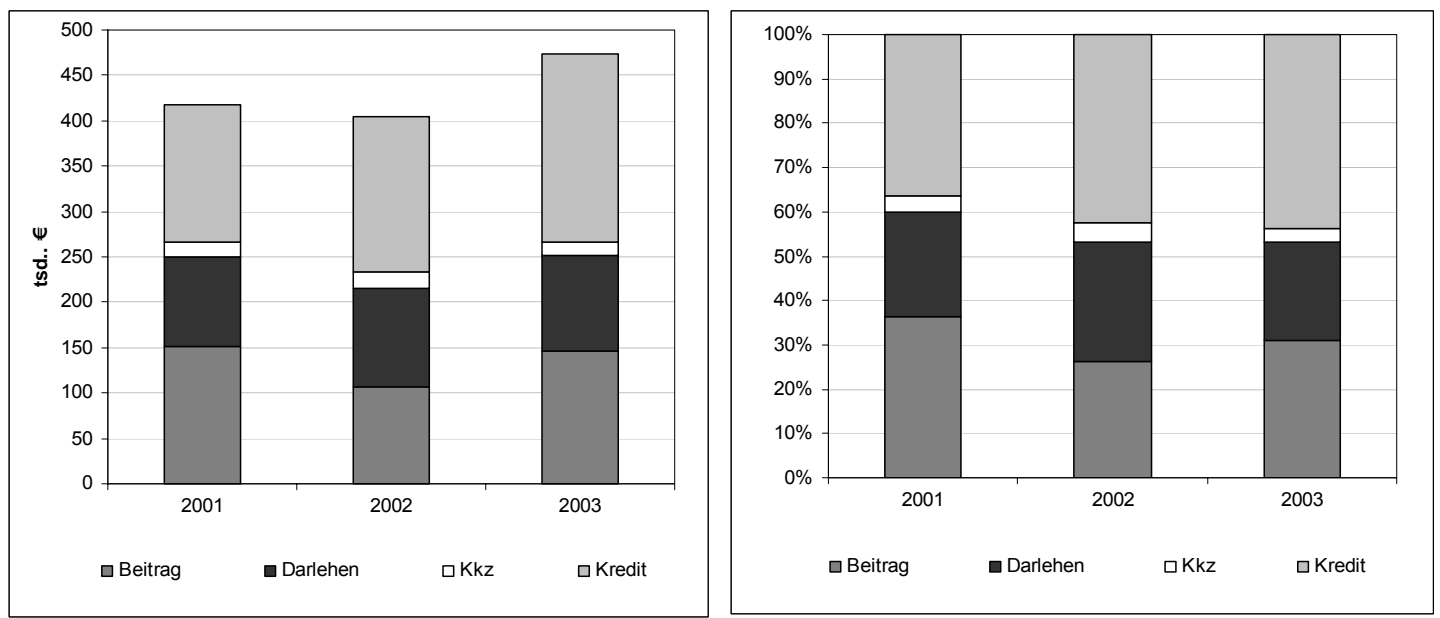

Quelle FFF, eigene Berechnungen 
Der durchschnittliche Förderanteil je Projekt liegt in der Initiative Mikrotechnik bei $442.000 €$ oder 43,2 \% der Projektkosten. Da die FFF-Förderung allerdings aus einem Fördermix aus Beiträgen, Darlehen, Kreditkostenzuschüssen, Bankkrediten sowie EU-Beiträgen darstellt, ist der Barwert der Förderung geringer als der FFF-Förderbeitrag. Der Barwert der Förderung entspricht durchschnittlich 22,1\% oder 226.000 $€$ der Projektkosten. Damit liegen sowohl Förderanteil als auch Förderbarwert im üblichen Bereich des FFF-Fördermixes, die um 45\% Förderhöhe und 20-23\% Förderbarwert je Projekt pendelt. Aufgrund der ähnlichen Ausprägung von Förderbarwert und Fördermittel je Projekt beanspruchen die Projekte der Initiative Mikrotechnik mehr Fördermittel als der Durchschnitt der FFF-Projekte.

Tabelle 7: Gesamtfördermittel nach Budgetjahren und Barwert der Förderungen

\begin{tabular}{l|lll}
\hline Budgetjahr & $\begin{array}{l}\text { Bewilligte } \\
\text { Projekte }\end{array}$ & $\begin{array}{l}\text { Fördermittel } \\
\text { in Mio } €\end{array}$ & $\begin{array}{l}\text { Förderbarwert in } \\
\text { Mio } €\end{array}$ \\
\hline 2001 & 22 & 9,34 & 4,2 \\
2002 & 34 & 16,80 & 7,8 \\
2003 & 54 & 28,91 & 12,9 \\
Insgesamt & 110,0 & 55,05 & 24,9 \\
\hline
\end{tabular}

Quelle FFF, eigene Berechnungen

Der für die Initiative Mikrotechnik vorgesehene Budgetrahmen liegt bei 12 Mio $€$ pro Jahr. Während im Jahr 2001 die Fördermittel noch nicht im vollen Ausmaß ausgeschöpft wurden, wurden die Fördermittel im Jahr 2002 um rund 4,8 Mio € und im Jahr 2003 sogar um mehr als das doppelte überschritten. Nimmt man hingegen den Barwert der Förderung als Maß für das Förderbudget, so wurde der vorgesehene Budgetrahmen erstmals im Jahr 2003 voll ausgeschöpft.

Abbildung 7: Bewilligungsrate und Förderbarwert nach Projektgröße

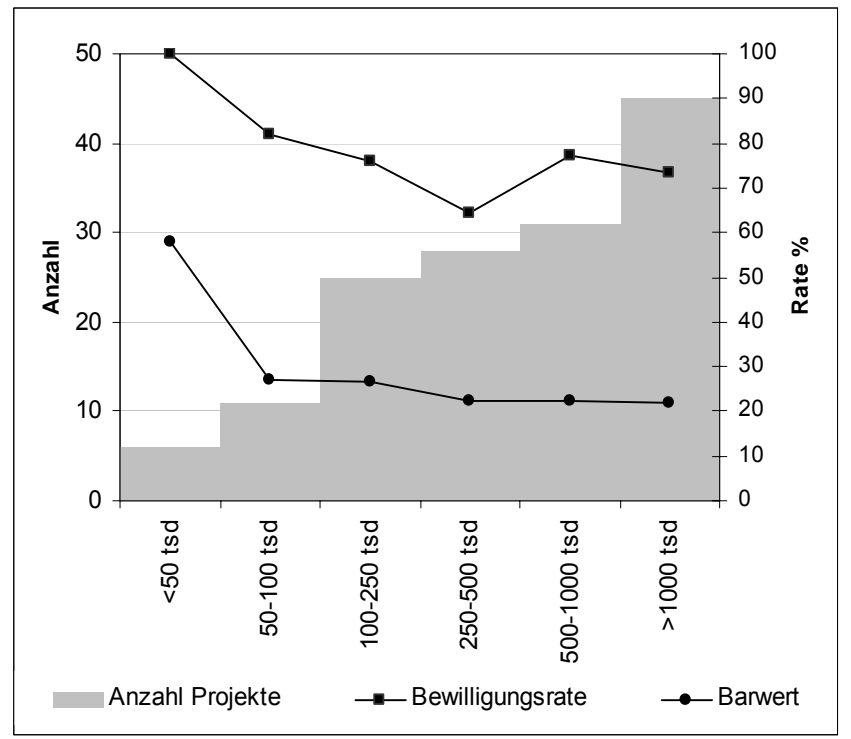

Quelle FFF, eigene Berechnungen

Abbildung 7 zeigt die Verteilung der 150 Projektanträge auf monetäre Größenklassen mit korrespondierenden Bewilligungs- und Förderbarwertraten. Die Mehrzahl der Förderanträge befindet sich in den Gruppen der Großprojekte mit mehr als $500.000 €$ Gesamtprojektvolumen; Eine Verteilung die klar von der Verteilung der Förderprojekte in der Wirkungsanalyse der FFF-Projekte von Schibany et al. (p. 13) abweicht. 
Die Verteilung der Projekte auf Größenklassen bietet neben dem höheren durchschnittlichen Projektvolumen einen weiteren klaren Hinweis dafür, dass Projekte in der Mikro- und Nanotechnologie finanzmittelintensiver als in anderen Forschungsfeldern sind.

Schibany et al. (p. 13) identifizieren in ihrer Wirkungsanalyse des FFF eine Korrelation zwischen Projektgröße und Förderhöhe, derzufolge die Bewilligungsrate mit steigender Projektgröße zunimmt, während die Barwertförderquote mit steigender Projektgröße abnimmt.

Diese Entwicklung kann für die Subgruppe der Mikrotechnik-Projekte nicht nachvollzogen werden (siehe Abbildung 7). In der Initiative Mikrotechnik sinkt die Bewilligungsquote bis zu einer Projektgröße von $500.000 €$, um dann erst wieder anzusteigen. Der Förderbarwert sinkt, abgesehen von der ersten Gruppe der Projekte, welche 3 kleine Feasibility Studies zu ca. $10.000 €$ beinhaltet, nur marginal.

\section{Fazit:}

Die Projekte der Initiative Mikrotechnik zeichnen sich durch deutlich höhere Projektkosten als die Gesamtheit der FFF-Projekte aus. Die Mehrzahl der Förderprojekte verzeichnet Projektkosten von über $500.000 €$. Der Budgetrahmen von 12 Mio € pro Jahr, gemessen an den gesamt bewilligten Fördermitteln, wurde im Jahr 2002 leicht und im Jahr 2003 beinahe um das doppelte überschritten.

\subsubsection{Mikrotechnik \& Nanotechnik}

Abbildung 8: Mikrotechnologie und Nanotechnologie

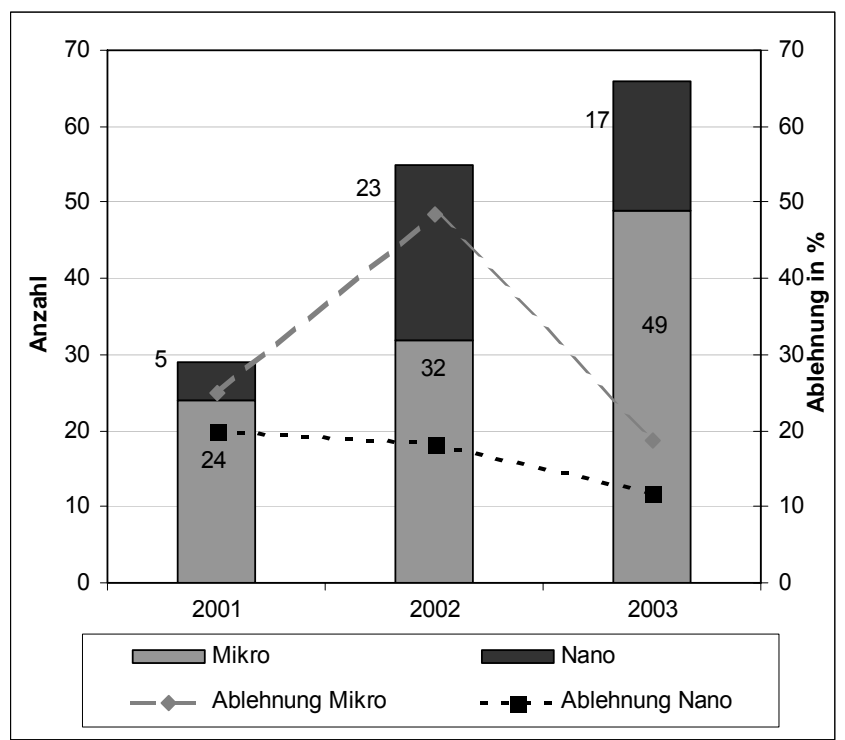

Quelle FFF, eigene Berechnungen

Die Initiative Mikrotechnik beinhaltet Projekte mit mikro- und nanotechnischen Forschungsschwerpunkten. Die Zuordnung der Projekte zur Mikro- bzw. Nanotechnologie erfolgt durch die technischen Prüfer des FFF. Von den 150 eingereichten Projekten wurden 105 der Mikrotechnologie und 45 der Nanotechnologie zugeordnet. Dies entspricht einem Verhältnis von 70:30. 
Abbildung 8 zeigt die jährliche Einreichung von Mikro- und Nanotechnik Projekten mit den entsprechenden Ablehnungsraten. $\mathrm{Zu}$ sehen ist, dass die Ablehnungsrate für NanotechProjekte unter der Ablehnungsrate von Mikrotechnik Projekten liegt.

Tabelle 8 enthält zusätzlich zu Projekteinreichungen und Bewilligungen die Fördersumme der bewilligten Projekte aufgeschlüsselt nach den Technologien Mikro- und Nano.

Tabelle 8: Mikro- und Nanotechnologie in Fördergeldern

\begin{tabular}{l|lllll}
\hline & Positiv & \multicolumn{4}{l}{ Davon Fördersumme } \\
& bewertet & Nano in Mio Eur & $\begin{array}{l}\text { Davon } \\
\text { Mikro }\end{array}$ & $\begin{array}{l}\text { Davon } \\
\text { Nano }\end{array}$ \\
\hline 2001 & 22 & 4 & 9,34 & 8,42 & 0,92 \\
2002 & 34 & 18 & 16,80 & 8,52 & 8,28 \\
2003 & 54 & 15 & 28,91 & 18,21 & 10,7 \\
In Summe & 110 & 37 & 55,05 & 35,15 & 19,9 \\
\hline
\end{tabular}

Quelle FFF, eigene Berechnungen

\section{Fazit:}

Mikro- und Nanotechnologie Projekte stehen einander in der Initiative als ebenbürtig förderfähig gegenüber. Der Anteil an Mikrotechnik Projekten ist mit 70\% noch deutlich höher als der Anteil an Nanotechnik Projekten, Nano-Projekte weisen jedoch einen positiven Trend auf.

\subsubsection{Kooperationen in der Initiative Mikrotechnik}

Von den 150 Projektanträgen der Initiative Mikrotechnik wurden 114 (76\%) ohne Wissenschaft-Wirtschaft Kooperation , 36 Projekte (24\%) wurden in Kooperation mit Forschungseinrichtungen eingereicht. 9 Projekte wurden von 7 Arbeitsgemeinschaften (ARGE) zwischen Unternehmen eingereicht. Damit konnte das Leitkriterium für Kooperationen zwischen Unternehmen $(>10 \%$ ) nicht erfüllt werden. Für internationale Kooperationen zwischen Unternehmen existieren keine Informationen in den vom FFF übermittelten Daten.

Abbildung 9: Wissenschaft-Wirtschaft Kooperationen nach Projekt- und Unternehmensgröße
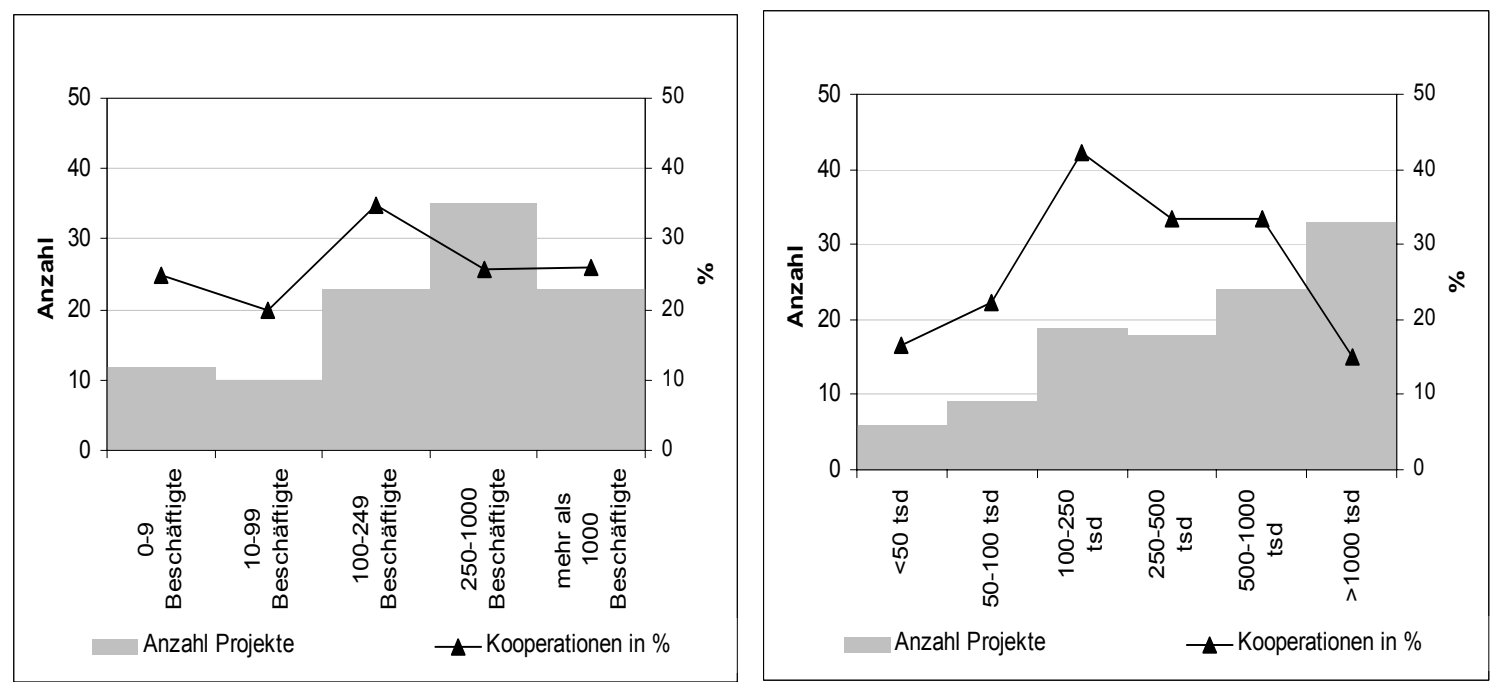

Quelle FFF, eigene Berechnungen 
Von den 110 bewilligten Projekten werden rund 73\% ohne Wissenschaft - Wirtschaft Kooperation durchgeführt und 27\% mit Kooperationen. Damit konnte der in den Leitkriterien angeführte Wert von 30\% knapp nicht erreicht werden. Die Ablehnungsquoten für Projekte mit Wissenschaft-Wirtschaft Kooperationen unterscheiden sich nicht wesentlich von den Ablehnungsquoten ohne Wissenschaft-Wirtschaft Kooperationen.

Abbildung 9 schlüsselt die bewilligten Projekte der FFF Initiative Mikrotechnik nach Beschäftigten-Anzahl und Projektgröße auf, um ein erweitertes Bild davon zu schaffen, welche Projekte mit und welche ohne Wissenschaft - Wirtschaft Kooperation durchgeführt werden. Die Gruppe der mittelgroßen Unternehmen mit 100 bis 250 Beschäftigten, die für rund $21 \%$ der durchgeführten Projekte verantwortlich zeichnet, kooperiert am aktivsten mit Forschungseinrichtungen und Universitäten. Des weiteren ist der Anteil an Kooperationsprojekten von größeren Unternehmen mit rund 26\% noch etwas größer als jener von kleinen Unternehmen, wobei aufgrund der geringen Anzahl an Projekten von Kleinunternehmen keine Verallgemeinerung getroffen werden darf.

Ein ähnliches Bild ergibt sich, wenn die Projekte der Initiative Mikrotechnik nach der Projektgröße aufgeschlüsselt werden. In der Klasse der Projekte mit einem Projektvolumen von $100.000-250.000 €$ haben wird in mehr als 40\% der Fälle mit Forschungseinrichtungen oder Universitäten kooperiert, während der Anteil an Kooperationsprojekten mit einer Projektgröße von mehr als 1 Mio $€$ auf $15 \%$ fällt.

\section{Fazit:}

Der in den Leitkriterien angepeilte Wert von 30\% Wissenschaft-Wirtschaft Kooperationen konnte knapp nicht erreicht werden. Vor allem innovative mittelständische Unternehmen die über nicht genügen ,in house“ Forschungskapazitäten verfügen, scheinen auf externe Forschungskapazitäten zurückzugreifen. Der FFF setzt zwar Anreize für Kooperationen (etwa über einen höheren Förderbarwert), stellt das nach außen hin jedoch nicht genügend dar.

Die Quote der Unternehmenskooperationen blieb hinter den Erwartungen des Programmkomitees zurück. Der Anteil an internationalen Kooperationen wurde nicht erhoben.

Auf Grund der nur unbedeutend unterschiedlichen Ablehnungsrate zwischen Projekten „mit“ und „ohne“ Kooperationen kann keine Aussage getroffen werden, ob die Initiative Mikrotechnik einen zusätzlichen Kooperationsimpuls geleistet hat.

\subsubsection{Qualität der Projekte und Patentschutzsstrategien}

Die interne Projektbewertung mittels eines formalisierten, EDV gestützten Systems nach wissenschaftlichen und wirtschaftlichen Kriterien auf der Projekt- und Unternehmensebene stellt eine Hauptaktivität des FFF dar. Für die Analyse der Projekte in der Initiative Mikrotechnik gelten die selben Maßstäbe wie für alle übrigen Projekte. Da der Prozess der Projektbewertung bereits in der Gesamtevaluierung des FFF einer Beurteilung unterzogen wurde, verweisen wir in diesem Zusammenhang auf die Ergebnisse dieser Evaluierung (Synthesis Report - Evaluation of the Austrian Industrial Research Promotion Fund (FFF) and the Austrian Science Fund (FWF), 2004). 
In den Leitkriterien des Programmkomitees wurden Zielwerte für den Anteil der Projekte mit hoher technologischer Qualität $(>60 \%)$ und für den Anteil der Projekte mit Patentschutzstrategie (30\% - 40\%) festgelegt. Für eine Analyse der Bewertung der Qualität des Projektportfolios wurden keine eigenen Berechnungen durchgeführt, sondern auf die internen Auswertungen der Projektbewertung des FFF zurückgegriffen.

Hinsichtlich Projekte mit Patentschutzstrategie haben laut FFF 48\% der Projekte konkrete Überlegungen gemacht, oder Schritte zur Erlangung eines Patents gesetzt. Zusätzlich ist bei rund 13\% der Antragsteller ein Patent vorhanden, eingereicht oder erteilt worden. Damit konnte der Zielbereich von 30-40\% klar übertroffen werden.

Was den Anteil der Projekte mit hoher technologischer Qualität betrifft, so wurde mit einem Zielbereich von mehr als 60\% zwar ein Kriterium aufgestellt, jedoch kein Schwellenwert definiert, der Projekte mit technologischer Qualität auszeichnet.

Zur Bestimmung der technologischen Qualität wurden jene 76 Projekte herangezogen, von denen schon ein Endbericht vorhanden ist (Stichtag 1.9.2004) und welche bereits von einem Techniker bewertet wurden. Bei einer maximal zu Punktezahl von 100 (Kombination von P und T-Wert), ergibt sich folgende Verteilung:

Tabelle 9: Technologische Qualität der Projekte

\begin{tabular}{c|l}
\hline Punkte & Verteilung der Projekte \\
\hline$<70$ & sind 28 Projekte (37\%) \\
$70-74$ & sind 20 Projekte (26\%) \\
$75-79$ & sind 11 Projekte (15\%) \\
$80-89$ & sind 16 Projekte (21\%) \\
$90-100$ & ist 1 Projekt (1 \%) \\
\hline Quelle FFF, eigene Berechnungen
\end{tabular}

Definiert man den Schwellenwert für „technologische Qualität“ etwa $20 \%$ über dem Schwellenwert für die ex-ante Förderung, so können $63 \%$ der Projekte als technologisch hochwertig betrachtet werden, definiert man den Schwellenwert $25 \%$ über dem Schwellenwert der ex-ante Förderung so können $37 \%$ der Projekte als technologisch hochwertig betrachtet werden.

\subsubsection{Begleitmaßnahmen durch die FFF-Sonderaktionen Feasibility, F\&E Dynamik und Nachwuchsförderungen}

Im Programmfolder des FFF zur Initiative Mikrotechnik und in der Logic-Chart Analyse wird insbesondere auf die Zielsetzung der Vernetzung der Initiative Mikrotechnik mit den FFFSonderaktionen Feasibility, F\&E Dynamik und Nachwuchsförderung zur Erreichung von kritischen Massen hingewiesen. Der Datenbestand zur Initiative Mikrotechnik erlaubt die Identifikation der Projekte zur Sonderaktion Feasibility und zur Sonderaktion F\&E Dynamik im Zusammenhang mit der Mikrotechnik.

In den 150 Förderantragen befinden sich 2 Feasibility-Studies im Bereich der MikroTechnologie und eine Feasibility Study im Bereich Nano-Technologie. Alle 3 Projekte mit 
Gesamtkosten von je $10.000 €$ wurden bewilligt und mit einem Beitrag von 70\% gefördert. Insgesamt wurden im Beobachtungszeitraum 2001-2003 158 Feasibility Studies durchgeführt.

In der Sonderaktion F\&E Dynamik wurden von 2001-2003 36 Projekte durchgeführt. Von diesen 36 Projekten konnte keines der Initiative Mikrotechnik zugeordnet werden, ein Unternehmen der Initiative Mikrotechnik nahm an der Sonderaktion F\&E Dynamik teil, jedoch mit einem Projekt ohne mikro- oder nanotechnischem Forschungsschwerpunkt.

\section{Fazit:}

Im Programmfolder des FFF wurde insbesondere auf die Vernetzung der verschiedenen Förderinstrumente bzw. Aktionslinien des FFF aufmerksam gemacht. Insbesondere die Aktionslinien Feasibility und F\&E Dynamik stellen gute Möglichkeiten dar um kleinen und mittleren Unternehmen den Zugang zu Anwendungs- und Forschungsmöglichkeiten im Bereich der Mikrotechnik zu ermöglichen.

Die Unternehmen nutzten die vorgeschlagenen Begleitmaßnahmen zur Initiative Mikrotechnik nur sehr eingeschränkt. Im gesamten Förderzeitraum der Initiative Mikrotechnik wurden im Rahmen der Mikrotechnik Initiative 3 Feasibility Studies von insgesamt 158 Feasibilty Studies beantragt. Von den 36 F\&E Dynamik Projekten im Beobachtungszeitraum 2001-2003 wurde keines der Initiative Mikrotechnik zugerechnet. Die Zielsetzung der Vernetzung der Förderinstrumente des FFF konnte nicht erreicht werden.

\subsection{UNTERNEHMENSSTRUKTUR UND FÖRDERUNGEN}

In der Logic-Chart Analyse wurde die Mission der Mikrotechnik wie folgt umrissen: „Verankerung und Weiterentwicklung von Mikrotechnik in Österreich auf breiter Basis, insbesondere in Klein- und Mittelunternehmen“. Als Schlagzeile: „Wir wollen die vorhandene Technologie zu Firmen zu bringen und weiter entwickeln“. Von dieser Mission abgeleitet existiert die Zielsetzung des Programms ,kritische Massen“ zu erreichen.

Im Kontext einer Charakterisierung der FFF-Initiative Mikrotechnik anhand der eingereichten Struktur der Projekte kann das Ziel „kritische Massen“ erzeugen zu wollen, dahingehend interpretiert werden, dass mehr und neue Unternehmen im Bereich der Mikrotechnologie forschen bzw. das mehr und neue Unternehmen mikrotechnische Verfahren und Produkte anwenden -sprich in ihre Produkte mittels Anwendungsforschung implementieren.

Im Kapitel 3.2.1 „Förderanträge und Bewilligungen“ wurde bereits auf die Zahl der Förderwerber eingegangen und im Kapitel 3.2.2 „Neue Antragsteller \& Frequenz der Förderanträge" wurde die Anzahl der neuen Antragsteller beim FFF analysiert.

Ein weiterer Aspekt, der das Erreichen von kritischen Massen anspricht, betrifft die Verankerung der Mikrotechnik in Österreich insbesondere in KMUs. Vor diesem Hintergrund erfolgt eine Analyse der Mikrotechnik Projekte mit speziellem Blickwinkel auf die antragstellenden Unternehmen.

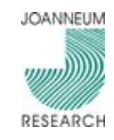


Abbildung 10: Unternehmensverteilung nach Anzahl der Beschäftigten und Förderanträge

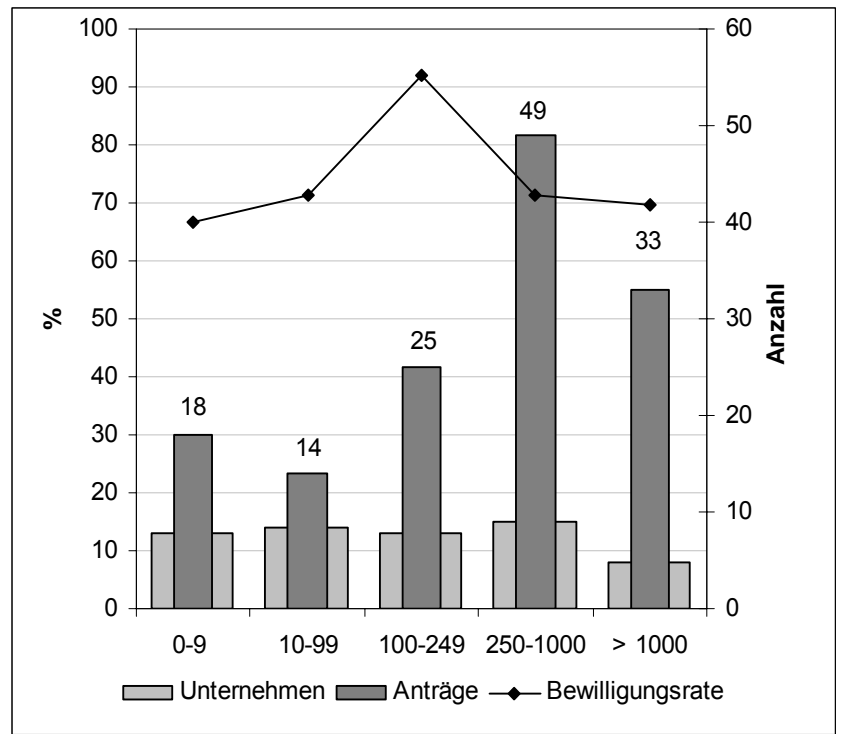

Quelle FFF, eigene Berechnungen

Von den 63 Unternehmen (exklusive ARGEs) die Förderanträge in der Initiative Mikrotechnik gestellt haben, können 40 Unternehmen oder $63 \%$ aufgrund ihrer MitarbeiterInnenanzahl von weniger als 250 der Gruppe der kleinen und mittleren Unternehmen zugerechnet werden. Die Bewilligungsquote ist für die Gruppe der mittelgroßen Unternehmen mit 100-249 Beschäftigten am höchsten, die Quote für große Unternehmen ist mit jenen der kleinen Unternehmen vergleichbar.

Zieht man die Anzahl der Projektanträge in der Initiative Mikrotechnik als Maß für die Einbindung der kleinen und mittleren Unternehmen an der Initiative Mikrotechnik heran, so sinkt die Beteiligung der KMUs an der Initiative auf 41\%. Der starke Zusammenhang zwischen Förderanträgen und MitarbeiterInnenanzahl wird in Abbildung 11 deutlich.

Abbildung 11: Förderanträge und Unternehmensgröße

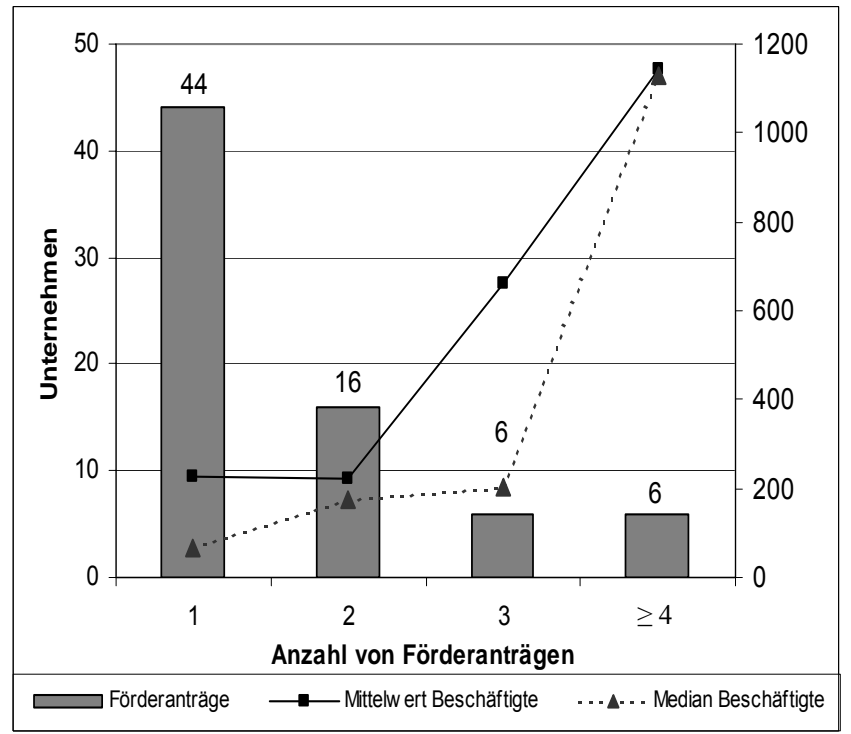

Quelle FFF, eigene Berechnungen 
Jene 6 Unternehmen, die mehr als 3 Förderanträge in der Initiative stellten, sind ausschließlich große Unternehmen mit durchschnittlich mehr als 1000 Beschäftigten. Im Detail betrachtet, zeichnen diese Unternehmen, die sich am häufigsten für FFF-Projekte in der Initiative Mikrotechnik beworben haben, für insgesamt 56 oder 37,7\% der 150 Förderanträge verantwortlich. Auf sie entfallen rund ein Drittel der bewilligten Förderanträge. In Fördermitteln ausgedrückt lukrieren diese 6 aktivsten Antragsteller 46,7 \% der gesamten Fördermittel und 46,4\% des gesamten Förderbarwertes.

Abbildung 12: Fördermix und Fördermittel nach Unternehmensgröße

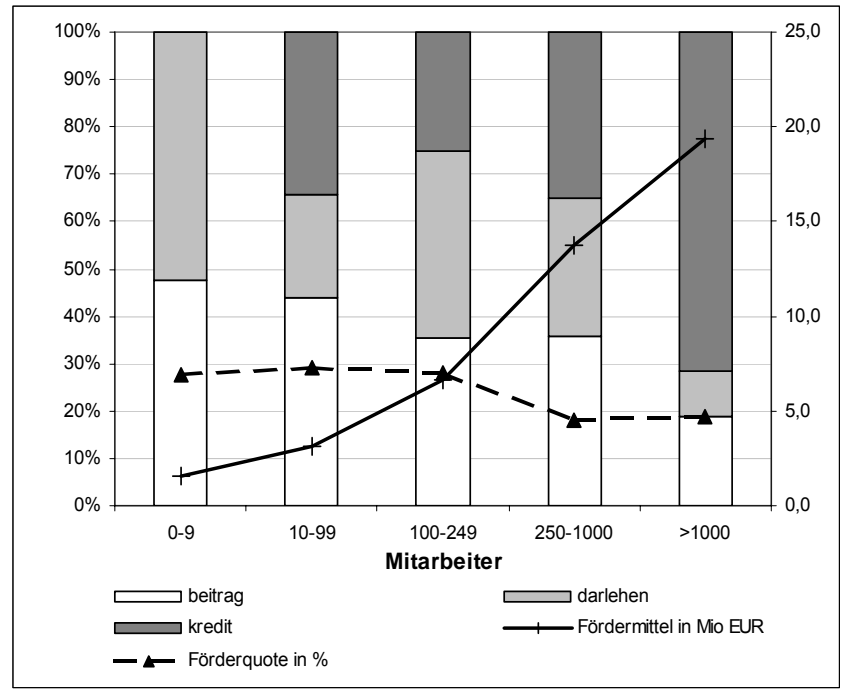

Quelle FFF, eigene Berechnungen

Abbildung 12 veranschaulicht die Verteilung der Fördermittel sowie den unterschiedlichen Fördermix in Mio Euro auf die Unternehmensklassen. Kleinere Unternehmen, die eher kleine Projekte einreichen erhalten mehr Zuschüsse, während größere Unternehmen mehr Darlehen des FFF und Sicherstellungen für Bankkredite erhalten. Obwohl die Förderquote als Barwert in \% der Projektkosten für größere Unternehmen niedriger ist als für kleine und mittlere Unternehmen, lukrieren größere Unternehmen dennoch das Gros der Fördermittel.

Insgesamt lukrierten die großen Unternehmen mit mehr als 250 MitarbeiterInnen 74,4\% der Fördermittel, während auf kleinere und mittlere Unternehmen 25,6\% der Fördermittel entfielen.

Im Interview mit dem FFF wies dieser darauf hin, dass die Beanspruchung der Initiative Mikrotechnik durch größere Unternehmen auch mit einer verstärkten Beratungstätigkeit des Fonds bei größeren Unternehmen zusammenhängt: Sie wurden explizit auf die Möglichkeiten der Initiative Mikrotechnik hingewiesen, um eine Veränderung im Forschungsportfolio dieser größeren Unternehmen zu bewirken.

\section{Fazit:}

Von den 63 Unternehmen (exklusive ARGEs) die Förderanträge in der Initiative Mikrotechnik gestellt haben, können 40 Unternehmen also rund 63\% der Gruppe der kleinen und mittleren Unternehmen zugerechnet werden. Zieht man die Anzahl der Bewilligungen in der Initiative Mikrotechnik als Maß für die Einbindung der kleinen und mittleren Unternehmen an der Initiative Mikrotechnik heran, so sinkt die Beteiligung der KMU an der Initiative auf 41\%. 
Wird zusätzlich nicht nur der Unternehmensanteil, sondern auch die Verteilung der Mittel als Maß für das Augenmerk oder die Hauptzielgruppe der Initiative berücksichtigt, so verlagert sich der Fokus der Initiative auf große Unternehmen: Große Unternehmen mit mehr als 250 MitarbeiterInnen lukrierten $74,4 \%$ der Fördermittel, während auf kleinere und mittlere Unternehmen 25,6\% der Fördermittel entfielen. Wir können leider keine Aussage darüber treffen, in welcher weise die Initiative eine Veränderung des Forschungsportfolios der Unternehmen bewirkt hat. 


\section{Relevanz - oder... ,macht der FFF überhaupt das Richtige?““}

\subsection{SCHWERPUNKTSETZUNG IM FFF}

Mit der Implementierung der Initiative Mikrotechnik hat der FFF einen (zumindest in der Außendarstellung seiner Tätigkeit) thematischen Schwerpunkt seiner Förderpolitik gesetzt. Dachs et al. 2003 haben eine Systematisierung von forschungs- und technologiepolitischen Schwerpunktsetzungen vorgenommen und unterscheiden drei wesentliche Typen: funktionale Schwerpunkte, wissenschaftlich-technologische Schwerpunkte und schließlich gesellschaftliche Schwerpunkte. Ein Schwerpunkt wie Mikrotechnologie gehört zu den wissenschaftlichtechnologischen Schwerpunkten. Dachs et. al unterscheidet hierin wiederum zwischen generischen Technologien mit Breitenwirkung (z. B. Informations- und Telekommunikationstechnologie, aber auch eben Nanotechnologie), fokussierten Nischen (Technologiefelder, in denen Österreich eine gewisse Prädisposition im Sinne von Stärken besitzt), und schließlich technologische Missionen (Überschallflugzeug Concorde, Transrapid). Schließlich kommen die AutorInnen zum Schluss: „Thematisch fokussierte Schwerpunktsetzung erscheint [...] in einer ganzen Reihe von wissenschaftlich-technologischen Forschungsfeldern auch in Österreich sinnvoll. Schwerpunkte in generischen wissenschaftlich-technologischen Bereichen sind auf Grund ihrer Ausstrahlungswirkung auf das für Österreich typische breite Spektrum an Anwenderindustrien wichtig.“

\section{Fazit:}

Mit der Fokussierung auf Mikrotechnologie hat der FFF gemäß den Ergebnissen der Studie „Zukunftspotentiale der österreichischen Forschung“ einen richtigen Zugang zur Setzung von Schwerpunkten in seiner Tätigkeit gewählt.

\subsection{MIKROTECHNIK - BASISTECHNOLOGIE}

Es war weder Aufgabe des Evaluierungsteams, neue Abgrenzungen zwischen Mikro- und Nanotechnologie zu finden, noch die Relevanz des Forschungsfeldes „Mikrotechnik“ an sich zu überprüfen. Es ist für eine Zwischenevaluierung allerdings eine legitime Fragestellung, inwieweit sich die Rahmenbedingungen, die zur Implementierung der Aktionslinie Mikrotechnik führten, verändert haben oder immer noch von Relevanz sind.

Der FFF hat mit der Beauftragung der Studie „Mikrotechnik in Österreich“ von C. Kukla im Jahre 2000 quasi eine feasibility study (wenn man so will eine ex ante Evaluierung der Initiative) vorgenommen. Darin wird Mikrotechnik als „Schlüsseltechnologie“ bezeichnet, als „zukunftsweisend“, deren Bedeutung in allen wichtigen Ländern wahrgenommen wird und auch zunimmt. Diese Bedeutung spiegelt sich auch in unterschiedlichen nationalen Schwerpunktsetzungen wieder, wie Box 3 weiter unten verdeutlicht. 
Box 3: Mikrotechnik in nationalen Schwerpunktsetzungen . Ein Exkurs

Aus einem Vergleich von IT-Schwerpunkten in ausgewählten europäischen Ländern ${ }^{6}$ ergibt sich, dass Mikrotechnologie auf dem Forschungsagenda aller untersuchten Ländern (D, NL, SWE, FIN, EU) zu finden ist. Oft ist der Schwerpunkt als Kombination 'Mikro- und Nanotechnologie' definiert, manchmal gibt es einen separaten Schwerpunkt 'Nanotechnologie'.

Deutschland hat im Rahmen des BMBF-Programms „IT-Forschung 2006“ vier Schwerpunkte gesetzt, Nanoelektronik ist einer davon und mit 395, 5 Mio Euro für den Zeitraum 2002-2006 dotiert. Das BMBF unterhält darüber hinaus das Programm „Mikrosystemtechnik“, das mit ca. 290 Mio Euro für den Zeitraum 2004-2009 dotiert ist. In der finnischen TEKES Technologiestrategie werden 6 Schwerpunkte identifiziert, davon ist ein Schwerpunkt Mikrosysteme. Nanotechnologie wird von TEKES als eines der langfristigen Forschungsbereiche definiert. In den Niederlanden ist "Nanotechnologie" einer der Prioritäten des dortigen Wirtschaftsministeriums. Zusätzlich hat das Wirtschaftsministerium „Mikrosystemtechnologie“ als eine Zukunftstechnologie definiert, auf die sie sich konzentrieren möchte. In Schweden hat VINNOVA insgesamt 18 Schwerpunktbereiche definiert, 3 davon sind im Bereich IT. Einer der Schwerpunkte ist "Mikro- und Nanoelektronik". Auch in Korea ist Nanotechnologie ein Schwerpunkt, es gibt sogar einen "Comprehensive Plan for the Development of Nanotechnology (2001)". Auch im "21st Century Frontier R\&D Program", das Projekte mit 10-Jahres Horizont unterstützt, ist Nanotechnologie ein Schwerpunkt. Mikrotechnologie wird in Korea nicht als Schwerpunkt angegeben.

Im "Information Society Technologies" Programm des 6. EU Rahmenprogramms ist "Mikrosysteme und Komponenten" eines der vier technologischen Schwerpunkte. Im 1. Call war „Mikro- und Nanosysteme“ als eines der strategischen Ziele (strategic priorities) ausgewählt.

Ob dieser Schluss auch vier Jahre später noch zutreffend ist, war Gegenstand einiger Interviews bzw. Rechercheschritte im Rahmen dieser Evaluierung. Wesentliche Aussagen zur Aktualität von Mikrotechnik waren unter anderem: „Mikrotechnik hat natürlich Zukunft. In Österreich besteht allerdings ein beträchtlicher Aufholbedarf, wobei es vor allem um die Umsetzung und Anwendung bestehenden Wissens geht.“ „Meiner Ansicht nach hat das Thema - wegen des in Österreich vorhandenen Wissens bei Forschern und bei industriellen Anwendern - weiterhin Bedeutung und sollte daher auch bei Forschungsförderungsaktionen berücksichtigt werden.“ Oder: „Mikrotechnik als Schwerpunkt ist besser als Nano oder Life Sciences.“

Durchsucht man die Literatur nach Hinweise, ob Mikrotechnik als Technologiefeld nun seinen „Zenit“ erreicht hat oder weiter wächst, werden durchwegs Wachstumspotentiale skizziert: „The MST market is forecast to grow 20\% annually“, 2005 wird der Weltmarkt für

\footnotetext{
${ }^{6}$ Weber, M. et al (2004), "IT-Heuristik", ARC sys und Joanneum Reserach. Studie (im Erscheinen) im Auftrag des bmvit. Schindler, J. (2004), "STI-Priority Setting: Country Study Korea", Joanneum Research. Included in: Gassler, H., Weber, M. et al (2004), "STI-Priority Setting: Cross-Country Survey", Joanneum Research and ARC sys.
} 
MST-Produkte 68 Milliarden \$ ausmachen”, „Mikro- und Nanotechnologie werden „die Schrittmacher des Fortschritts weit vor IT und Elektronik ${ }^{\star 6}$, , „Mikrotechnik ist längerfristig durch ein außerordentliches Wachstum mit großen wirtschaftlichen Potentialen geprägt. “99

\section{Fazit:}

Das Evaluierungsteam konnte keine Hinweise dafür entdecken, dass Mikrotechnologie ihren „Zenit“ überschritten oder gar als „,abklingender Trend“ zu gelten hätte. Interviews, Marktstudien und das Gewicht der Mikrotechnik in nationalen und international forschungs- und technologiepolitischen Programmen geben Hinweise darauf, dass ihr Platz auf der österreichischen technologiepolitischen Agenda durchaus berechtigt ist.

\subsection{ENTSPRICHT DIE KONKRETE ZIELFORMULIERUNG DER INITIATIVE DEN BEDÜRFNISSEN DER WIRTSCHAFT UND GESELLSCHAFT?}

Der technologische Fortschritt bildet die Basis des wirtschaftlichen Wachstums- und Wohlfahrtspotentials. Investitionen in Forschung und Technologieentwicklung zeigen enorme positive Effekte auf Wirtschafts-, Wohlfahrts- und Standortentwicklung. Ein Schluss, der in der Fachliteratur breit abgesichert ist. Warum hier öffentliche Mittel einsetzen? Das Vertrauen in ein freies Spiel der Marktkräfte, ein ,laissez-innover" privater Akteure, ist im Bereich der Forschungs- und Technologiepolitik unbegründet. Die von privatwirtschaftlichen Akteuren angebotene Menge an technischem Wissen liegt aufgrund bestimmter Mechanismen des Markt- und Systemversagens unter dem sozialen Optimum. Dies macht den Einsatz öffentlicher Mittel zur Förderung von Forschung und Technologieentwicklung notwendig. Staatliches Handeln ist gefordert, um die positiven Effekte, die Investitionen in Forschung und Technologieentwicklung auf Wirtschaft und Gesellschaft haben können, auch zu lukrieren.

Mission und Ziele der Initiative Mikrotechnik (vgl. hierzu Abschnitt 2.1 oben) entsprechen der oben skizzierten Rationalität des Mitteleinsatzes: Einerseits haben sie die Stoßrichtung, die Verwendung von Mikrotechnik zu dynamisieren und zu verbreitern, andererseits sprechen sie explizit Elemente des Markt- und Systemversagens an („Kooperation Wissenschaft Wirtschaft), aus denen heraus staatliches Handeln begründet wird.

Mikro- und Nanotechnologie wird unterstellt, ein Zukunftsmarkt zu sein (vgl. hierzu C. Kukla) und andere Märkte zu beeinflussen. Ein Programm wie die Initiative Mikrotechnik stellt einen Beitrag dar, Österreich auf diesen erwarteten Zukunftsmarkt zu positionieren.

Schließlich wurde der Befund, Mikrotechnologie sei ein Zukunftsmarkt, nicht nur für Österreich getroffen, sondern in vielen Ländern weltweit (vgl. Box 3). Somit kann man argumentieren, dass Programme wie die Initiative Mikrotechnik die Anschlussfähigkeit der nationalen Forschung an internationale Trends unterstützt. Nur wer Forschung in wichtigen Feldern wie Bio- und Nanotechnologie oder IKT auf einem hinreichend hohen Niveau betreibt, kann auch Erkenntnisse der Spitzenforschung aus dem Ausland nutzen. Derartige "secondmover" Strategien können durchaus erfolgreicher sein als Versuche, die

\footnotetext{
${ }^{7}$ Beide Aussagen aus: NEXUS Market Analysis 2002

${ }^{8}$ Schlüsseltechnologien 2010, Seite $5 \mathrm{ff}$.

${ }^{9}$ ecmc 2004, Seite 40
} 
Schrittmacherrolle zu übernehmen. Diese Anschlussfähigkeit ist auch die Voraussetzung, um von internationalen Forschungsprojekten wie den Rahmenprogrammen profitieren zu können. Für das nationale Forschungsportfolio eines kleinen Landes bedeutet dieser Ansatz, dass auch bei einem Bemühen um eine ausgewogene Balance zwischen Spezialisierung und Diversität des Forschungsportfolios eine Fokussierung auf einige wenige Bereiche mit hoher internationaler Anschlussfähigkeit anzustreben ist.

\section{Ein Blick über die Grenzen: welche Charakteristika zeigen Technologieförderprogramme?}

In den letzten Jahren haben sich in den meisten europäischen Ländern der Charakter von Technologieprogrammen verändert. Neben der Entwicklung neuer Technologien durch die Bündelung der (nationalen) F\&E-Ressourcen auf bestimmte Technologien übernimmt die Programmförderung heute eine Vielzahl weitere forschungs- und innovationspolitischer Zielsetzungen. Damit hat sich das Spektrum (neben den organisatorischen Notwendigkeiten eines Technologieprogramms wie Programmmanagement, Abstimmungsbedarf mit anderen Programmen, verbindliche und klare Zielsetzungen etc.) erweitert mit der entsprechenden Anpassung der einzelnen Förderinstrumente:

Förderung der Zusammenarbeit Wissenschaft und Wirtschaft: dies zählt heute zu einer der wichtigsten Instrumente der Förderung neuer Technologien. Hier lässt sich auch am deutlichsten ein Marktversagen als Legitimierung der Förderung zeigen.

Integration verschiedener Technologien, Multidisziplinarität ${ }^{10}$ : Technologieprogramme werden zunehmen integrativer, d.h. auf die Verbindung verschiedener Technologiefelder, Disziplinen und Sektoren ausgerichtet, z.B. in Bezug auf die Anwendung bestimmter Verfahren in der Materialentwicklung.

Bildung von regionalen Clustern: Technologieprogramme werden zunehmend für die Erreichung von regionalpolitischen Zielsetzungen genutzt, insbesondere für die Bildung von regionalen Technologieschwerpunkten im Rahmen von Clusteransätzen.

Förderung junger, technologieorientierter Unternehmen: neben den traditionellen Hauptadressaten von Technologieprogrammen (große, forschungsintensive Unternehmen) treten immer mehr auch kleine, technologieorientierte Unternehmen. Diese werden vor allem durch eine gezielte Erhöhung des KMU-Anteils in den Förderprogrammen erreicht.

Netzwerkbildungen: in Technologieprogrammen wird verstärkt der Gedanke der Netzwerkbildung zwischen den Akteuren im Innovationssystem betont, insbesondere als vertikale Netzwerke auf verschiedenen Ebenen der Wertschöpfungskette.

Förderung der Internationalisierung: vor allem in kleinen Ländern dient die Programmförderung auch der Stärkung der internationalen Vernetzung von Unternehmen und Forschungseinrichtungen in der Technologieentwicklung, insbesondere mit dem Ziel, Innovationsimpulse aus dem Ausland und Absatzpotentiale im Ausland zu nutzen.

Stärkung der Innovationskompetenz von KMU: als immer wichtiger wird die Stärkung der Innovationsfähigkeit von KMU gesehen. Dies soll zum Beispiel durch ,Lernen von

${ }^{10}$ Gerade der Mikro- und Nanotechnologie wird ein multidisziplinärer Charakter nachgesagt. 
großen Unternehmen' im Rahmen einer Zusammenarbeit zwischen KMU und größeren Unternehmen in Projektverbünden oder durch spezielle Programmmaßnahmen zur Erhöhung der organisatorischen und Prozesskompetenz in KMU erreicht werden.

\subsection{WURDEN DIE WIRTSCHAFTLICHEN UND GESELLSCHAFTLICHEN RAHMENBEDINGUNGEN BERÜCKSICHTIGT?}

Wir wollen in diesem Abschnitt untersuchen, in wie weit die Initiative Mikrotechnik, bzw. das Themenfeld „Mikro- und Nanotechnologie“ sich in verschiedenen Ansätzen einer österreichischen FTE-Strategie und anderen zentralen Dokumenten (Grünbuch, NaFIP, Regierungsprogramme, FTE - Berichte, etc.) wiederfindet. Wir wollen so Auskunft darüber geben, ob eine Schwerpunktsetzung im Bereich dieser Technologien den Linien von Regierung bzw. Rat widerspricht oder ob sie sich einbettet.

\section{Grünbuch}

Im Jahre 1999 steht Mikro und Nanotechnologie noch nicht auf der österreichischen (Forschungs-)politischen Agenda: Im Grünbuch zur österreichischen Forschungspolitik ${ }^{11}$ finden die Bereiche noch keinerlei Erwähnung. Allerdings werden „Kooperationen“ mit einem eigenen Kapitel vermehrt Aufmerksamkeit gewidmet.

\section{Regierungsprogramm 2000-2003 bzw. 2003 - 2006}

Auch im Regierungsprogramm 2000 - 2003 finden Mikro- und Nanotechnologie keine explizite Erwähnung. Hingegen wird der Fokus auf Bio- und Gentechnologie gelegt. Diese stellen „neben anderen Fachgebieten“ (wobei unklar bleibt, welche diese sind, und ob u.U. auch Mikro-Nanotech dazu zählt) wesentliche Forschungsschwerpunkte dar (vgl. Seite 67). Die Schwerpunktsetzung für die Förderungen der österr. Fonds wurde dem Rat für Forschung und Technologiepolitik übertragen. (siehe weiter unten). Auch sollte die nationale Forschungsförderung auf bestimmte Themenfelder konzentriert und mit entsprechenden EUProgrammen abgestimmt werden. Im aktuellen Regierungsprogramm werden keine spezifischen Forschungsfelder mehr genannt.

\section{Forschungs- und Technologieberichte der Bundesregierung}

In der Wahrnehmung der HerausgeberInnen und AutorInnen des Forschungs- und Technologieberichtes 2001 und 2002 scheint Mikro- und Nanotechnologie nicht auf; Hauptschwerpunkt der Betrachtungen liegt, was, bedenkt man den Zeitpunkt der Berichtslegung erklärlich ist, im Bereich Informations- und Kommunikationstechnologien. 2002 wird dies noch um „Biotechnologie“ ergänzt.

Im Bericht 2003 finden fünf Wissenschaftszweige Erwähnung, die - nach einer bibliometrischen Analyse - im internationalen Vergleich überdurchschnittliche ImpactFaktoren aufweisen. Zu diesen zählen Informatik, Molekularbiologie, Pharmakologie, Physik und Multidisziplinäre Zeitschriften. Hier muss man zu bedenken geben, dass Mikro- und Nanotechnologie Querschnittsmaterie darstellen und von solchen Analysen nur schwer zu

${ }^{11}$ bmwv 1999 
fassen sind. Außerdem wird im Technologiebericht 2003 auf die Ratsempfehlung, sich in Forschungs- und Technologiepolitik auf bestimmte Themenfelder $\mathrm{zu}$ fokussieren, auf Themenfelder zu denen auch Mikro- und Nanotechnologie zählen verwiesen. 2004 wird der Schwerpunktsetzung in Forschungs- und Technologiepolitik ein eigenes Kapitel gewidmet.

\section{Ratsempfehlungen, Nationaler Forschungs- und Innovationsplan NaFIP}

Der Rat für Forschung und Technologieentwicklung hat als wesentliches Element seiner Tätigkeit mit Schwerpunktsetzungen bzw. der Definition von Stärkefeldern operiert, nicht nur im NaFIP sondern auch in diversen anderen Dokumente:. „Moderne Forschungs- und Technologiepolitik muss Schwerpunkte setzen. Vorhandene Stärken sind zu stärken, Hoffnungspotentiale an die internationale Spitze heranzuführen." ${ }^{\text {12 }}$ Es wird eine Priorisierung in Anlehnung an die Rahmenprogramme auf fünf Forschungsfelder vorgenommen. $\mathrm{Zu}$ diesen Schwerpunkten zählen auch Nano- und Mikrotechnologie. Auffällig ist, dass in den verschieden Dokumenten nach Nennung der „Mikro- und Nanotechnologie“ als Schwerpunkt nur mehr von „Nanotechnologie“ die Rede ist, die als „vielversprechendste Zukunftstechnologie“ mit vergleichbaren positiven Effekten wie die IKT bezeichnet wird (vgl. S. 44f, NaFIP). Des weiteren wurden Strategieelemente für eine F\&E-Politik in Österreich entwickelt. Zu den Strategieelementen gehören unter anderem:

- Konzentration auf Aktivitäten mit großer Hebelwirkung von öffentlichen auf private Mittel

- Stärkung von Stärken- und Zukunftsfeldern

- Forcierung von Kooperationen

- Schaffen und Verstärken des Bewusstseins für FTI

- Stärkung der Humanressourcen und Informationstechnologie

- Förderung innovativer Start-ups

- Setzen von regionalen Schwerpunkten im Rahmen des entstehenden europäischen Forschungsraumes.

\section{Fazit:}

Die Initiative Mikrotechnik entspricht den Orientierungspunkten, die der Rat für Forschung und Technologieentwicklung in seinem Nationalen Forschungs- und Innovationsplan vorgegeben hat. Ein Schwerpunkt Mikrotechnik passt sich in die österreichische Forschungs- und Technologiepolitik ein; eine Initiative in diesem Bereich konterkariert die wirtschaftlichen und gesellschaftlichen Rahmenbedingungen nicht.

\subsection{MIKRO \& NANO FÖRDERUNG IM INTERNATIONALEN KONTEXT}

In diesem Abschnitt wird ein Spotlight auf das Förderanbot der EU im Bereich Mikro- und Nanotechnik geworfen um die Frage nach dem Bedarf nationaler Programme ansatzweise zu erörtern.

${ }^{12}$ Rat 2001 A, aber auch Rat 2001 B, Rat 2002 


\section{Mikro- \& Nanotechnologie im 6. Rahmenprogramm}

Im 6. Rahmenprogramm für Forschung und Entwicklung der Europäischen Union (20022006) stellen die Bereiche Nanotechnologien und Nanowissenschaften, wissensbasierte multifunktionelle Werkstoffe, neue Produktionsverfahren und -anlagen eine von 6 thematischen Prioritäten dar. Hierbei liegt der Fokus in der Nanotechnologie, da sie an der Schnittstelle von Quantentechnik, Werkstofftechnik und Molekularbiologie angesiedelt ist und - nach Auffassung der Kommission - ein „Schlüsselbereich der nächsten industriellen Revolution" sein dürfte, die beträchtliche Investitionen erfordert.

In Europa ist nach Ansicht der Europäischen Kommission zwar umfangreiches Know-how in bestimmten Bereichen wie der Nanofertigungstechnik und der Nanochemie vorhanden, die Anstrengungen in diesem Bereich sollen jedoch verstärkt und koordiniert werden. Im Bereich der Werkstoffe sollen intelligente Werkstoffe entwickelt werden, die bei ihrer Anwendung etwa in den Bereichen Verkehr, Energie, Elektronik und Biomedizin einen hohen Mehrwert erbringen sollen und für die es einen potenziellen Markt im zweistelligen EuroMilliardenbereich erwartet wird. Die Förderung erfolgt für Forschungseinrichtungen und Unternehmen in europäischen Forschungsnetzwerken.

In Österreich erfasst PROVISO die Beteiligung an den Programmlinien des 6. Rahmenprogramms. In den bisherigen Ausschreibungen zur Nanotechnologie wurden bisher europaweit 1018 Projekte evaluiert (Stand 27.07.04) und 131 dieser Projekte für eine Förderung vorgesehen. An knapp einem Drittel dieser Projekte (289) haben sich österreichische Partnerorganisationen beteiligt. Die Priorität Nanotechnologie zeichnet sich durch eine hohe Überzeichnungsrate aus und verspricht dementsprechend eine geringe Erfolgsquote. Mit einer Erfolgsquote von 12,8\% liegt Österreich im europäischen Schnitt.

Was den Anteil an Beteiligungen auf Basis der Organisationen betrifft entfallen auf Österreich 2,3\% der evaluierten und 2,1\% der erfolgreichen Beteiligungen. Betrachtet man die Beteiligung nach Organisationsform, so weicht die Erfolgsquote für österreichische KMU mit 4\% von der Gesamtquote für europäische KMUs die bei $12 \%$ liegt ab, während die Erfolgsquote für große Unternehmen im europäischen Schnitt der Großunternehmen liegt.

\section{Mikrotechnologie in EUREKA}

EUREKA ist kein zentral organisiertes Förderprogramm, sondern eine Netzwerkinitiative die Industrie und Wissenschaft einen Rahmen für grenzüberschreitende Kooperationsprojekte gibt. Die Finanzierung erfolgt auf nationaler Ebene - durch nationale Fördermittel, öffentliche Kredite oder durch eigene Mittel. In ihrer Finanzierungsstrategie werden die Projektteilnehmer von den nationalen EUREKA Büros unterstützt. Das österreichische EUREKA Büro liegt beim BIT.

Innerhalb von EUREKA forciert EURIMUS (http://www.eurimus.com) die industrielle Nutzung von Mikrosystemen bzw. Kommerzialisierung der Mikrosystemtechnologie als eines von sechs Clusterprojekten, welches Kooperationen europäischer KMU - Unternehmen unterstützt. Die Projektvorhaben sind in elf vorgegebenen, jedoch weitgefassten Marktsegmenten (z.B. Umwelt, Luft- und Raumfahrt, Medizin, Multimedia, Transport etc.) anzusiedeln. 
An EURIMUS Projekten müssen sich mindestens zwei Industrie-Unternehmen aus unterschiedlichen EUREKA-Mitgliedsländern und Forschungsinstitute bzw. Hochschulen zu einem Konsortium zusammenschließen. Ein Business Plan mit Produktentwicklungen und dem geplanten ROI in den einzelnen Ländern ist zu erstellen, um die Zukunftsperspektiven des Unternehmens und das Potential des Vorhabens zu erörtern. EURIMUS Projekte haben eine Laufzeit von 2-3 Jahren und Projektvolumen in der Größenordnung von 1-20 Mio $€$ (Durchschnitt 6 Mio $€$ ).

\section{Fazit:}

Während der Schwerpunktbereich Nanotechnologie im 6. Rahmenprogramm sehr große, grundlagenorientierte Forschungsprojekte fördert und durch eine hohe Ablehnungsquote gekennzeichnet ist und keinen Anknüpfungspunkt für den FFF bietet, stellt die EURIMUS Initiative einen möglichen Anknüpfungspunkt für die FFF-Initiative Mikrotechnik dar: Sie ist von der Finanzierungsseite gesehen auf nationale Förderstellen angewiesen ist, legt einen Schwerpunkt auf grenzüberschreitende WissenschaftWirtschaft Kooperation mit Netzwerkcharakter, dient als Facilitator für die Partnersuche von Unternehmen, und kann für Fördergeber und Empfänger als Label für anspruchsvolle Forschung im internationalen Verbund genutzt werden. Durch die Zusammenführung von FFF \& BIT in der FFG ergeben sich neue, zu nutzende Kooperationspotentiale für den FFF.

\subsection{DIE INITIATIVE MIKROTECHNIK IM VERGLEICH ZUR MIKROSYSTEMTECHNIK 2000+ UND ZU TOP NANO 21}

In diesem Abschnitt folgt eine kurze systematische Darstellung des deutschen Programms Mikrosystemtechnik 2000+ und des Schweizer Programms TOP NANO 21 mit dem Ziel, einen Einblick in die Programmgestaltung und Schwerpunktsetzung von mit der Initiative Mikrotechnik vergleichbaren Programmen zu erhalten.

\section{Mikrosystemtechnik 2000+}

Das BMBF (Bundesministerium für Bildung und Forschung) initiierte das Programm „Mikrosystemtechnik 2000+“, um die bereits bestehende Förderreihe im Bereich der Mikrotechnik fortzusetzen. Der Projektträger VDI/VDE-Innovation + Technik GmbH legte den Schwerpunkt des Förderkonzeptes auf die Zusammenarbeit zwischen Industrie und Wissenschaft. Es werden besonders die Probleme der KMUs und ihre darausfolgende Unterstützung dabei berücksichtigt.

Das BMBF Programm „Mikrosystemtechnik 2000+“ startete im Jahr 2000 und führte damit die bereits seit 1990 bestehende systematische Förderung der Mikrosystemtechnik (MST) weiter fort. Dabei erfolgte eine deutliche Schwerpunktverlagerung von der Forschung zur Entwicklung und Anwendung.

Verbundvorhaben als Kooperationen zwischen Partnern aus der Wissenschaft und der Wirtschaft haben sich folglich zu Gunsten der Beteiligung industrieller Partner verschoben. In den Programmphasen II (1994-1999) und III (ab 2000) kommen mehr als zwei Drittel der Partner aus Unternehmen und hier insb. aus KMU mit einer Beteiligungsquote von $61 \%$. 
Die Evaluation des Programms Mikrosystemtechnik 2000+ zeigte, dass das Ziel inhaltlich für neue Entwicklungen offen zu bleiben und branchen- bzw. disziplinenübergreifende FuEKooperationen zu ermöglichen sehr gut bewältigt wurde.

Bei über 40 \% der Förderfälle wurden neue F\&E-Verbünde initiiert und die Zusammenarbeit intensiviert. Seit dem Jahr 2000 ist ein zunehmend breiterer Branchenmix bei den Antragstellern feststellbar ist (vgl. Pressemitteilung des BMBF 135/2001, der auf einen signifikanten Anstieg von Unternehmen der Pharma- und chemischen Industrie verweist).

Durch das MST Förderprogramm ist es insbesondere gelungen, so die Evaluierungsstudie, KMU einen zukunftsweisenden Technologiebereich zu öffnen. MST-Komponenten und Produkte werden zum Großteil in KMU geschaffen bzw. angewendet, was sich auch in der hohen Beteiligungsrate dieses Unternehmenstyps wiederspiegelt (fast die Hälfte der Verbünde setzen sich aus Partnern von KMU und FuE-Einrichtungen zusammen).

Trotz der positiven Wirkung der bisherigen Förderung im Bereich MST-Know-How und Wettbewerbsfähigkeit bestehen noch immer Interventionsbedarf. Deshalb wurde das Rahmenprogramm Mikrosysteme (2004-2009) implementiert, und hier ganz bewusst eine Schwerpunktsetzung vorgenommen. Ein bevorzugter Fokus liegt weiterhin auf KMU.

Eine flankierende Maßnahme ist die 2002 initiierte Maßnahme „Aus- und Weiterbildung in der MST", welche den Nachschub qualifizierten Personals und die interdisziplinäre Zusammenarbeit durch eine dreijährige Förderung von zunächst sechs Ausbildungsnetzwerken sicher stellen will. Ferner werden Fördermittel für eine Verzahnung dieser Ausbildungsnetzwerke bereit gestellt (vgl. Pressemitteilung des BMBF 75/2002).

\section{TOP NANO 21}

Das schweizerische Projekt „TOP NANO 21“ wurde vom ETH-Rat (Rat der eidgenössischen technischen Hochschulen) geschaffen, um eine Stärkung der schweizerischen Wirtschaft durch die Entwicklung und die Anwendung der Nanotechnologie zu erzielen. Der wichtigste Grundsatz der KTI, die für die operative Umsetzung zuständig ist, verbindet die drei Dimensionen Grundlagenforschung (Erkenntnisse), Technologieentwicklung und Anwendungen miteinander.

Die Initiative TOP NANO 21 legt als entscheidendes Förderkriterium die Kooperation zwischen einem Non-Profit-Forschungszentrum und mindestens einem Wirtschaftspartner fest. Wird die Projektleitung vom Forschungszentrum übernommen, kann dieses mit einer Förderung bis zu $100 \%$ rechnen. Liegt die Leitung hingegen beim Wirtschaftspartner ${ }^{13}$, wird bis zu 50\% gefördert. Weiters setzt das Programm eine Verknüpfung der drei Dimensionen Grundlagen, Technologien und Anwendungen innerhalb der einzelnen Projekte voraus. Die Erkenntnisse der Nanotechnik sollen über Netzwerke verstärkt zu den Fachhochschulen getragen und folglich in die Lehrinhalte integriert werden.

\section{Fazit:}

Tabelle 10 stellt die Initiative Mikrotechnik, das Rahmenprogramm Mikrosysteme (Mikrosystemtechnik 2000+) und die TOP NANO 21 Initiative schematisch gegenüber und macht Unterschiede und Gemeinsamkeiten im Programmdesign erkennbar. Während

\footnotetext{
${ }^{13}$ Aus ordnungspolitischen Gründen kommt es in der Schweiz prinzipiell zu keinen Geldflüssen zwischen Forschungsund Technologieförderung und Unternehmen.
} 
Mission und Zielsetzungen durchaus ähnlich sind, setzen TOP NANO 21 und die Mikrosystemtechnik 2000+ im Gegensatz zur FFF-Initiative klar auf Forschungskooperationen zwischen Unternehmen und Forschungseinrichtungen. Als Reaktion auf die vorherrschende Wirtschaftsstruktur in Deutschland nahm das Programm MST 2000+ bei seiner Förderung besonders auf die KMUs Rücksicht - durch branchenübergreifende Kooperationen und Unterstützung beim Aufbau der Infrastruktur soll den Problemen der KMUs entsprochen werden - und im Rahmenprogramm Mikrosystemtechnik 2004-2009 wird auch eine thematische Schwerpunktsetzung vorgenommen . 
Tabelle 10: Die Initiative Mikrotechnik im internationalen Vergleich

\begin{tabular}{|c|c|c|c|c|}
\hline & Initiative Mikrotechnik (A) & TOP NANO 21 (CH) & Rahmenprogramm Mikrosysteme (D) \\
\hline & & http://www.fff.co.at & http://www.bbt.admin.ch/ & http://www.mstonline.de/ \\
\hline \multicolumn{2}{|c|}{$\begin{array}{l}\text { Programm- } \\
\text { management }\end{array}$} & Forschungsförderungsfonds (FFF) & Kommission $\mathrm{f}$. Technologie $\mathrm{u}$. Innovation & VDI/VDE Innovation + Technik GmbH \\
\hline \multicolumn{2}{|c|}{ Mission } & $\begin{array}{l}\text { Verankerung und Weiterentwicklung von } \\
\text { Mikrotechnik in Österreich auf breiter } \\
\text { Basis (insbesondere KMU) }\end{array}$ & $\begin{array}{l}\text { „Damit die Schweizer Wirtschaft zügig neue, auf } \\
\text { dem Nanometer basierende Technologien nutzt } \\
\text { und in innovative Produkte und Dienstleistungen } \\
\text { entwickelt“ }\end{array}$ & $\begin{array}{l}\text { „Beschleunigung von Innovationsprozessen durch } \\
\text { Minderung von Innovationsbarrieren auf } \\
\text { einzelbetrieblicher und gesamtwirtschaftlicher } \\
\text { Ebene“ }\end{array}$ \\
\hline \multicolumn{2}{|c|}{ Laufzeit } & $2000-2003$ & $\begin{array}{l}2000 \text { - 2003, wird im Regelprogramm der KTI } \\
\text { weitergeführt }\end{array}$ & $2004-2009$ \\
\hline \multicolumn{2}{|c|}{ Zielgruppe } & $\begin{array}{l}\text { Unternehmen in Kooperation mit } \\
\text { Forschungseinrichtungen möglich }\end{array}$ & $\begin{array}{l}\text { Unternehmen, Forschungseinrichtungen } \\
\text { (Förderempfänger) }\end{array}$ & $\begin{array}{l}\text { Unternehmen (besonders KMUs), } \\
\text { Forschungseinrichtungen }\end{array}$ \\
\hline \multicolumn{2}{|c|}{ Instrumente } & $\begin{array}{ll}\text { - } & \text { Projektförderung } \\
\text { - } & \text { Andere Aktionslinien des FFF }\end{array}$ & $\begin{array}{ll}\text { - } & \text { Projektförderung } \\
\text { - } & \text { Andere Aktionslinien der KTI/CTI (start } \\
\text { ups) } & \text { Aus- und Weiterbildung }\end{array}$ & $\begin{array}{ll}\text { - } & \text { Projektförderung } \\
\text { - } & \text { Aus- und Weiterbildung } \\
\text { - } & \text { Innovationsunterstützende Maßnahmen (road } \\
& \text { maps, Fachgespräche ...) }\end{array}$ \\
\hline
\end{tabular}




\subsection{POSITIONIERUNG DER INITIATIVE MIKROTECHNIK ZUR ,NANO AUSTRIA’ INITIATIVE}

Wir haben in der Einleitung darauf hingewiesen, dass der Übergang zwischen Mikrotechnik und Nanotechnologie ein fließender ist und dass die Definitionsarbeit rund um diese Begriffe noch nicht abgeschlossen ist. Wir versuchen daher, einen Vergleich der zwei Förderinstrumente Initiative Mikrotechnik und Nano Austria-Initiative nur auf Grundlage der Programmunterlagen und aus forschungs- und technologiepolitischen Blickwinkel zu ziehen.

Das mehrjährige Förderprogramm „NANO Initiative Österreich“ wird vom BMVIT in Zusammenarbeit mit mehreren Ministerien, Bundesländern und Förderstellen durchgeführt. Die ASA Austrian Space Agency leitet das Programm-Management.

Die Schaffung von Rahmenbedingungen für eine verstärkte Kooperation zwischen Wissenschaft und Unternehmen definiert sich als Hauptziel der NANO Austria Initiative. Dadurch soll einerseits die Wettbewerbsfähigkeit gesteigert werden und andrerseits eine Positionierung Österreichs in europäischen und internationalen Kooperationen erfolgen.

Die NANO Austria Initiative ist ein Programmbündel und umfasst vier Linien, die festgelegte Themenbereiche beinhalten. Projektanträge können nur zu bestimmten Stichtagen (calls) eingereicht werden, die einer externen internationalen Evaluierung unterzogen werden. Die Evaluierung erfolgt anhand von sechs Kriterien, die wiederum in mehrere Fragestellungen unterteilt sind. Um zu einem Förderungsübereinkommen zu gelangen, müssen mindestens die festgelegten Schwellenwerte erreicht werden.

- Forschung und Technologieentwicklung in Verbundprojekten. In mehrjährigen Projekten arbeiten Forschungseinrichtungen und Firmen zusammen, um aufbauend auf Erkenntnissen der Grundlagenforschung Verfahren und Anwendungen zu entwickeln.

- Netzwerke und Vertrauensbildung Nano Forum Austria: Eine Informations- und Kommunikationsplattform für alle Nano- Akteure. Darüber hinaus werden weitere Vernetzungsmaßnahmen unterstützt.

- Maßnahmen zur Aus- und Weiterbildung für Studierende, ForscherInnen sowie MitarbeiterInnen von Unternehmen (noch nicht implementiert) ${ }^{14}$

- Begleitmaßnahmen: Als Beispiele für Begleitmaßnahmen werden „Fachstudien, Markt- und Machbarkeitsstudien, strategische Konzepte, Technology Foresight und Analysen" genannt. ${ }^{15}$

In Tabelle 11 wird nun versucht, einen Vergleich zwischen der Initiative Mikrotechnik und der Nano Initiative Österreich hinsichtlich Mission, Ziele, Zielgruppen und Instrumente zu ziehen.

\footnotetext{
${ }^{14}$ Aus und Weiterbildung in thematische Programme zu verpacken ist en vogue (siehe FIT IT). Daneben gibt es in Österreich über 40 Stipendienprogramme, Doktoratsprogramme, Frauenförderungsprogramme etc. Der Rat für Forschung und Technologieentwicklung erarbeitet zur Zeit gerade eine Katalogisierung dieses Bereiches, dem eine Ordnung und Neuaufstellung gut täte.

${ }^{15}$ Hier sollte zumindest angemerkt werden, dass diese Linie in vielen Bereichen eine Duplizierung bestehender Frderprogramme ist: Feasability Studies des FFF (Machbarkeitsstudien), Tecnet der AWS (Marktstudien).
} 
Gleich vorweg: die Initiative Mikrotechnik ist in dem Bereich „Mikro- und Nanotechnologie“ in Österreich ,first mover', die Nano-Initiative , second mover'. Wenn diese Evaluierung zum Schluss käme, es gibt wesentliche Überschneidungen zwischen den beiden Programmen, kann dies nicht als Kritik am Initiativen-Design des FFF interpretiert werden.

\section{Die Initiative Mikrotechnik ist keine Duplizierung der Nano Austria Initiative.}

Es gibt wesentliche Unterschiede in den Zielgruppen und den Instrumenten: während der FFF (vorrangig) Unternehmen anspricht ${ }^{16}$ und dies in Projekten mit einjähriger Laufzeit tut, spricht Nano Unternehmen und Forschungseinrichtungen an und tut dies auf mehrjähriger Basis in den Verbundprojekten. FFF Projekte sind mit diesen Verbundprojekten nicht vergleichbar (Menge der PartnerInnen, inhaltliche Ausrichtung.). Darüber hinaus ist anzunehmen, dass das Projektvolumen der Verbundprojekte ungleich höher ist als in der Initiative Mikrotechnik.

\section{Die Nano Austria Initiatve dupliziert (in engen Teilbereichen) die Tätigkeiten des FFF}

Sehr wohl dupliziert die Nano Austria Initiative in engen Bereichen die Aktionslinien des FFF: Machbarkeitsstudien sind sowohl über die Programmlinie 4 der Nano Initiative förderbar, als auch über die Aktionslinie Feasibility Studies. Im Bereich der Ausbildung, die von der Nano Initiative noch nicht designt wurde, gibt es das Potential der Überschneidung mit der Nachwuchsförderung des FFF. Der guten Ordnung halber weisen wir darauf hin, dass hier mit anderen Einrichtungen (ÖAW, FWF) ein höheres Überschneidungspotential besteht.

\section{Die Definition von „Nano“ schließt die Behandlung mikrotechnischer Fragestellungen im Rahmen der Nano Initiative Österreich aus.}

„Der Begriff NANO umfasst sowohl die nanoskalierten Wissenschaften als auch die daraus resultierenden Nanotechnologien. NANO beschäftigt sich mit Systemen, deren neue Funktionen und Eigenschaften ursächlich von den nanoskaligen Effekten ihrer Komponenten abhängig sind. Die charakteristischen Größenordnungen dieser Komponenten liegen zwischen einigen $0,1 \mathrm{~nm}$ und einigen $100 \mathrm{~nm}$, wobei eine Abgrenzung meistens unscharf und themenbezogen bleibt.“17

\section{Die Nano Initiative schließt die Förderung von Projekten aus dem Mikrotechnikbereich explizit aus:}

„Eine Doppelförderung von FTE-Projekten ist grundsätzlich nicht möglich. Spricht ein Projekt ein Thema an, das von einem anderen Förderprogramm abgedeckt wird, sollte es dort für eine Förderung eingereicht werden. Das Programm-Management unterstützt alle Antragsteller bei der frühzeitigen Klärung dieser thematischen Zuordnung. Beispielsweise können Projekte, die wesentlich dem Mikro-Bereich zugeordnet werden, direkt beim FFF im Rahmen der Mikrotechnik-Initiative eingereicht und begutachtet werden. Auf keinen Fall werden diese

\footnotetext{
${ }^{16}$ „Forschung der Wirtschaft“, „Durch die Förderung von Forschung und Entwicklung stärken wir die Konkurrenzfähigkeit der im Land angesiedelten Betriebe“. Aus dem Leitbild des FFF und der Homepage.

${ }^{17}$ Leitfaden für AntragstellerInnen Austrian NANO Initiative, 1. Ausschreibung 2004 für FTE-Verbundprojekte, Seite 7
} 
Projekte zu einem signifikanten Anteil aus dem Budget der Österreichischen NANO Initiative gefördert. “'18

${ }^{18}$ Als solche Programme, die potentiell Gegenstand einer Doppelförderung sind, spricht die Nano Initiative neben Mikrotechnik GEN AU und FIT IT an. (BMBWK: http://www.gen-au.at, BMVIT: http://www.fit-it.at ). Ebenda, Seite 10 
Tabelle 11: Gegenüberstellung ,Nano Austria - Initiative’ ,Initiative Mikrotechnik'

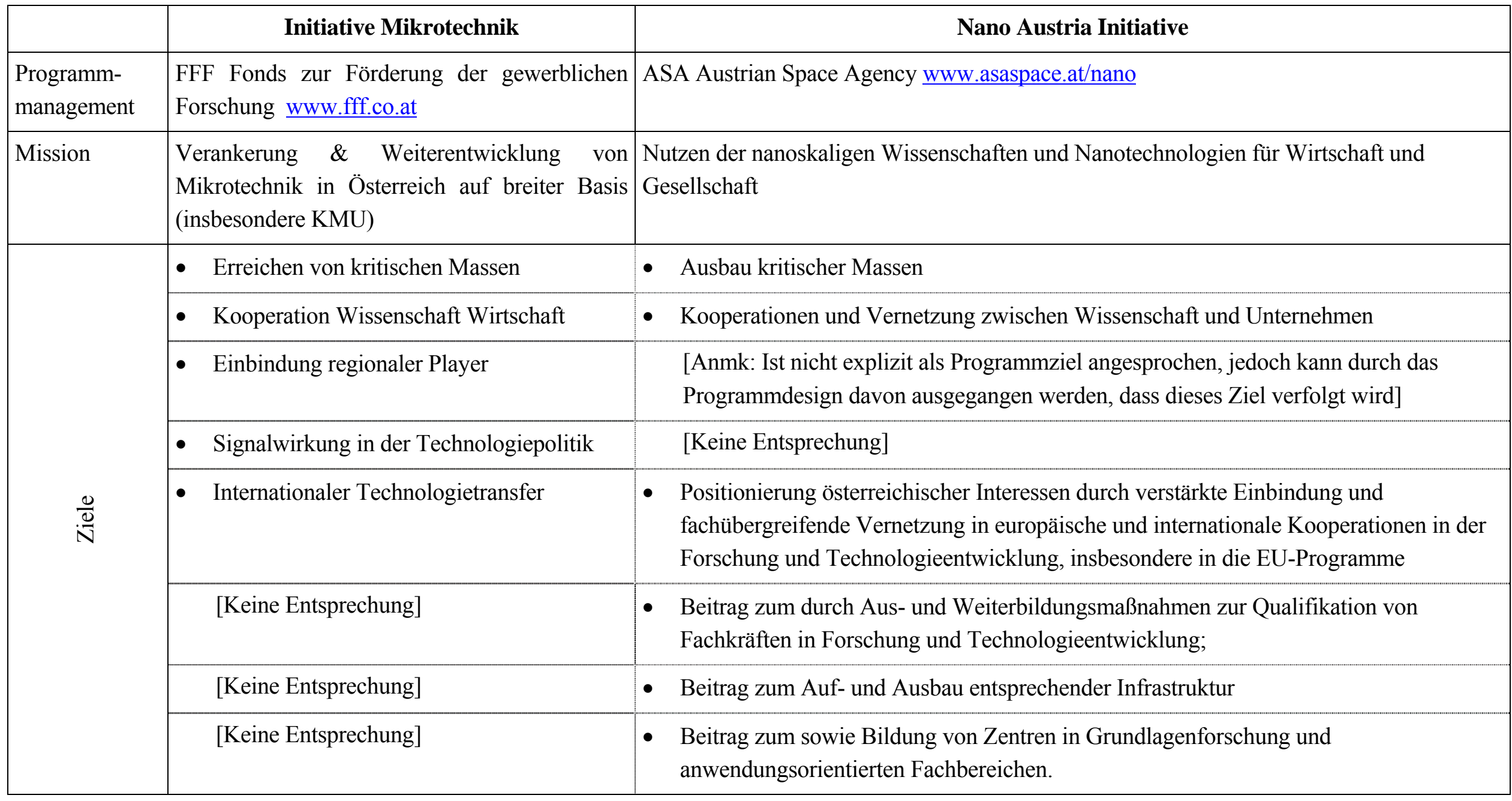




\begin{tabular}{|c|c|c|}
\hline & Initiative Mikrotechnik & Nano Austria Initiative \\
\hline Zielgruppen & Unternehmen & „sämtliche Nanoakteure“: Universitäten, außeruniversitäre Einrichtungen, Unternehmen \\
\hline \multirow{4}{*}{ 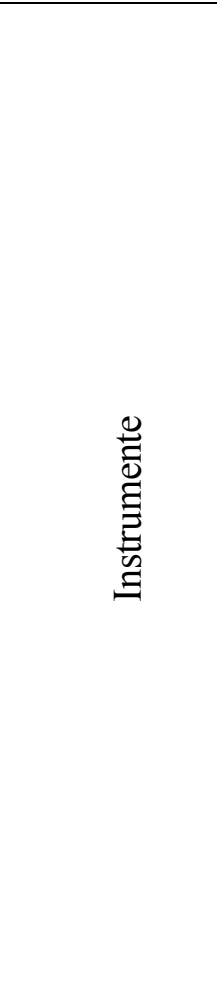 } & - Bottom-up Projektförderung & $\begin{array}{l}\text { - Forschung und Technologieentwicklung in Verbundprojekten } \\
\text { In mehrjährigen Projekten arbeiten Forschungseinrichtungen und Firmen um } \\
\text { aufbauend auf Erkenntnissen der Grundlagenforschung Verfahren und Anwendungen } \\
\text { zu entwickeln. }\end{array}$ \\
\hline & $\begin{array}{l}\text { - } \quad \text { ERA Net } \\
\text { - } \quad \text { Lenkungsausschuss }\end{array}$ & $\begin{array}{l}\text { - Netzwerke und Vertrauensbildung } \\
\text { Nano Forum Austria: Eine Informations- und Kommunikationsplattform für alle Nano- } \\
\text { Akteure. Darüber hinaus werden weitere Vernetzungsmaßnahmen unterstützt. }\end{array}$ \\
\hline & - FFF - Nachwuchsförderung & $\begin{array}{l}\text { - Maßnahmen zur Aus- und Weiterbildung } \\
\text { Aus- und Weiterbildungsmaßnahmen für Studierende, ForscherInnen sowie } \\
\text { MitarbeiterInnen von Unternehmen (noch nicht implementiert) }\end{array}$ \\
\hline & - Feasibility Studies & $\begin{array}{l}\text { - Begleitmaßnahmen } \\
\text { Als Beispiele für Begleitmaßnahmen werden „Fachstudien, Markt- und } \\
\text { Machbarkeitsstudien, strategische Konzepte, Technology Foresight und Analysen“ } \\
\text { genannt }\end{array}$ \\
\hline
\end{tabular}




\section{Initiative Mikrotechnik und NANO Austria - Initiative: Ergänzung?}

Wir glauben, dass auf Instrumentenebene die Initiatve Mikrotechnik eine Ergänzung zur Nano Austria - Initiatve darstellt: Auswahlprozess, Zahl der PartnerInnen und inhaltlicher Zuschnitt sind wesentliche Unterscheidungsmerkmale. Sieht man das Gesamtportfolio jener Fördermaßnahmen, die im Bereich der Mikro- und Nanotechnologie angesiedelt sind, so kann man also keine Überschneidungen erkennen und sehen ein wechselseitiges Verstärken in der Erreichung gemeinsamer Ziele wie der Schaffung kritischer Massen.

\section{Sind Synergieeffekte einer Zusammenführung dieser Initiativen festzustellen?}

Wir glauben, dass mögliche Synergieeffekte vor allem im Bereich der Awareness Maßnahmen und Vernetzungsaktivitäten zu finden sind: hier passiert sehr viel im Bereich der Nano Austria - Initiative (und darüber hinaus), Tabelle 12 fasst einige dieser Initiativen zusammen. Angesichts des bestehenden Anbots an Netzwerkinitiativen ist es wohl besser eher an den bestehenden Netzen anzudocken als neue Netze zu entwickeln.

Auch im Ansprechen von „Förderrookies“ ist es wohl einfacher, gemeinsam anstatt getrennt vorzugehen. Zwar duplizieren sich die beiden Initiativen nicht, doch für potentielle Neukunden wird es eher verwirrend sein, sich mit zwei „Marken“ auseinandersetzen zu müssen.

Eine „Marke“ „FFG -Nano \& Mikro“ wäre wohl in der Kommunikation nach außen sinnvoller, effizienter und billiger als eine Marke „FFG -Bereich 1 - (vormals FFF) Mikro (und Nano)“ bzw. „FFG - Bereich 3(vormals ASA) - Nano Österreich“

Mit der Leitung des MNT ERA-NETS leistet der FFF auch einen wesentlichen Beitrag zu der Positionierung österreichischer Interessen in der Gestaltung der Förderung von Forschung und Technologieentwicklung im Technologiebereich, ein Ziel, dem sich die Nano Initiatve verschrieben hat. 
Tabelle 12: Mikro- und Nano-Netze in Österreich

\begin{tabular}{|c|c|c|c|c|c|}
\hline & NANONET-Styria & $\begin{array}{c}\text { Micro@Nano-Fabrications- } \\
\text { Austria }\end{array}$ & w-INN & network- $\mu$ & $\begin{array}{c}\text { Nano Initiative } \\
\text { (Programmlinie Netzwerke und } \\
\text { Vertrauensbildung) }\end{array}$ \\
\hline & www.nanonet.at & http://www.unileoben.ac.at/ & $\underline{\text { www.hitt.at }}$ & http://www.polyconcent.at/protec.htm & www.asaspace.at \\
\hline Mission & $\begin{array}{l}\text { Koordination und } \\
\text { Bündelung der } \\
\text { Kompetenzen und } \\
\text { Konzepte der Nano- } \\
\text { wissenschaften und Nano- } \\
\text { technologien in der } \\
\text { Steiermark }\end{array}$ & $\begin{array}{l}\text { Entwicklung der } \\
\text { Marktaussichten in der } \\
\text { Nanotechnologie durch } \\
\text { verstärkte Organisation der } \\
\text { Netzwerk-Aktivitäten }\end{array}$ & $\begin{array}{l}\text { Internationale Schlüssel- } \\
\text { position in der Nano- } \\
\text { technologie durch den } \\
\text { Aufbau eines Netzwerkes }\end{array}$ & $\begin{array}{l}\text { Bedarfsorientierter Kompetenzaufbau } \\
\text { im Netzwerk von } 6 \text { Kleinunternehmen } \\
\text { unter starker Nutzung internationaler } \\
\text { Know-how aus Wirtschaft und } \\
\text { Wissenschaft }\end{array}$ & $\begin{array}{l}\text { „Bildung von neuen Netzwerken in } \\
\text { Ergänzung zu existierenden NANO } \\
\text { Netzwerken in Österreich“ } \\
\text { Generierung innovativer Ideen für } \\
\text { Forschungsprojekte }\end{array}$ \\
\hline $\begin{array}{l}\text { Thematische } \\
\text { Schwerpunkte }\end{array}$ & $\begin{array}{l}\text { - } \text { Nano-Coating } \\
\text { - } \text { Nano-Analytik } \\
\text { - } \text { Nano-Pulver } \\
\text { - } \text { Organische Opto- } \\
\text { Elektronik } \\
\text { - } \text { Bionanonet }\end{array}$ & $\begin{array}{l}\text { Ionenstrahl-Technik: } \\
\text { - „Bio-inspired“ Materialien } \\
\text { - funktionale Nano- } \\
\text { Materialien } \\
\text { - Nano-Elektronik sowie } \\
\text { Sensor- und } \\
\text { Aktuatorsysteme }\end{array}$ & $\begin{array}{l}\text { - Nanobiotechnologie } \\
\text { - Bio-Nanostrukturen } \\
\text { - Anorganische } \\
\text { - Nanostrukturen } \\
\text { - Bionanosensorik }\end{array}$ & $\begin{array}{l}\text { Technologieentwicklungen für } \\
\text { Spritzgussteile im Mikro- und } \\
\text { Nanobereich für High-tech } \\
\text { Anwendungen }\end{array}$ & $\begin{array}{l}\text { - Biologie } \\
\text { - Biotechnologie } \\
\text { - Chemie } \\
\text { - Elektronik } \\
\text { - Energietechnik } \\
\text { - Engineering } \\
\text { - Optik } \\
\text { - Physik etc. }\end{array}$ \\
\hline Beteiligte & $\begin{array}{l}\text { Steirische Forschungsein- } \\
\text { richtungen und } \\
\text { Industriebetriebe }\end{array}$ & $\begin{array}{l}\text { Wissenschafter und } \\
\text { Unternehmen aus Wien, NÖ, } \\
\text { Steiermark und OÖ }\end{array}$ & $\begin{array}{l}\text { Tiroler } \\
\text { Universitätsinstitute und } \\
\text { Unternehmen }\end{array}$ & Kleinunternehmen & $\begin{array}{l}\text { Unternehmen, Forschungsein- } \\
\text { richtungen, Fachhochschulen, } \\
\text { Höhere Technische Lehranstalten } \\
\text { sowie andere nano-relevante } \\
\text { Institutionen, etwa Vereine und } \\
\text { Gesellschaften }\end{array}$ \\
\hline
\end{tabular}




\section{Interne Kohärenz - oder... ,hat der FFF das richtige Design gewählt?““}

\subsection{ZUR ROLLE DES PROGRAMMKOMITEES}

Zur Begleitung der Initiative Mikrotechnik hat der FFF ein Programmkomitee eingereicht, das die Aktionslinie begleitete, den FFF in der Ausrichtung der Initiative beriet und schließlich auch operationalisierte Zielsetzungen entworfen hat. Das Programmkomitee traf sich in den Jahren 2000 und 2001 dreimal und hat dann seine Tätigkeit eingestellt. ${ }^{19}$

Der Analyse der Tagesordnungen ist zu entnehmen, dass das Programmkomitee keine unwesentliche Rolle im Design des Programme hatte; so wurden unter anderem besprochen

- Wie breit der Fokus des Programms gelegt werden sollte

- Ziele und Zielgruppen

- Gestaltung der flankierenden Maßnahmen

- Namensgebung (also ob auch ,Nanotechnologie' im Titel der Initiative aufscheinen sollte')

Allerdings ist die Verbindlichkeit des Besprochenen sehr unterschiedlich einzuschätzen: zum einen wurden gewisse Punkte (Einbindung der Bundesländer) aufgegriffen, viele jedoch nicht (z.B. schlug das Programmkomitee die Schaffung eines Forschungsstättenkatalogs und die Prämierung der besten Projekte vor, beides flankierende Maßnahmen). Die 'nachhaltigste' Rolle erfüllte das Komitee in der Schaffung von Leitkriterien (siehe Tabelle 13); zu diesen Kriterien gehören etwa ,150 Projekte'.

\subsection{REGIONALE ANKNÜPFUNGSPUNKTE}

Eine Kooperation mit regionalen Akteuren herbeizuführen ist eines der Ziele der Initiative Mikrotechnik. Mit diesem Abschnitt wollen wir aufzeigen, welche regionalen Anknüpfungspunkte für eine Initiative Mikrotechnik möglich sind.

\section{Oberösterreich}

Im Regierungsübereinkommen der ÖVP OÖ bzw. den Grünen Oberösterreichs wird „Mikro“ als Schlüsseltechnologie bezeichnet: „Darüber hinaus streben wir die [...] Förderung von Schlüsseltechnologien (neue Werkstoffe, Bio, Mikro, Nano, Medizintechnik) in Fortsetzung der erfolgreichen Zusammenarbeit mit der J.K. Universität an.“20

\section{Tirol}

Die Tiroler Zukunftsstiftung ${ }^{21}$ definiert „Mikrotechnik“ als Bereich, der für die regionale Wirtschaft von Interesse sein wird und integriert ihn in ihren Mechatronik Bereich. Die

\footnotetext{
${ }^{19}$ Dem Evaluierungsteam lagen die Tagesordnungen und Protokolle der 1. Sitzung vom 16. Mai 2000, die Tagesordnung der 2. Sitzung am 30. November 2000 und schließlich Tagesordnung und Protokolle der 3. Sitzung vom 21. Dezember 2001 vor.

${ }^{20}$ ÖVP OÖ, Grüne Oberösterreich 2003

${ }^{21}$ Tiroler Zukunftsstiftung 2004, Seite 31f.
} 
Zukunftsstiftung fördert darüber hinaus das „Innsbrucker Nano Netzwerk“, das Tiroler Universitätsinstitute und Unternehmen in einem Konsortium vereinigt. Durch die westösterreichische Nano Initiative (w-INN) wird versucht, das bestehende Netzwerk auszubauen und wirtschaftsnahe Forschung im Bereich der Nanowissenschaften zu fördern. „Die Bündelung aller regionalen Aktivitäten steht im Vordergrund, um langfristig marktfähige Produkte, wissenschaftlichen Fortschritt und einen internationalen Wettbewerbsvorteil zu erlangen.“22

\section{Niederösterreich}

Im Rahmen seiner Technologieoffensive ${ }^{23}$ unterstützt Niederösterreich die Entwicklung von Mikrosystemtechnik und Nanotechnologie und misst ihr hohes Zukunftspotential zu.

\section{Vorarlberg}

Um die Entwicklung in Richtung Mikro- und Nanotechnologie in Vorarlberg zu unterstützen, trägt das Land Vorarlberg die Kosten des Aufbaus des Instituts für angewandte Mikrosystemtechnik (IAMT) an der Fachhochschule Vorarlberg ${ }^{24}$. Das Forschungszentrum soll der Wirtschaft für angewandte F\&E-Projekte zugänglich sein. Ziel ist der Zusammenschluss der in der Mikro- und Nanotechnologie tätigen Hochschulen der EUREGIO Bodensee ${ }^{25}$ zu einem „Mikro- und Nanotechnologie-Netzwerk“, um sich im Bereich der Forschung und Weiterbildung thematisch besser abstimmen zu können.

\section{Wien}

In ihrer Studie „Mikrosystemtechnik / Nanotechnologie“ vom Herbst 2002 führt die Magistratsabteilung 26 der Stadt Wien (heute: MA 27) die Bedeutung der Mikrosystemtechnik als Schlüsseltechnologie aus und entwickelt Konzepte, in welcher Weise Wien sich in diesem Bereich weiterentwickeln könnte. Jedoch hat weder der Wiener Wissenschafts- und Technologiefonds WWTF noch der Wiener Wirtschaftsförderungsfonds WWFF zur Zeit Ausschreibungen aus dem Bereich Mikro- oder Nanotechnologie geplant.

\section{Steiermark}

Die Steiermärkischen Landesregierung initiierte im Jahr 2001 das Nanotechnologienetzwerk NANONET-Styria mit dem Ziel, die Steiermark als Forschungs- und Wirtschaftsstandort zu fördern. Folgende Vision steht im Mittelpunkt.: „Die Steiermark soll in fünf Jahren (2007) eine national und international anerkannte Region im Bereich der Nanotechnologie sein. ${ }^{\text {"26 }}$ Die NANONET-Styria Initiative ist ,ein sehr positives Beispiel einer funktionierenden Abstimmung zwischen den verschiedenen Politikbereichen (Forschung, Wirtschaft, Bildung) sowie der Nutzung unterschiedlicher Finanzierungsquellen (EU-Regionalförderung, Zukunftsfonds Steiermark, andere regionale und nationale Quellen) ${ }^{6 \cdot 27}$.

Eine wichtige Weiterentwicklung stellt das Projekt Wissensbilanz dar, das die Entwicklung und Umsetzung eines Forschungsnetzwerkes erleichtern soll. „Mit der Wissensbilanz werden

\footnotetext{
${ }^{22}$ Dr. Vogl. Geschäftsführer von HITT (health information technologies tirol)

${ }^{23}$ Viehböck 2001

${ }^{24}$ www.fh-vorarlberg.ac.at

${ }^{25}$ Grenzregion von mehreren benachbarten Staaten, die somit als eigenständiges transnationales Gebilde gelten

${ }^{26}$ NANONET Styria, Positionspaper, Dezember 2002, Seite 2

${ }^{27}$ Forschungsstrategie Steiermark, Juni 2004, Seite 22
} 
die strategischen Ziele des NANONET-Styria mit den zur Verfügung stehenden immateriellen Potentialen und den Aktivitäten zur Entwicklung des Netzwerkes in Beziehung gesetzt. ‘28

Da der Bereich der Nanotechnologie eine Zukunftstechnologie des 21. Jahrhunderts darstellt, hat das Land Steiermark den konkreten Handlungsbedarf durch das Netzwerk NANONET Styria abgedeckt und wird als das ,aktivste und bestvorbereiteste Bundesland im Bereich der Nanotechnologie ${ }^{\text {“229 }}$ gesehen.

\section{Kärnten}

Um die strategische Neuausrichtung der CTR AG (Carinthian Tech Research) zu erleichtern, unterstützt das Land Kärnten verstärkt den Schwerpunkt Mikrotechnologie mit Hilfe von Förderungen. Die CTR AG und das deutsche Fraunhofer-Insitut arbeiten in diesem Bereich eng zusammen, um den Aufbau eines anwendungsorientierten Kompetenzzentrums für Mikrosystemtechnik zu verwirklichen. Der Lösungsansatz der CTR AG ist nicht die Entwicklung einzelner Komponenten in der Mikrosystemtechnik, ,sondern vielmehr deren Nutzen in unterschiedlichsten Kombinationen sowie deren sinnvolles Einfügen in bestehende Produkte. ${ }^{\text {‘30 }}$

\section{Salzburg}

Das Land Salzburg definiert seine Stärken in den Bereichen Dienstleistung, Tourismus, Kultur, Kommunikation, Landschaft / Naturraum, also nicht in klassischen High-TechBereichen. Initiativen in den 'Klassikern' der Hochtechnologie (also auch Mikro- und Nanotechnologie) werden als extrem ressourcenintensiv eingeschätzt, mit beträchtlicher nationaler und internationaler Konkurrenz. ${ }^{31}$

\subsection{WURDEN DIE RICHTIGEN FLANKIERENDEN MAßNAHMEN GESETZT?}

In seinem „Konzept als Diskussionsgrundlage“ schlägt C. Kukla („Um [...] dem generellen Ziel der Förderung der Mikrotechnik in Österreich gerecht zu werden, ist eine Fülle von Begleitmaßnahmen notwendig") die Einrichtung folgender flankierender Maßnahmen vor:

\section{Programmmanagement}

- FFF als „Anlaufstelle“ für Erstkontakte und Primärberatung einrichten

- Bildung von Schwerpunktbereichen entsprechend der Branchen

- Einbeziehung regionaler Einrichtungen (,train the trainer“)

\section{Forschung}

- Schaffung eines Forschungsstättenkatalogs

- Schaffung eines Unternehmenskatalogs (Datenbank potentieller forschender Firmen)

- Förderung reiner Forschungsprojekte mit einer Grundidee für Anwendung in Firmen

\footnotetext{
${ }^{28}$ Wissensbilanz NANONET Styria 2004

${ }^{29}$ Wissensbilanz NANONET Styria 2004

${ }^{30} \mathrm{http}: / / \mathrm{www} . \mathrm{ctr} . \mathrm{at} / \mathrm{de} / \mathrm{mikrosystemtechnik.asp}$

${ }^{31}$ Ohler et. Al, 2001
} 


\section{Technologietransfer}

- Awareness

- Klassische Werbung

- Vermittlung von Möglichkeiten der Mikrotechnik ${ }^{32}$

\section{Verbreiterung der Technologien - Mikrotechnik}

- Firmenprojekte zur Produkt- und Verfahrensentwicklung

- Feasibility Studien

- Maßnahmen zur Absicherung der Verwertung (Patente, Lizenzen, Verbrauchsmuster)

\section{Internationalisierung}

- Durch Kooperationen mit anderen europäischen Förderstellen und europäischen Programmen $^{33}$

In Box 4 werden nun diese vorgeschlagenen Maßnahmen dem tatsächlich während der Laufzeit gesetzten Aktivitäten gegenübergestellt.

Box 4: Flankierende Maßnahmen der „Initiative Mikrotechnik“

\begin{tabular}{|c|c|}
\hline Vorgeschlagene Maßnahme & Was ist tatsächlich passiert? \\
\hline $\begin{array}{l}\text { FFF als „Anlaufstelle“ für } \\
\text { Erstkontakte und } \\
\text { Primärberatung einrichten }\end{array}$ & $\begin{array}{l}\text { Der FFF ist insofern Anlaufstelle für Erstkontakte, als dass } \\
\text { er in seiner Tätigkeit insgesamt Förderberatung anbietet. } \\
\text { Primärberatung für Mikrotechnikprogramme, die sich von } \\
\text { anderen (bottom up) Projekten des FFF unterscheidet, gibt } \\
\text { es nicht. Die OGMS }{ }^{34} \text { wurde in Ansätzen als eine solche } \\
\text { Anlaufstelle genutzt. }\end{array}$ \\
\hline $\begin{array}{l}\text { Bildung von } \\
\text { Schwerpunktbereichen } \\
\text { entsprechend der Branchen }\end{array}$ & $\begin{array}{l}\text { Wurde nicht vorgenommen. Eine solche } \\
\text { Schwerpunktbildung ist aus Sicht der EvaluatorInnen auch } \\
\text { wenig empfehlenswert, braucht sie doch einen sehr hohen } \\
\text { Fachwissensbedarf im FFF und ist schwer auf Aktualität und } \\
\text { Treffsicherheit überprüfbar. Darüber hinaus hat das } \\
\text { Programmkomitee in seiner 1. Sitzung festgehalten, dass } \\
\text { „zumindest zu Beginn“ das Programm möglichst breit sein } \\
\text { soll. }{ }^{35}\end{array}$ \\
\hline $\begin{array}{l}\text { Einbeziehung regionaler } \\
\text { Einrichtungen (,train the } \\
\text { trainer“) }\end{array}$ & $\begin{array}{l}\text { Einbeziehung der FH Vorarlberg und der FH Wiener } \\
\text { Neustadt. Hier ist noch weiteres Potential in anderen } \\
\text { Bundesländern vorhanden. (Vgl. hierzu Abschnitt } 5.2 \\
\text { Regionale Anknüpfungspunkte)). Das Programmkomitee hat } \\
\text { darüber hinaus die Idee entwickelt, WIFI-BeraterInnen als } \\
\text { „MultiplikatorInnen“ einzusetzen, was in Oberösterreich } \\
\text { (TIM: Technologie- Innovations- Management, WIFI OÖ) } \\
\text { aufgegriffen wurde. }\end{array}$ \\
\hline $\begin{array}{l}\text { Schaffung eines } \\
\text { Forschungsstättenkatalogs }\end{array}$ & $\begin{array}{l}\text { Ein Forschungsstättenkatalog wurde nicht geschaffen. } \\
\text { Allerdings liegt mit der Publikation „Nano Technology and } \\
\text { Nano Science in Austria at a glance“ des BIT zumindest ein } \\
\text { Forschungsstättenkatalog für die Nanotechnologie vor. }\end{array}$ \\
\hline
\end{tabular}

\footnotetext{
${ }^{32}$ Beratung, Verwertung der Projektergebnisse, Tagungen, Seminare, Ausbildung, Schulungen

${ }^{33} \mathrm{zB}$ Eurimus im Rahmen von EUREKA

${ }^{34}$ Vgl. hierzu Protokoll zu dem Workshop Mikrosystemtechnik Österreich, vom 22. - 23. 5. 2001, Puchberg / Schneeberg

${ }^{35}$ Vgl. Protokoll der 1. Sitzung des Programmkomitees Mikrotechnik vom 16. Mai 2000
} 


\begin{tabular}{|c|c|}
\hline Vorgeschlagene Maßnahme & Was ist tatsächlich passiert? \\
\hline $\begin{array}{l}\text { Schaffung eines } \\
\text { Unternehmenskatalogs } \\
\text { (Datenbank potentieller } \\
\text { forschender Firmen) }\end{array}$ & $\begin{array}{l}\text { Ein solcher Katalog wurde nicht produziert. Gewisse } \\
\text { Elemente werden von der oben zitierten BIT-Publikation } \\
\text { abgedeckt; andererseits wird in der Nano-Initiative und in } \\
\text { anderen Bemühungen den Unternehmen (und den } \\
\text { Forschenden) eine Plattform für Kontaktpflege und - } \\
\text { knüpfung geboten. }\end{array}$ \\
\hline $\begin{array}{l}\text { Förderung reiner } \\
\text { Forschungsprojekte mit einer } \\
\text { Grundidee für Anwendung in } \\
\text { Firmen }\end{array}$ & $\begin{array}{l}\text { Eine solche Förderung wurde nicht vorgenommen. Mit der } \\
\text { Translational Research - Schiene des FWF, den } \\
\text { Verbundprojekten der Nano-Initiative und den Targeted } \\
\text { Basic Research Projekten des FFF gibt es allerdings gleich } \\
\text { drei jüngst etablierte Fördermöglichkeiten in diesem } \\
\text { Bereich. }\end{array}$ \\
\hline Awareness & $\begin{array}{l}\text { Im Bereich der Awareness- Maßnahmen sind vor allem } \\
2001 \text { einige Schritte gesetzt worden: Veranstaltungen bei } \\
\text { Joanneum Research in Weiz, TIM (Linz), ein Workshop } \\
\text { Puchberg und einige Veranstaltungen der OGMS; } 2002 \\
\text { schließlich eine „Roadshow Mikro- und } \\
\text { Nanotechnologie“636, veranstaltet von FFF, OGMS, und } \\
\text { Nanonet Styria. 2003 wurde auf weitere Maßnahmen } \\
\text { verzichtet. }\end{array}$ \\
\hline Klassische Werbung & $\begin{array}{l}\text { Klassische Werbung erfolgte über die Imagefolder des FFF } \\
\text { zur Initiative sowie über die Homepage des Fonds. }\end{array}$ \\
\hline Beratung allgemein & $\begin{array}{l}\text { Es wurde keine Bratung, die über die herkömmliche } \\
\text { Beratung von FFF-bottom-up-Projekten hinausgeht, } \\
\text { vorgesehen. }\end{array}$ \\
\hline $\begin{array}{l}\text { Beratung zur Verwertung von } \\
\text { Projektergebnissen }\end{array}$ & $\begin{array}{l}\text { Es wurde keine Bratung, die über die herkömmliche } \\
\text { Beratung von FFF-bottom-up-Projekten hinausgeht, } \\
\text { vorgesehen. }\end{array}$ \\
\hline Tagungen & $\begin{array}{l}\text { Es wurden keine (wissenschaftlichen) Tagungen gefördert. } \\
\text { Am 12. Juni } 2002 \text { wurde allerdings eine Podiumsdiskussion } \\
\text { zum Thema im Rahmen der Wiener Neustädter } \\
\text { Technologiegespräche durchgeführt. }\end{array}$ \\
\hline Seminare & $\begin{array}{l}\text { Es wurden keine (wissenschaftlichen) Seminare gefördert. In } \\
\text { der Wirtschaftskammer Österreich war ein Seminar } \\
\text { (,Mikrotechnik revolutioniert Produkte und Märkte“) für } \\
\text { KMU anberaumt worden, das dann mangels } \\
\text { TeilnehmerInnen abgesagt werden musste. }\end{array}$ \\
\hline Ausbildung, Schulung & $\begin{array}{l}\text { Der FFF übernahm oder förderte in der Initiative } \\
\text { Mikrotechnik keine Art von Schulungen bzw. } \\
\text { Schulungskosten. Weiters wurden in der vom FFF zur } \\
\text { Verfügung gestellten Datenbank keine Projekte der FFF- } \\
\text { Sonderaktion „Nachwuchsförderung“ bekannt, die der } \\
\text { Initiative Mikrotechnik zuzuordnen wären. }\end{array}$ \\
\hline $\begin{array}{l}\text { Firmenprojekte zur Produkt- } \\
\text { und Verfahrensentwicklung }\end{array}$ & $\begin{array}{l}\text { Die Mehrzahl der Projekte der Initiative Mikrotechnik waren } \\
\text { angewandte Forschungsprojekte zur Produkt- und }\end{array}$ \\
\hline
\end{tabular}

${ }^{36}$ TFZ Technologieforschungszentrum Wiener Neustadt, 12.6. 2002, W.E.I.Z. Weizer Energie Innovationszentrum 13. 6. 2002, CATT Central Austrian Technology Transfer and Training, 18. 6. 2002, Wirtschaftskammer Innsbruck 19.6. 2002, Fachhochschule Dornbirn, 20. 6. 2002 


\begin{tabular}{|ll|}
\hline \multicolumn{3}{|c|}{ Worgeschlagene Maßnahme } & \multicolumn{3}{|c|}{ Verfahrensentwicklung. } \\
\hline Feasibility Studien & $\begin{array}{l}\text { Von den (uns vorliegenden) 158 Projekten aus der } \\
\text { Aktionslinie Feasibility Studies hatten 3 Studien } \\
\text { Mikrotechnik - Inhalte. }\end{array}$ \\
\hline $\begin{array}{l}\text { Maßnahmen zur Absicherung } \\
\text { der Verwertung (Patente, } \\
\text { Lizenzen, Verbrauchsmuster) }\end{array}$ & Hier wurden keine Maßnahmen gesetzt. \\
\hline $\begin{array}{l}\text { Kooperationen mit anderen } \\
\text { europäischen Förderstellen } \\
\text { und europäischen } \\
\text { Programmen }\end{array}$ & $\begin{array}{l}\text { Der FFF ist Koordinator eines ERA-Nets im Bereich der } \\
\text { Mikrotechnologie und hat somit die Basis für Kooperationen } \\
\text { in diesem Bereich hergestellt. }\end{array}$ \\
\hline
\end{tabular}

\section{Wurden begleitend zur Initiative andere Aktionslinien des FFF genutzt?}

Neben der Initiative Mikrotechnik unterhält der FFF verschiedene Aktionslinien. Feasibility Studies (mit dem Ziel externe Machbarkeitsstudien, die von KMUs in Auftrag gegeben werden durchzuführen), F\&E Dynamik (mit dem Ziel die betriebliche Infrastruktur österreichischer KMUs nachhaltig zu verbessern), die Lebensmittelinitiative, Nachwuchsförderung (mit dem Ziel Kooperation und Kommunikation zwischen Wissenschaft und Wirtschaft zu fördern, indem junge ForscherInnen vor dem Studienabschluss gemeinsam mit ihrem/ihrer BetreuerIn und einem Unternehmen ein Forschungsprojekt als Diplomarbeit oder Dissertation realisieren), EUREKA, EU Anbahnungsförderung, e-content und Start up (mit dem Ziel technologieorientierte, junge Unternehmen zu fördern).

Im Programmfolder des FFF wurde insbesondere auf die Vernetzung der verschiedenen Förderinstrumente bzw. Aktionslinien des FFF aufmerksam gemacht. Insbesondere die Aktionslinien Feasibility und F\&E Dynamik, zu denen Joanneum Research auch Zugang zu den relevanten Förderdaten hatte, stellen gute Möglichkeiten dar um kleinen und mittleren Unternehmen den Zugang zu Anwendungs- und Forschungsmöglichkeiten im Bereich der Mikrotechnik zu ermöglichen. Auch die Aktionslinie Nachwuchsforschung scheint als Awareness Maßnahme für Unternehmen geeignet, um einerseits Kontakte zu forschenden Institutionen aufzubauen und andererseits Nutzungsmöglichkeiten der Mikrotechnologie zu identifizieren und in die Unternehmungsplanung zu integrieren.

Die Datenanalyse zu den begleitenden Sonderaktionen für die Aktionslinien F\&E Dynamik und Feasibility die in Kapitel 3.2.7 erfolgt zeigt jedoch, dass die Unternehmen die vorgeschlagenen Maßnahmen nur sehr eingeschränkt nutzten. Im gesamten Förderzeitraum der Initiative Mikrotechnik wurden im Rahmen der Mikrotechnik Initiative 3 Feasibility Studies von insgesamt 158 Feasibilty Studies beantragt. Von den 36 F\&E Dynamik Projekten im Beobachtungszeitraum 2001-2003 wurde keines der Initiative Mikrotechnik zugerechnet.

\section{Fazit:}

Nicht alle der vorgeschlagenen Maßnahmen wurden also aufgegriffen: Hier ist nachdrücklich darauf hinzuweisen, dass dieses "Nichtaufgreifen" selbst nicht als Kritik am Maßnahmendesign aufgefasst werden darf. Es ist das gute Recht einer jeden 
Fördereinrichtung, Vorschläge die im Rahmen einer ex ante Evaluierung oder einer feasibility zu einem neuen Programm gemacht werden, nicht nur aufzunehmen, sondern auch (begründet) zu verwerfen. Jedenfalls wurden in der Initiative Mikrotechnik keine Schwerpunktbereiche gebildet. Der FFF ist nicht als Anlaufstelle für Primäreinreicher des Feldes Mikrotechnik eingerichtet worden, noch wurde ein Forschungsstättenkatalog oder ein Katalog der im Feld der Mikrotechnik forschenden Unternehmen abgefasst. Reine Forschungsprojekte wurden vom FFF nicht gefördert, ebenso keine Aktionen zur besseren Verwertung von Projektergebnissen oder Maßnahmen zur Absicherung der Verwertung gestartet.

Unserer Einschätzung nach argumentiert der FFF, dass durch die Kombination der Initiative Mikrotechnik mit seinen FFF-Aktionslinien (insbesondere Feasibility Studies und Nachwuchsförderung, aber auch F\&E Dynamik) ohnehin eine Vielzahl der von Kukla geforderten flankierenden Maßnahmen abgedeckt ist. Nicht in jedem Programm, in jeder Initiative das Rad neu erfinden zu wollen, ist begrüßenswert und effizient. Nun ist aber festzustellen, dass Feasibility Studies und F\&E Dynamik von MikrotechnikProjekten kaum bzw. gar nicht genutzt werden. Hier scheint es einer vermehrten Anstrengung seitens des FFF zu bedürfen, um das wechselseitige Nutzbarmachen der unterschiedlichen Aktionslinien zu gewährleisten: mehr Beratung, mehr Hinweisen auf andere Aktionslinien, mehr Awareness.

\subsection{SIND DIE GEWÄHLTEN FÖRDERINSTRUMENTE DAZU GEEIGNET, DIE SELBST GESETZTEN ZIELE ZU ERREICHEN?}

In der Logic Chart Analyse weiter oben wurde die Ziel- und Maßnahmenebene der Initiative zusammengefasst und beschrieben. In weiterer Folge geben wir eine Einschätzung auf Ebene der verschiedenen Ziele, in wie weit die gewählten Instrumente zu deren Erreichung geeignet waren.

\section{Erreichung kritischer Massen}

Der Wunsch, mit einem Programm kritische Massen zu erreichen, stellt uns vor das Problem, definieren zu müssen, was sind „kritische Massen“ für das „Technologiefeld Mikro- und Nanotechnologie“ im österreichischen Kontext. Wir nehmen an, dass mit „kritischen Massen nicht die Forschungskapazität der österreichischen Universitäten und außeruniversitären Einrichtungen gemeint ist, sondern die Mikrotechnikkapazitäten in Unternehmen (sei es als Nachfrager, sei es als Anwender). Wir wollen nun diese Definition umschiffen, und versuchen wie folgt zu antworten: Setzt sich ein Programm das Ziel, „kritische Massen“ erzeugen $\mathrm{zu}$ wollen, so steht $\mathrm{zu}$ Beginn des Programms der Befund, das zu wenige Unternehmen in dem Bereich Mikrotechnik tätig sind bzw. dass Unternehmen im Bereich zu wenig forschen. Wir unterstellen also, dass hier das Ziel gesetzt wurde, das mehr Unternehmen im Bereich der Mikrotechnologie forschen bzw. dass Unternehmen in diesem Bereich mehr Forschungs- und Entwicklungsarbeit leisten sollen.

Der FFF leistet hier seinen Beitrag, indem er in seinen Aktionslinien ein klares Signal an die Unternehmen setzt, im Bereich der Mikrotechnik zu forschen. Der FFF hat sich bemüht, auf 
neue Unternehmen zuzugehen, in dem er Awarenessmaßnahmen förderte und eine Roadshow veranstaltete. Weitere Diffusions- oder Verbreitungsmaßnahmen, wie z.B. Informationsplattformen, Wissenstransfer (firm to firm oder über Köpfe), Personaltransfer (InnovationsassistentInnen, Post Docs oder ähnliches), Bereitstellung von BeraterInnen, oder Unterstützung im Trainingsbereich setzte der FFF nicht.

\section{Fazit:}

An und für sich ist ein eingeführtes, bekanntes Förderinstrument wie die FFF Projekte ein probates Mittel, um Unternehmen für Forschung und Entwicklung zu motivieren. Eine Flankierung mit Beratung und Awarenessmaßnahmen wäre eine sinnvolle Ergänzung.

\section{Kooperation Wissenschaft Wirtschaft}

Es gibt ein weites Feld von Maßnahmen, die die Stärkung der Kooperationsbeziehungen von Wissenschaft und Wirtschaft zum Inhalt haben - in Österreich reicht das von Personaltransferprogrammen wie dem Post-Doc Programm des FWF, über thematische Programme (FIT-IT) bis hin zu den Kompetenzzentrenprogrammen (Kind, Knet, Kplus) des Bundes.

\section{Fazit:}

Dass die Standard-Projektförderung des FFF (die schließlich die bedeutendste Forschungsförderung für österreichische Unternehmen ist) in diesem Spektrum eine besondere Rolle einnehmen würde, wäre weit hergeholt. Zwar sieht der FFF für Kooperationsprojekte höhere Barwerte vor, er trägt dies aber nicht nach aussen und regt somit nicht zu mehr Wissenschaft - Wirtschaft - Kooperationen bei. Wir weisen darauf hin, dass - im Gegensatz zu anderen thematischen Programmen wie FIT IT, die auch mit Beteiligung des FFF abgewickelt werden und eine Kooperationsverpflichtung aufweisen - es in der Initiative Mikrotechnik keine kooperationsfördernde Auflagen gibt.

\section{Einbindung regionaler Player}

Der FFF hat versucht, über die gemeinsame Gestaltung von Veranstaltungen und die Einbindung regionaler Player in das Programmkomitee die Initiative Mikrotechnik auf eine breite Basis zu stellen.

\section{Fazit:}

Wir glauben, dass der vom FFF gewählte Weg, regionale Akteure in seine Aktionslinie einzubinden, der richtige ist. Der in anderen Programmen (Kplus, AplusB) vom Bund gewählte Weg, Landesregierungen bzw. deren Agenturen als Mitfinanciées in die Programme einzubinden, hat zwar für den Bund budgetäre Vorteile, hat aber zu einer Irritation mancher Länder geführt ${ }^{37}$ ). Diese besitzen aufgrund ihrer zum Teil erheblichen finanziellen Bindungen aus diesen Programmen keinen Gestaltungsraum mehr, eine eigenständige Forschungs- und Technologiepolitik zu machen. Die Taktik des FFF -

\footnotetext{
${ }^{37}$ Hier sieht man sich als Land in einer „Zwickmühle“: Eigentlich muss man ja nur dann der Finanzierung eines Zentrums zustimmen, wenn man das auch will; im Aufsichtsrat der als Zentren gestalteten GmbHs hat man seinem Finanzierungsteil entsprechende Mitspracherechte. Gleichzeitig sieht man sich aber einem gewissen (auch sozialen) Druck ausgesetzt, jedenfalls diese Chancen aufzugreifen, die die Entsehung eines Zentrums für ein Land und eine Region bedeuten.
} 
regionale Plattformen nutzen, um zu Unternehmen aus den Bundesländern zu kommen und Akteure im Rahmen des Programmkomitees so weit wie möglich einzubinden ist richtig.

\section{Signalwirkung an die Technologiepolitik}

Wie entsteht Technologiepolitik in Österreich? Zum einen spielt klarerweise die sogenannten stakeholder des Politikprozesses (also öffentliche Verwaltung; Fonds, Rat etc.) in der Planung von Politiken eine entscheidende Rolle. Gleichzeitig entsteht Politik entlang bestimmter „patterns of behaviour": Grießler ${ }^{38}$ beschreibt diese Muster als ein österreichisches „Technologiepolitik-Spiel“, in dem ein innerer Kreis, der aus wenigen Personen besteht und regelmäßig im Rahmen von Sitzungen, Beratungsgremien oder Aufsichtsräten trifft, entlang von Kontaktsystemen in zunehmender Komplexität agiert, in dem neue Ideen oder neue Konzepte der Einsatz sind, bei dem Versuch, Technologiepolitik zu dominieren. Welche Initiativen neu entstehen und welche nicht, ist Ergebnis dieses Spiels.

\section{Fazit:}

Die Lancierung einer „Initiative Mikrotechnik“ ist sicherlich ein probates Mittel, um sich in diesem Spiel zu positionieren, sozusagen „Claims abzustecken“ und Zeichen zu setzen.

\section{Internationaler Technologietransfer}

Im Bereich des Zieles „Internationaler Technologietransfer“" werden unterschiedliche Aspekte adressiert. Einerseits die Positionierung des Fonds im internationalen Netz der Forschungsförderer, andererseits um Unternehmen (etwa in Vorarlberg) dazu zu bringen, grenzübergreifende Forschungskooperationen einzugehen.

\section{Fazit:}

Der FFF hat die Initiative Mikrotechnik perfekt genutzt, sich im Netzwerk der europäischen Forschungseinrichtungen (und somit, wenn man so will, im europäischen Forschungsraum) zu positionieren (MNT ERA Net) ${ }^{39}$. Dieses ERA Net soll in Zukunft auch Ausgangspunkt für Kooperationen auf Unternehmens- und Forschungseinrichtungsebene sein.

\subsection{WELCHE SCHLÜSSE AUS DER FFF EVALUIERUNG FÜR DIE INITIATIVE MIKROTECHNIK GEZOGEN WERDEN KÖNNEN}

Die Evaluierung des FFF weist darauf hin, dass seit 2000 der Anteil an FFF-Projekten, die im Rahmen von Initiativen und Programmen gefördert wurden, gestiegen ist. Mit der Abwicklung von ITF-Schwerpunkten ist die Bedeutung der Technologieprogramme auch in

\footnotetext{
${ }^{38}$ Erich Grießler: Innovation und Politikgestaltung: Administrative Kulturen in der Technologiepolitik. Ein Vergleich zwischen Österreich und den Niederlanden. In: Rupert Pichler (Hg.): Innovationsmuster in der österreichischen Wirtschaftsgeschichte. StudienVerlag, Innsbruck 2003

${ }^{39}$ Ziel des ERA-NETs ist es, "to coordinate the currently fragmented research and promotion programmes and exchange good practice on aspects of programme design and managements." Man will aber auch "trans border partnerships between business and research centers" fördern. Spiegelt man diesen Anspruch am Budget des ERA-NET von 2,4 Mio $€$ und der Anzahl der beteiligten Länder und Einrichtungen (Deutschland, Norwegen, Niederlande, Irland, Spanien, Schweden, Schweiz) so wird klar, dass aus dem ERA NET höchstens Impulse in diese Richtung ausgehen können.
} 
Österreich gestiegen. Damit und mit der Setzung eigener Initiativen hat der FFF in gewisser Weise ein pro-aktives Zeichen gesetzt. Dennoch kommt die Evaluierung zu dem Schluss: ,The role of the FFF in this development was to some extent ambiguous. On the one hand many observers have seen FFF as fairly reluctant to take up top-down programme activites. On the other hand FFF has built up a degree of programme management competence and actually provides programme management services for several technology programmes, competence networks (Knet) and industrial competence centers (kind) on a contract basis.' (Arnold et al. p. 36).

Prima vista ist der FFF mit der Setzung eigener Programmschwerpunkten einer der Hauptkritikpunkte der Evaluierung, i.e. eine ,reaktive' Strategie zu verfolgen und keine adäquaten Förderinstrumente entwickelt zu haben, zuvorgekommen. Neben der beauftragen Programmabwicklung hat der FFF auf eigene Initiative hin Programme (,Aktionslinien') entwickelt. Dennoch bleibt das Spezifische an Förderprogrammen - abgesehen von der Installierung eines Programmmanagements, das sich auch neben dem Alltagsgeschäft um Begleitmaßnahmen, Marketing etc. zu kümmern hat - verglichen mit der bottom-up Förderschiene zu unscharf. Die Schnittstelle wird zu wenig klar definiert. Projekte aus den Programmen werden genauso behandelt wie aus der bottom-up Förderung und es entsteht mitunter der Eindruck, dass Projekte aus den thematischen Aktionslinien eine Teilmenge aus den FFF-Projekten darstellen. Hier lassen sich aus der FFF - Evaluierung wichtige Schlüsse für die Formulierung zukünftiger Programme ziehen:

1. eine Balance zwischen top-down und bottom-up Strategien

2. ein stärkeres Programmmanagement

3. die Entwicklung neuer Instrumente

4. Entwicklung integrativer, d.h. verschiedene Technolgien, Sektoren, Disziplinen umfassender, Ansätze

5. eine Verstärkung des Netzwerkgedankens

6. eine deutliche Verstärkung der Internationalisierung der Förderwerber 


\section{Effektivität - oder..., ,was hat der FFF bei wem erreicht?"}

Fragen nach Effektivität und Effizienz sind im Rahmen einer Zwischenevaluierung einer Fördermaßnahme der industriellen Forschung nur im geringen Ausmaß beantwortbar. Effekte brauchen in der Regel Zeit und der Erfolg oder Misserfolg eines Projektes ist auch nicht unmittelbar nach Projektende feststellbar. Diese Frage sollte daher in extenso in einer eventuellen ex-post Evaluierung behandelt werden. Wir versuchen dennoch einige Aspekte dieser Fragestellungen - Outputs, Charakter der Projekte, Orientierung etc. - zu beantworten.

\subsection{WAS SIND DIE KONKRETEN OUTPUTS DER INITIATIVE?}

Auf der Seite der konkreten Outputs der Initiative Mikrotechnik stehen 110 geförderte Projekte, die von 65 Unternehmen und 7 Arbeitsgemeinschaften durchgeführt werden.

\subsection{WIE IST DER CHARAKTER DER GEFÖRDERTEN PROJEKTE?}

Die geförderten Projekte der Initiative Mikrotechnik unterscheiden sich in mehrerer Hinsicht von der Gesamtheit der geförderten Projekte des FFF:

Erstens sind die Projektkosten in der Initiative deutlich höher als die der Gesamtheit der FFF Förderwerber. Zweitens unterscheiden sich die durchführenden Unternehmen deutlich von der Grundgesamtheit der FFF-Förderwerber: Während für Klein- und Mittelbetriebe insgesamt rund $80 \%$ der geförderten Betriebe darstellen, die rund $50 \%$ der Fördermittel zugesprochen bekommen (vgl. FFF Jahresbericht 2002), liegt der Anteil der KMU in der Initiative Mikrotechnik lediglich bei $63 \%$, die mit 25,6\% der zugesprochenen Fördermittel deutlich weniger Mittel lukrieren als größere Unternehmen.

Drittens sind die Antragsteller der Initiative Mikrotechnik deutlich aktivere Förderwerber als die Gesamtheit der Antragsteller des FFF.

\subsection{NEU- ODER STAMMKUNDEN?}

Wie in Kapitel 3.2.2 „Neue Antragsteller \& Frequenz der Förderanträge“ dargestellt, haben lediglich 9 Unternehmen ausschließlich einen Antrag in der Initiative Mikrotechnik gestellt, und sich nicht auch für andere FFF-Projekte im Zeitraum 1995-2003 beworben. Im Durchschnitt haben sich die Unternehmen der Initiative für 18 zusätzliche Projekte beworben, während jedes Unternehmen im Schnitt 2,8 Förderanträge gestellt hat.

Die OGMS, die als Akquisiteur neuer Kunden eingeplant war, hatte Schwierigkeiten, ihre in Sie gesetzten Erwartungen zu erfüllen: die Projektantragsstellung gestaltete sich langwieriger als ursprünglich angenommen. ${ }^{40} \mathrm{Ob}$ über die Roadshow oder über das Betreiben der OGMS

${ }^{40}$ Zeilinger, Kukla 2002 
neue Förderwerber des FFF gewonnen werden konnten, ist anhand der Projektdaten nicht nachvollziehbar.

\subsection{GRUNDLAGENORIENTIERT ODER ANGEWANDTE FORSCHUNG?}

Die Initiative Mikrotechnik hat sich zum Ziel gesetzt, auf Projektebene vor allem zur Anwendung und Entwicklung von neuen Produkten und Verfahren beizutragen. Der FFF, als Forschungsförderungsfonds der gewerblichen Wirtschaft, legt also per definitionem den Fokus der Forschungsförderung auf die angewandte, eher vermarktungsfähige Forschung. Die Verstärkung der Zusammenarbeit zwischen Wissenschaft und Wirtschaft ist aber ein zentrales Anliegen des FFF (vgl. Jahresbericht FFF 2002, p. 28), und der FFF versucht auch über Projekte der Initiative „Nachwuchsförderung“ (Förderung von Dissertationen und Diplomarbeiten im Dreiecksverhältnis Betrieb-wissenschaftliche Betreuung-StudentInnen), Kontakte zwischen Universitäten und Unternehmen zu unterstützen.

Anhand der Projektdaten des FFF ist es aber nicht möglich, den Grad der angewandten Forschung vs. der Grundlagenorientierung der Forschung, zB mittels eines Time to Market Indikators, zu erfassen. Dementsprechend kann über das Spannungsverhältnis Grundlagenforschung versus angewandte Forschung in der Initiative Mikrotechnik keine Aussage getroffen werden. Die Anzahl der Wissenschaft-Wirtschaft Kooperationen blieb in der Initiative Mikrotechnik allerdings unter dem vom Programmkomitee erhofften Zielbereich und über die Initiative Nachwuchsförderung lagen keine Informationen vor.

\subsection{ERFOLGSFAKTOREN DER INITIATIVE}

Eine der Aufgaben des Programmkomitees der Initiative Mikrotechnik war es, klare Erfolgsfaktoren für eine Zwischenevaluierung aufzustellen. Auch seitens des FFF gab es zu diesen Erfolgsfaktoren ein Bekenntnis - so werden diese in der Einladung zur 2. Sitzung des Programmkomitees durch den Geschäftsführer des Fonds, Herbert Wotke explizit angesprochen. ${ }^{41}$ Im März 2002 (d.h. mehr al ein Jahr nach Programmstart) wurde jeweils ein Wertebereich angegeben ${ }^{42}$, von dem vom Programmkomitee erwartet wurde, dass die untere Grenze eine realistische, die obere eine ambitionierte Grenze darstelle.

Tabelle 13: Leitkriterien der Initiative Mikrotechnik - Definition Programmkomitee

\begin{tabular}{|l|l|l|}
\hline \multicolumn{1}{|c|}{ Leitkriterium } & \multicolumn{1}{c|}{ Zielbereich } & \multicolumn{1}{c|}{ Tatsächlicher Wert } \\
\hline Anzahl der Projekte & $100-150$ & 150 beantragt, 110 bewilligt in 3 Jahren \\
\hline $\begin{array}{l}\text { Anzahl der antragsstellenden } \\
\text { Firmen }\end{array}$ & $50-70$ & 65 Unternehmen und 7 ARGEs \\
\hline Anzahl der Neuantragssteller & $>20 \%$ & $\begin{array}{l}14 \% \text { oder 9 Unternehmen mit } \\
\text { ausschließlichem Antrag in Mikrotechnik }\end{array}$ \\
\hline
\end{tabular}

\footnotetext{
${ }^{41}$ Tagesordnungspunkt 2: „Festlegen der Erfolgsfaktoren, die auch evaluiert werden, aabgeleitet aus dem Programm.“ Einladung Programmkomitee 2. Sitzung vom 19. Oktober 2000

${ }^{42}$ Vgl hiezu Zeilinger, Kukla 2002
} 


\begin{tabular}{|c|c|c|}
\hline Leitkriterium & Zielbereich & Tatsächlicher Wert \\
\hline & & 19\% inklusive 5 ARGEs \\
\hline $\begin{array}{l}\text { Kooperationen zwischen } \\
\text { Firmen } \\
\text { (davon international) }\end{array}$ & $>10 \%$ & $\begin{array}{l}6 \% \text { ARGEs } \\
\text { Internationale Kooperationen werden nicht } \\
\text { erfasst. }\end{array}$ \\
\hline $\begin{array}{l}\text { Kooperationen mit FH, } \\
\text { Hochschule } \\
\text { (davon international) }\end{array}$ & $>30 \%$ & $\begin{array}{l}27 \% \text { der durchgeführten Projekte } \\
\text { Internationale Kooperationen werden nicht } \\
\text { erfasst. }\end{array}$ \\
\hline $\begin{array}{l}\text { Grenzüberschreitende } \\
\text { Kooperationen }\end{array}$ & $>20 \%$ & werden nicht erfasst \\
\hline $\begin{array}{l}\text { Anteil der Projekte mit hoher } \\
\text { technologischer Qualität }\end{array}$ & $>60 \%$ & $\begin{array}{l}\text { Es wurde keine Schwellenwert definiert. Je } \\
\text { nach Schwellenwert 37\% bzw. } 63 \% \\
\text { Projekte mit hoher technologische r } \\
\text { Qualität }\end{array}$ \\
\hline $\begin{array}{l}\text { Projekte mit } \\
\text { Patentschutzstrategie }\end{array}$ & $30-40 \%$ & $\begin{array}{l}48 \% \text { haben konkrete Überlegungen oder } \\
\text { Schritte für ein Patent gemacht. } \\
\text { Zusätzlich ist bei rund } 13 \% \text { der } \\
\text { Antragsteller ein Patent vorhanden, } \\
\text { eingereicht oder erteilt worden. }\end{array}$ \\
\hline
\end{tabular}

Quelle: Zeilinger, Kukla 2002, eigene Darstellung

Es zeigt sich, dass die vom Programmkomitee angestrebten Zielkriterien - mit einigen Ausnahmen - erreicht wurden. Zu zwei der Leiterkriterien kann aufgrund der Datenerfassung des FFF keine Aussage getroffen werden.

Bereits im Frühjahr 2002 bemerkte der FFF, dass die Akquisition der OGMS von Unternehmen für die Projektantragstellung sich langwieriger gestaltet als anfangs angenommen und weniger Projekte als angekündigt eingereicht wurden. Nun verbesserte sich die Antragstellung in den Jahren 2002 und 2003 gegenüber dem ersten Jahr der Initiative aber erheblich. Was die Anzahl an Kooperationen von Unternehmen mit dem Wissenschaftssektor und auch internationale Kooperationen betrifft, so greifen die Datenerfassung des FFF und die Kriterien eindeutig zu kurz um positive Wirkungen auf Wirtschaft und Gesellschaft abzuleiten.

Ein weitere Zielsetzung aus dem Jahr 2002 war eine räumliche: Es zeigte sich damals, dass im Umfeld von Forschungseinrichtungen - also rund um die FH Wiener Neustadt, um Joanneum Weiz, die Universität Graz und FH Vorarlberg - regionale Häufungen waren. Betrachtet man die Verteilung von Projektanträgen und Unternehmen auf die österreichischen Bundesländer, so sind alle Bundesländer in der Initiative vertreten, wenngleich eine Vielzahl an Projektanträgen aus der Steiermark kommt. Dass es zu regionalen Häufungen der Förderwerber rund um Ballungszentren und im Umfeld von Forschungseinrichtungen kommt ist nachvollziehbar und nicht problematisch, da sich Unternehmen gemäß ihrem Tätigkeitsfeld eben auch verstärkt in regionaler Nähe zu Forschungszentren etablieren. 


\subsection{ZIELERREICHUNG}

Im Rahmen der Logic Chart Analyse wurden Mission und Ziele der Initiative Mikrotechnik identifiziert. Wir wollen die dort entwickelte Struktur zum Anlass nehmen, die Frage nach der „Zielerreichung“ in Subfragen zu zerlegen und versuchen, jeweils Antworten zu finden:

\section{Wurde das Technologiefeld „Mikrotechnik“bzw „Nanotechnik“ in Österreich durch den FFF verankert und weiterentwickelt?}

Insgesamt wickelte der FFF 110 Projekte im Feld „Mikro- und Nanotechnik“ mit einem Fördervolumen von rund 55 Millionen Euro ab. Zieht man die Ergebnisse der FFF Evaluierung zu Rate, so ergab diese unter anderem, dass „FFF's regular customer spend about $€ 1,40$ on $R \& D$ for every Euro they receive in subsidy. ${ }^{\text {“43 }}$ Zieht man des weiteren die Ergebnisse der im Zuge der zitierten Evaluierung vorgenommenen Befragung heran, so gibt über die Hälfte der FörderempfängerInnen an, dass FFF Projekte auf Produktinnovationen abzielen. 2/3 der Befragten beabsichtigten damit, neue Märkte zu erschließen. In Abwesenheit einer eigenen Befragung der Mikrotechnikprojekte ist dies der einzige Hinweis, den wir zur Beantwortung der hier aufgeworfenen Frage geben können. Leider fehlen auch Studien, die die gesamte Größe des „Mikrotechnik-Marktes“ für Österreich einschätzen. So ist die Antwort eine zurückhaltende:

\section{Fazit:}

Ja, der FFF hat seinen Beitrag zur Verankerung und Weiterentwicklung geleistet, allerdings können wir seine Bedeutung nicht seriös einschätzen.

\section{Geschah dies auf breiter Basis?}

Seit dem 3 jährigen Bestsehen der Initiative Mikrotechnik reichten 62 Unternehmen insgesamt 150 Projekte ein. Damit konnten die in den Leitkriterien angestrebten Werte für förderwerbende Unternehmen und Projekte erreicht werden. Die Zahl der Neuantragsteller blieb mit 9 Unternehmen (14\%) unter den Erwartungen. Was die Verteilung der Unternehmen auf Größenklassen an MitarbeiterInnen betrifft, so können 40 Unternehmen oder 63\% aufgrund ihrer MitarbeiterInnenzahl von weniger als 250 der Gruppe der kleinen und mittleren Unternehmen zugerechnet werden, die übrigen 23 Unternehmen waren groß.

\section{Fazit:}

Ja, die angestrebte Zahl an Förderwerber wurde erreicht. Konterkarriert wird dieses Bild von der geringen Anzahl von neuen Förderwerbern und dem starken Gewicht der großen Unternehmen im Projektportfolio.

In der regionalen Verteilung der Förderprojekte spielt die Steiermark mit 62 von 139 (exklusive der 9 Anträge von ARGEs und zweier Anträge von Unternehmen, die nicht in der Datenbank waren) beantragten Projekten eine dominante Rolle. Wien und Niederösterreich folgen mit 21 beantragten Projekten, von Kärntner Unternehmen stammen 19 Projekte. Tirol und Vorarlberg gemeinsam sowie Salzburg verzeichnen je 12 Projektanträge, Oberösterreich 10 und das Burgenland 3.

\footnotetext{
${ }^{43}$ Schibany et al., 2004
} 


\section{Wurden insbesondere KMU angesprochen?}

Von den 63 Unternehmen die Förderanträge in der Initiative Mikrotechnik gestellt haben, können 40 Unternehmen, also rund 63\%, der Gruppe der kleinen und mittleren Unternehmen zugerechnet werden. Zieht man die Anzahl der Förderbewilligungen in der Initiative Mikrotechnik als Maß für die Einbindung der kleinen und mittleren Unternehmen an der Initiative Mikrotechnik heran, so sinkt die Beteiligung der KMU an der Initiative auf $41 \%$.

Wird zusätzlich nicht nur der Unternehmensanteil, sondern auch die Verteilung der Projektmittel als Maß für das Augenmerk oder die Hauptzielgruppe der Initiative berücksichtigt, so verlagert sich der Fokus der Initiative auf große Unternehmen: Große Unternehmen mit mehr als 250 MitarbeiterInnen lukrierten 74,4\% der Fördermittel, während auf kleinere und mittlere Unternehmen 25,6\% der Fördermittel entfielen.

Laut dem Jahresbericht des FFF 2002 sind 80\% aller Antragssteller KMUs; $50 \%$ der Fördermittel fließen in kleine und mittlere Unternehmen.

Daraus ergibt sich unser Urteil:

Nein, in der Initiative Mikrotechnik wurden nicht überwiegend KMU angesprochen. Der FFF hat jedoch gezielt Großunternehmen zu einer Teilnahme an der Initiative Mikrotechnik geraten.

\section{Wurden vorhandene Technologien in Firmen gebracht und weiterentwickelt?}

Wir können über dieses Ziel nicht Auskunft geben. Einerseits sind wir keine Mikro- und NanoexpertInnen die die Technologien bewerten können, andererseits geben die Daten der Projektdatei keine Auskunft über Technologietransfer etc.

Fazit:

Diese Frage muss unbeantwortet bleiben.

\section{Wurden kritische Massen erreicht?}

Weiter oben versuchen wir diese Frage wie folgt zu umschreiben: Wir unterstellen, dass hier das Ziel gesetzt wurde, dass mehr Unternehmen im Bereich der Mikrotechnologie forschen bzw. das Unternehmen im Bereich mehr forschen und entwickeln. Wir definieren als „Newcomer“ jene Unternehmen, die nur einmal einen FFF Antrag im Rahmen der Initiatve gestellt haben $(14 \%$ der Antragssteller) und vergleichen dies mit dem gesamten Projektportfolio des FFF (über 50\% der Antragssteller seit 1995 haben nur einen Förderantrag an den FFF gestellt)

\section{Fazit:}

Nein, auf Grund der niedrigen Zahl der Neukunden, lediglich 9 Unternehmen haben sich ausschließlich für Projekte innerhalb der Initiative Mikrotechnik beworben, können wir nicht davon ausgehen, dass die Initiative Mikrotechnik einen Beitrag dazu geleistet hat, dass kritische Massen entstehen.

Nein, denn aufgrund der hohen Fördervolumen für große Player wurden keine zusätzlichen Kunden angesprochen, sondern ohnehin starke Unternehmen, die in ihren Kernkompetenzen agieren, gestärkt (siehe Verteilung der Mittel). 
Konterkariert wird dieses Bild von folgender Überlegung: Auf Grund der hohen Volumen des Programms (über 48 Millionen Euro) und der Ergebnisse der FFFEvaluierung können wir andererseits davon ausgehen, dass der FFF dazu beigetragen hat, dass Österreichische Unternehmen im Bereich Mikro- und Nanotechnologie mehr forschen.

\section{Wurde zur Kooperation „Wissenschaft - Wirtschaft“ beigetragen?}

Von den 110 bewilligten Projekten werden rund 73\% ohne Wissenschaft - Wirtschaft Kooperation durchgeführt und 27\% mit Kooperationen. Damit konnte der in den Leitkriterien angepeilte Wert von 30\% knapp nicht erreicht werden.

Hinsichtlich der Unternehmensgröße ist die kooperationsfreundlichste Gruppe jene der mittelgroßen Unternehmen mit 100 bis 250 Beschäftigten, die für rund $21 \%$ der durchgeführten Projekte verantwortlich zeichnet.

Betrachtet man die Projektgröße, so sind es eher kleiner Projekte mit einem Projektvolumen von $100.000-250.000 €$, die den höchsten Anteil an Kooperationsprojekten mit Forschungseinrichtungen und Universitäten ausmachen.

Für die Initiative Mikrotechnik bedeutet das, dass vor allem innovative mittelständische Unternehmen die über nicht genügen ,in house“ Forschungskapazitäten verfügen, auf externe Forschungskapazitäten zurückgreifen.

Fazit:

Aufgrund des Fördermechanismus, der keine expliziten Anreize für Kooperationen bietet, und der nicht signifikanten Änderung der Ablehnungsrate zwischen Projekten „,mit“ und „ohne“ Kooperation, kann keine Aussage getroffen werden ob zusätzlich zur Kooperation zwischen Wissenschaft und Wirtschaft beigetragen wurde.

\section{Wurden regionale Player eingebunden? Kam es zu einer Bündelung der Aktivitäten der Länder unter dem Dach der Initiative Mikrotechnik?}

Mit der FH Vorarlberg, der FH Wiener Neustadt, dem CATT oder der Joanneum Research wurden (in unterschiedlichen Zusammenhängen und zu unterschiedlichen Zeitpunkten) auf die Leistungen regionaler Player zurückgegriffen. Wie Abschnitt 5.3 oben zeigt, gibt es hier Dynamik und Erweiterungspotential. Von einer Bündelung dieser Aktivitäten ist allerdings nicht auszugehen.

Fazit:

Ja, es wurden regionale Player eingebunden. Zu einer Bündelung der Länderaktivitäten kam es nicht.

\section{Konnte der FFF mit der Initiative Mikrotechnik sich in Österreichs Technologiepolitik positionieren?}

Mit Mikrotechnik hat der FFF 2001 eine neuen thematischen Schwerpunkt gesetzt. Seit dem hat der Rat Mikro- und Nanotechnologie auf die nationale Agenda gesetzt; man könnte nun argumentieren, dass der FFF mit seiner Initiative einen Beitrag zur nationalen Sichtbarkeit geleistet und mitgeholfen hat, auf die Bedeutung dieser Basistechnologie aufmerksam zu 
machen. Als Mißererfolg ist zu werten, dass das bmvit in weiterer Folge sich entschlossen hat, die Nano - Initiative über die ASA und nicht vorrangig über den FFF abzuwickeln. Allerdings sind die Autoren nicht in der Lage, die Motive die zu diesem Schritt führten, nachzuvollziehen und abzuschätzen, in wie weit für den FFF diese Entscheidung überhaupt beeinflussbar war.

\section{Kam es zu einem internationalen Technologietransfer?}

Aufgrund der vorhandenen Datenlage können wir keine Aussagen darüber treffen, ob es zu einem internationalen Technologietransfer gekommen ist, also zu Projekten mit ausländischer Beteiligung.

\section{Mitnahmeeffekte und Additionalität}

Die Messung der Additionalität eines Programes ist eine der herausforderndsten Aufgaben in der Evaluierung von Forschungs- und Technologiepolitik. Schibany et al. haben dies - auf Projektebene - für den FFF versucht und diese Herausforderung als folgende Fragen skizziert:

- Input additionality: Do public contributions to private research boost total private R\&D expenditures - and if so, do they boost them by an amount which is larger than the amount of taxpayer's money which was used in this way?

- Output additionality: What is the effect of the subsidies research on a firm's turnover, profit, etc.?

- Behavioural additionality: In how far does the existence and availability of public subsidies alter firm's research decisions? (Schibany et al, Seite 28)

Da es sich bei der Evaluierung der Initiative Mikrotechnik um eine Zwischenevaluierung und keine ex-post Evaluierung handelt, kann die Frage nach der Input und Output Additionalität der Initiative unserer Ansicht nach noch nicht schlüssig beantwortet werden. Aufgrund der hohen Konzentration einiger weniger sehr aktiver Förderwerber verglichen mit der Gesamtheit der FFF-Förderwerber in der Initiative Mikrotechnik, ist diese Frage für eine expost Evaluierung der Initiative allerdings von großem Belang.

Darüber hinaus stellt sich nun die Frage nach der Additionalität des Programms: Wäre auf Projektebene nicht ohnehin im Bereich der Mikrotechnik etwas passiert? Was wurde durch die Themensetzung des FFF zusätzlich ausgelöst?

Bei Programmen wie den Kompetenzzentrumsprogramm Kplus ist die Beantwortung dieser Frage relativ einfach: Kplus ist qua seiner Existenz additional - ohne öffentliche Förderung hätten sich diese Partner zu dieser Zeit nicht zusammengefunden, um in diesem Ausmaß und unter einem gemeinsamen Gesellschaftsdach zu kooperieren.

Bei der Initiative Mikrotechnik ist eine so einfache Argumentationskette nicht möglich. Aus einem Interview mit dem FFF wissen wir, dass nicht alle Projekte, die jetzt als Mikrotechnikprojekte ausgewiesen werden, unter diesem Titel beim FFF eingereicht wurden, 
sondern dass auch herkömmliche bottom up Projekte von den FFF-GutachterInnen der Initiative zugeordnet wurden. ${ }^{44}$

Darüber hinaus hat die Initiative Mikrotechnik keine zusätzlichen Budgetmittel, die Projekte werden aus dem Regelbudget des FFF finanziert. Somit kann man nicht argumentieren, dass durch die Initiative Mikrotechnik mehr F\&E in Österreich als ohnehin passiert wäre höchstens andere.

Passierte ,andere F\&E“? Eine inhaltliche Antwort ist auf Basis der zur Verfügung stehenden Daten nicht möglich. Jedenfalls können wir jedoch untersuchen, ob „Förderroutinees“ oder Neukunden TeilnehmerInnen an der Initiative waren, also, ob andere Unternehmen F\&E Projekte abgewickelt haben: Von den 63 förderwerbenden Unternehmen stellten 9 Unternehmen lediglich Anträge in der Initiative Mikrotechnik. Die Gesamtheit der Antragsteller der Initiative Mikrotechnik hat sich im Durchschnitt im Zeitraum 1995-2003 für 18 (Median 5) weitere Projektförderungen beworben, während der Durchschnitt der Gesamtheit der Förderwerber sich für 2,8 Projekte beworben hat (vergleiche detaillierte Darstellung in Kapitel 3.2.2). Die Unternehmen der Initiative Mikrotechnik können daher als sehr aktive Förderwerber beschrieben werden, sie sind deutlich aktivere Förderwerber als die Gesamtheit der Antragsteller, so genannte Förderroutinees.

\section{Fazit}

Wir glauben, dass die Initiative Mikrotechnik - auf Grund des Design des Programms (keine zusätzlichen Budgetmittel, Zuordnung von Projekten) nur wenig additionale Effekte ausgelöst hat. Auch die Ausführungen in Bezug auf die flankierenden Maßnahmen unterstreichen das. Für die Projekte sind ähnliche Aussagen wie bei der FFF/FWF Evaluierung zu treffen.

\footnotetext{
${ }^{44}$ Eine Quantifizierung ist laut Auskunft FFF nicht möglich.
} 


\section{Handlungsoptionen}

Am Beginn einer Reflexion über Handlungsoptionen muss sich der FFF über grundsätzlicheres klar werden:

- Will der FFF auch zukünftig Schwerpunkte setzen?

- Und wenn ja, in welcher Form und in welchem Themenbereich?

Als Orientierungspunkte für eine solche Diskussion mögen die Ergebnisse der FFF Evaluierung dienen. Für die Formulierung zukünftiger Programme wird dort vorgeschlagen:

- eine Balance zwischen top-down und bottom-up Strategien

- ein stärkeres Programmmanagement

- die Entwicklung neuer Instrumente

- Entwicklung integrativer, d.h. verschiedene Technolgien, Sektoren, Disziplinen umfassender Ansätze

- eine Verstärkung des Netzwerkgedankens

- eine deutliche Verstärkung der Internationalisierung der Förderwerber

Wir haben die weiter unten folgenden Handlungsoptionen unter der folgenden Annahme entwickelt, dass der FFF diese Empfehlungen (zumindest zum Teil) aufgreift, auch hinkünftig Schwerpunkte setzen will und dies in Form von „Initiativen“, „Aktionslinien“, „Programmen“ tut. ${ }^{45}$. Die Entscheidungsgremien des FFF (bzw. der FFG) sollen sich ein Bild schaffen, welche Schwerpunkte sie hinkünftig verfolgen wollen; der Rat für Forschungs- und Technologieentwicklung hat hier Orientierungspunkte gesetzt. Aus Sicht der Evaluatoren gibt es jedenfalls keine Gründe, einen Schwerpunkt Mikrotechnik nicht fortzuführen.

\section{Die Initiative Mikrotechnik in der neuen FFG}

In der 22. Gesetzgebungsperiode des Nationalrates wurde die Errichtung einer Österreichischen Forschungsförderungsgesellschaft, kurz FFG, beschlossen. In diese Gesellschaft wurde mit September 2004, neben der ASA, der TIG und dem BIT, auch die Forschungsförderungsfonds für die gewerbliche Wirtschaft übertragen.

$\mathrm{Zu}$ den Aufgaben der FGG gehört unter anderem:

- Förderung von Forschungs- und Entwicklungsvorhaben

- Durchführung strategischer Fördermaßnahmen und -programme,

- die Förderung der Kooperation von Wissenschaft und Wirtschaft.

Des weiteren wird im Gesetzestext festgehalten, dass ,die Gesellschaft übernimmt im Zuge der Gesamtrechtsnachfolge auch die [...] bestehenden Richtlinien [...]. Die auf Grund bestehender Richtlinien der übertragenen Einrichtungen durchgeführten Maßnahmen sind zu

\footnotetext{
${ }^{45}$ Wir verwenden diese Begriffe als Synonyme.
} 
übernehmen und fortzuführen“. ${ }^{46}$ Dies bedeutet, dass die Grundlage für die Fortführung der „Initiative Mikrotechnik“ durch den FFF bzw. durch die FFG gegeben ist. Diese hat Arbeitsprogramme auf mehrjähriger Basis zu erstellen, die von der Geschäftsführung der FFG erarbeitet werden, durch deren Aufsichtsrat beschlossen, und schließlich von bmvit und BMWA genehmigt. ${ }^{47}$

\subsection{OPTION 1: FORTFÜHRUNG IN DER BISHERIGEN FORM}

Eine „Initiative“ des FFF sollte prinzipiell so gestaltet sein, dass sich der Aufwand, der damit verbunden ist, auch lohnt (bzw. lohnen kann); ein bloßes Abwickeln ,herkömmlicher' FFFbottom up Projekte unter einem Etikett macht keinen Sinn und schafft keine Additionalität.

Wir haben in diesem Bericht einige Punkte festgehalten, die wir als wesentliche Kritikpunkte an der Initiative Mikrotechnik verstanden wissen wollen. Diese Kritikpunkte sind

- Die Intiative hat einige der selbst gesteckten Ziele nicht erreicht

- Der FFF spricht mit der Initiative vorrangig Förderprofis an, nicht KMUs oder Unternehmen die sich neu mit Mikrotechnik auseinandersetzen.

- Die Initiative schöpft die Möglichkeiten von flankierenden Maßnahmen nicht aus.

- Das Management der Initiative hat nicht genügend Ressourcen zur Verfügung, um (durchaus komplexe) Abwicklungs- und Vernetzungsschritte oder Öffentlichkeitsarbeiten zu setzen.

Die Initiative Mikrotechnik ist nicht bloß ein Etikett für eine Teilmenge von FFF Projekten es gibt einen Programmmanager, flankierende Maßnahmen, verbindliche Ziele. Allerdings glauben wir, dass das Potential zur Programmausrichtung bei weitem nicht ausgeschöpft ist und die Schnittstelle zwischen der bottom up Förderschiene des FFF und den Spezifika eines Technologieprogramms noch zu wenig klar erkennbar ist .

Wir glauben, dass es die bisher gezeigten Ergebnisse der Initiative es nicht rechtfertigen, sie in ihrer bisherigen Form fortzusetzen.

\subsection{OPTION 2: EINSTELLEN DER INITIATIVE MIKROTECHNIK}

Eine Option „Einstellen der Initiative Mikrotechnik“ bedeutete nicht, dass der FFF sich entscheidet, nicht mehr im Bereich Mikro- und Nanotechnologie zu fördern. Schließlich ist es Unternehmen unbenommen, auch weiterhin mikro- und nanotechnische Projekte beim FFF im Rahmen der bottom-up Projektförderung einzureichen. Das was eingestellt werden könnte, ist das Hervorheben von Mikrotechnik als besondere Aktionslinie des FFF: also das, was die Initiative zur Initiative macht (die Positionierung von R. Zeilinger als „Mister Mikrotechnologie“, Awareness, Öffentlichkeitsarbeit etc.)

So stellt sich die Frage, mit welcher Signalwirkung ein solches Einstellen verbunden wäre: Ein Einstellen wäre sicherlich mit einem „Aufschrei“ der KundInnen des FFF (und der Mikro- und Nanoszene) verbunden und es müsste offensiv kommuniziert werden, dass ein

${ }^{46}$ FFG-G $\S 4(4)$ 
Ende der Initiative Mikrotechnik nicht ein Ende der Projektförderung im Bereich bedeuten würde. Außerdem würde ein solches Signal (,der FFF nimmt Mikro und Nano von der forschungs- und technologiepolitischen Agenda") zum jetzigen Zeitpunkt eine Konterkarierung der österreichischen Anstrengungen im Technologiebereich sein. ${ }^{48}$.

Ein „nicht so wie bisher fortführen“ ist nicht automatisch mit einer Empfehlung gleichzusetzten, generell die Initiative Mikrotechnik aufzugeben. Wir führen weiter oben aus, dass der FFF mit „Mikrotechnik“ einen richtigen Ansatz gewählt hat.

Des weiteren glauben wir, dass die Initiative Mikrotechnik Potential hat, die Nano Initiative zu ergänzen und ein Beibehalten des Schwerpunktes Mikrotechnik ein Kontinuitätssignal an Unternehmen und ForscherInnen darstellt.

Wir glauben, dass die Schwerpunktsetzung Mikrotechnik beibehalten werden sollte.

\subsection{OPTION 3:MODIFIZIERTE FORTFÜHRUNG.}

Unser Vorschlag an den FFF ist es, zwischen der Option 3, „Reformieren“, und der Option 4, „Zusammenführen“, zu wählen. Wir schlagen dem FFF vor, folgende Veränderungen im Design des Programmes Mikrotechnik zu prüfen:

- Weg von der Initiative, hin zum Programm: Wir regen an, dass der FFF Aktionslinien nicht als Zusammenfassung von bottom up Projekten versteht, sondern als eigenständige Programme mit begrenzter Laufzeit, mit Management, und verstärkter Öffentlichkeitsarbeit.

- Wording: Der FFF unterstützt Mikro- und Nanoprojekte und sollte diese Tatsache auch nachdrücklich transparent machen, zB. indem er den Namen des Programms anpasst.

- Gute Ansatzpunkte bewahren: Mit der angedachten Verschränkung mit anderen Aktionslinien (insbesondere Feasability Studies) setzt der FFF ein wohltuendes Zeichen, dass nicht für jedes Programm das Rad neu erfunden werden muss. Allerdings zeigt die schwache Nutzung von solchen Verschränkungen bisher, dass ein verstärktes Bewusstmachen der hier innewohnenden Möglichkeiten notwendig ist. Auch ist es notwendig, dass sich der FFF so wie bisher aktiv in die Leitungsgremien der Nano-Initiative einbringt.

- Programmmanagement: Verstärkte Öffentlichkeitsarbeit, mehr Awareness und mehr Koordination zu anderen Aktionslinien bedeutet mehr Management-Aufwand. Es darf nicht nur Ansprüche an ein Programm Mikrotechnik geben, es müssen auch Ressourcen finanzieller und personeller Natur freigemacht werden.

- Überprüfung des Ziels KMU: Der FFF möge überprüfen, inwieweit ein Herausstellen der KMU in den Zielsetzungen der Initiative sinnvoll ist.

\footnotetext{
${ }^{47}$ Ebenda, $\S 8$

${ }^{48}$ Einen Aufschrei einer Klientel wird es immer geben, wenn ein Programm oder ein Schwerpunkt eingestellt wird. Dies wird von EntscheidungsträgerInnen ab und an hinzunehmen sein, ansonsten würde das österreichische Maßnahmenportfolio auf immer festgeschrieben sein. Abzuwiegen bleibt, ob es begründete Einwände gibt oder der Aufschrei ausschließlich eigennutzorientiert ist.
} 
- Leitkriterien: Auch hinkünftig sollen Leitkriterien Verwendung finden, allerdings sollten diese im Monitoring System Berücksichtigung finden, insbesondere das Themenfeld Kooperationen.

- Anpassen des Instrumentes „Projekt“: Der FFF soll prüfen, in wie weit er für das Programm Mikrotechnik sein Instrument der Projektförderung anpassen kann. Als Orientierungspunkte können hierbei die Erfahrungen mit FIT-IT, aber auch die Erfahrungen aus dem ERA-NET herangezogen werden. Aus Sicht der EvaluatorInnen wären folgende Modifikationen notwendig:

o Anreizsysteme für Kooperationsprojekte unter Leitung von Forschungseinrichtungen.

o Anreizsysteme für KMU, sich an der Initiative Mikrotechnik zu beteiligen. ${ }^{49}$

- Nutzen des neuen Instrumentes „Brückenschlagprojekte“: Mit Projekten aus der Programmfamile „tranlational research“ und „targeted basic research“ stehen neue Instrumente zur Verfügung, um Ergebnisse der Grundlagenforschung der Wirtschaft näherzubringen. Der FFF sollte mit seiner Initative der Brückenschlagprojekte aktiv um Projekte aus dem Bereich der Mikro- und Nanotechnologie werben.

- Flankierende Maßnahmen, die auf Inventions- und Absorptionsfähigkeit der künftigen abzielen: Ein mehr an Vorträgen, Round Tables, Train the Trainer als bisher wäre wünschenswert.

- Abstimmung mit weiteren Förderprogrammen: Der FFF soll gemeinsam mit dem Management anderer Programme prüfen ${ }^{50}$, ob durch eine weiterführende Zusammenarbeit Synergieeffekte lukriert werden können. Als Beispiel hierfür sollen gelten:

o FIT - IT fördert langfristige Kooperationsprojekte im Bereich IKT und tut dies im Rahmen von Schwerpunkten. Eine Denkvariante wäre, dass - nach sorgfältiger Prüfung des Themas - einer dieser Schwerpunkte von FIT - IT aus dem Bereich Mikro - und Nanotechnologie kommen könnte (etwa „Telecommunication Engineering" $)^{51}$

o protec fördert in seinen verschiedenen Ausformungen Technologietransferprojekte. Eine Möglichkeit bestünde darin, einen kleineren call dieses Programmes für Mikro- und Nanotechnik zu earmarken und so zu vermehrten Diffusionsaktivitäten beizutragen.

- Nutzung bestehender Netze und Initiativen: Wir glauben, dass es unter UnternehmerInnen und WissenschafterInnen auch so etwas wie eine Netzwerkmüdigkeit gibt. Wir schlagen daher explizit nicht vor, ein neues „MikroNet Austria“ zu starten, sondern wir regen an, bestehende Netze oder Vereine für Awareness und Öffentlichkeitsarbeit zu nutzen. Wichtig ist es hierbei, dass sich das

\footnotetext{
${ }^{49}$ Analog könnte auch von unabhängiger Stelle überprüft werden, ob die Zielgruppe KMU nun für Mikro und Nanotechnologie- Projekte überhaupt eine zutreffende ist (d.h. ob Forschung im bereich der Mikro und Nanotechnologie in Österreich einfach in größeren Unternehmen stattfindet und es keine entsprechende KMULandschaft gibt) oder ob es anderer Instrumente (Personaltransfer, Information etc.) als Projekte bedarf, um KMU für Mikro- und Nanotechnologie zu motivieren.

${ }^{50}$ Inhalte dieser Prüfung müssen sein: Passt ein solches Ansinnen in die jeweilige Förderlogik? Ist eine Schwerpunktsetzung nach den jeweiligen Kriterien der Programme gerechtfertigt?

${ }^{51}$ Dies ist als Beispiel und nicht als Vorschlag zu werten, genau diesen Schwerpunkt aufzugreifen.
} 
Programm Mikrotechnik nicht auf eine/n AkteurIn stützt, sondern sich möglichst vieler unterschiedlicher AkteurInnen bedient.

- Aufgreifen weiterer regionaler Anknüpfungspunkte. Wir zeigen verschiedene regionale Entwicklungen auf, die als Ansatzpunkt für Awarenessmaßnahmen und Multiplikatoren-Effekte gewählt werden können.

- Spiegeln an den Erkenntnissen des ERA-NET: Die Erfahrungen aus dem MNT ERANET sollen in die Neuausrichtung einbezogen werden.

\subsection{OPTION 4: ZUSAMMENFÜHRUNG MIT DER NANO INITIATIVE ÖSTERREICH}

Es ist für die Evaluatoren nicht abschätzbar, in wie weit eine Zusammenführung von Programmlinien aus verschiedenen Agenturen bzw. Fonds eine realistische Denkvariante auch in der FFG - ist. Sollte der FFF einer solchen Lösung zuneigen, möchten wir einige Punkte aufzeigen. Wir empfehlen dem FFF jedenfalls, diese Option nur unter gewissen Bedingungen anzudenken: dass nämlich dem FFF eine entsprechende Rolle in der Leitung des Programmes und in der Gestaltung der Initiative eingeräumt wird. Insbesondere sollte überprüft werden, in wie weit die Aktionslinie feasibility studies durch die Nano Initiative dupliziert wird. Auch würde ein Zusammenführen nicht nur vom FFF verlangen, einen Schritt auf die ASA zuzugehen, auch umgekehrt muss Bereitschaft vorhanden sein, bestehende Bilder und Abläufe zu überdenken und gegebenenfalls zu modifizieren.

Wir glauben, dass FFF-Projekte eine wichtige Ergänzung zu dem Förderinstrumentarium sind, dass die Nano Initiative darstellen. Es wird mit dieser Option vom FFF explizit nicht eingefordert, dass Design der Verbundprojekte oder die Begutachtungspraxis der ASA zu übernehmen. Jedenfalls können folgende Punkte Gegenstand einer Zusammenführung sein

- Ein gemeinsames Dach Mikro \& Nano: Gemeinsame Öffentlichkeitsarbeit, gemeinsamer Webauftritt, gemeinsame Anlaufstelle für Beratung, gemeinsame Awarenessveranstaltungen, Informationsschienen etc.

- Die Nano Initiative für Mikrotechnik „aufmachen“: Uns wurde in verschiedenen Gesprächen versichert, dass der Übergang von Mikrotechnologie zur Nanotechnologie ein fließender ist. Es spricht daher aus unserer Sicht wenig dagegen, anzudenken, das Instrumentarium der Nano - Initiative für Mikrotechnik aufzumachen. Auch eine Differenzierung zwischen „Calls“ wäre denkbar.

- Wording: Es muss - auch im Titel der Initiative - ein klares Signal gesetzt werden, dass nicht nur Nano- sondern auch Mikrotechnologie förderwürdig ist.

- Mikrotechnologie darf kein Thema zweiter Ordnung werden: Es muss sichergestellt sein, dass die Mikrotechnologie in keinen Förderwettbewerb mit NanotechnikProjekten gerät und in der Auswahl.

- Bereitschaft, voneinander zu lernen: Die Nano - Initiative hat ihre Förderroutinen eben erst aufgebaut, der FFF hat jahrelange Erfahrung in Begutachtung und 
Abwicklung: Aus beiden Gesichtspunkten kann man neues für Maßnahmendesign und-abwicklung einbringen.

- Nutzen des neuen Instrumentes „Brückenschlagprojekte“: (siehe weiter oben)

- Flankierende Maßnahmen, die auf Inventions- und Absorptionsfähigkeit der künftigen abzielen: (siehe weiter oben)

- Abstimmung mit weiteren Förderprogrammen: (siehe weiter oben)

Der FFF soll - gemeinsam mit der ASA und innerhalb der FFG - in einen Diskussionsprozess treten, der ausloten soll, ob es die möglichen Synergieeffekte einer Zusammenlegung rechtfertigen, in einen aufwendigen Prozess der Annäherung zu treten und ein gerade entstandenes Programm einem Redesign zu unterziehen.

\subsection{EINE MISCHFORM AUS OPTION 3 UND 4}

Abschließend möchten wir auf die Möglichkeit für die EntscheidungsträgerInnen innerhalb der FFG hinweisen, die Handlungsoptionen 3 und $4 \mathrm{zu}$ kombinieren und quasi „,cherry picking" vorzunehmen. Vorstellbar wäre beispielsweise, ein gemeinsames Dach für die beiden Programme vorzusehen, jedoch die Abwicklungsmodalitäten (v.a. Begutachtung) und den inhaltlichen Focus unterschiedlich zu belassen und dies auch als „Asset“ herauszuarbeiten. 


\section{Anhang}

\section{Literatur}

Arnold, E., et. al. Evaluation of the Austrian Industrial Research Promotion Fund (FFF) and the Austrian Science Fund (FWF). Synthesis Report. Vienna 2004

Bundesministerium für Wissenschaft und Verkehr: Grünbuch zur österreichischen Forschungspolitik, Wien 1999

Büro für internationale Forschungs- und Technologiekooperationen : Nano Technology and Nano Science in Austria at a glance. Wien, Oktober 2002

Dachs, B., et al.: Zukunftspotentiale der österreichischen Forschung. Studie im Auftrag des Rats für Forschung und Technologieentwicklung, ARC systems research, Joanneum Research, Wien 2003

Ecmc Europäisches Zentrum für Medienkompetenz GmbH: Mikrosystemtechnik (MST) Strukturen eines Kompetenzfeldes, Expertise für die GIB NRW GmbH, März 2004

ERA-Net Informationsblatt: Coordination Action; Huge Potential for Tiny Technology“, Brüssel 2003

ERA-NET: DRAFT Frequently Asked Questions, $1^{\text {st }}$ June 2004

Europäische Kommission, Investing in Research: an Action Plan for Europe, Communication from the European Commission, EUR 20804 EN COM(2003) 226 final, 2003

Europäische Kommission, Raising EU R\&D Intensity: Improving the Effectiveness of Public Support Mechanisms for Private Sector Research and Development: Direct Measures, Report to the European Commission from an Independent Expert Group, 2003

Europäische Kommission, Raising EU R\&D Intensity: Improving the Effectiveness of the Mix of Public Support Mechanisms for Private Sector Research and Development, Report to the European Commission from an Independent Expert Group, 2003

Glenck, Emmanuel: The Austrian Nano Initiative: Instruments of the National RTD Policy for Nanoscience and Nanotechnology, Vienna 2003 (?)

Grießler, Erich: Innovation und Politikgestaltung: Administrative Kulturen in der Technologiepolitik. Ein Vergleich zwischen Österreich und den Niederlanden. In: Rupert Pichler (Hg.): Innovationsmuster in der österreichischen Wirtschaftsgeschichte, Studienverlag 2003

Kukla, C.: Mikrotechnik in Österreich, Konzept als Diskussionsgrundlage, 1. 12. 2000

Mahlich, J., Ohler, F., Puwein, W., Gamsjäger, C.: Evaluierung der Sonderaktion Holzforschung, Technopolis, Wifo, im Auftrag des Forschungsförderungsfonds für die gewerbliche Wirtschaft (FFF), November 2002

Ohler, F. (Technopolis Austria), Sturn, D., Gruber, M., Ploder, M. (Joanneum Research): Wissenschafts- und Forschungsleitbild des Bundeslandes Salzburg, Salzburg 2001

ÖVP ÖÖ, Grüne Oberösterreichs: Zukunft Oberösterreich 2003 - 2009, Arbeitsübereinkommen der Oberösterreichischen Volkspartei und der Grünen Oberösterreichs,Linz, 23. Oktober 2003

Prognos AG, Deutsche Bank, Hochschule für Bankwirtschaft, Technopolis Group: Evaluation des Förderkonzepts Mikrosystemtechnik 2000+. Im Auftrag des 
Bundesministeriums für Bildung und Forschung, Berlin, Basel, Frankfurt, Amsterdam, Wien, November 2002

Rat für Forschung und Technologieentwicklung, Nationaler Forschungs- und Innovationsplan, Dezember 2002

Rat für Forschung und Technologieentwicklung: Forschungsstrategie Austria „2,5\% + plus“ Wohlstand durch Forschung und Innovation. Wien 2001 B

Rat für Forschung und Technologieentwicklung: Nationaler Forschungs- und Innovationsplan, Rat für Forschung und Technologieentwicklung, Wien, Dezember 2002

Rat für Forschung und Technologieentwicklung: Vision 2005 Durch Innovation zu den Besten. Wien 2001 A

Tiroler Zukunftsstiftung: Tätigkeitsbereich 2003. Inssbruck 2004

Viehböck, DI Franz: Technologiekonzept Niederösterreich, St. Pölten 2001

W.K. Kellogg Foundation, Logic Model Development Guide. Using Logic Models to Bring together Planning, Evaluation \& Action, W.K. Kellogg Foundation, Michigan 2001

Wurm, Christian, Unger, Elisabeth: Mikrosystemtechnik / Nanotechnologie. Bedeutung für den Wirtschaftsstandort Wien, Wien 2002

Zeilinger, Kukla, C.: Zwischenbericht zur Initiative Mikrotechnik Österreich für das Jahr 2001, Wien 2002 
Tabelle 14: Dokumente, Homepages, Sonstige Quellen

Austrian Space Agency, ASA http://www.asaspace.at/

\begin{tabular}{ll}
\hline BIT & $\underline{\text { http://www.bit.ac.at/ }}$ \\
\hline BBT. Bundesamt für & $\underline{\text { http://www.bbt.admin.ch/ }}$ \\
Berufsbildung und & \\
Technologie &
\end{tabular}

CLUES. Competence Land- http://www.key-to-innovation.org/ scapes Unfolding Europe's

Strengths

\begin{tabular}{|c|c|}
\hline ERA Net - FAQs & ftp://ftp.cordis.lu/pub/coordination/docs/faq_0406_en.pdf \\
\hline FFF & $\underline{\text { www.fff.co.at }}$ \\
\hline MST Online & http://www.mstonline.de/ \\
\hline ÖVP Oberösterreich & $\begin{array}{l}\text { www.ooevp.at/opencms/opencms/OEVP/downloads/2003/z } \\
\text { ukunft-ooe-2003-2009.pdf }\end{array}$ \\
\hline $\begin{array}{l}\text { Rat für Forschung und } \\
\text { Technologieentwicklung }\end{array}$ & $\underline{w w w . r a t-f t e . a t}$ \\
\hline $\begin{array}{l}\text { Technologieoffensive } \\
\text { Niederösterreich }\end{array}$ & http://www.tecnetarea.at/ \\
\hline TOP NANO 21 Initiative & www.temas.ch/nano/nano_homepage.nsf \\
\hline $\begin{array}{l}\text { WWTF Wiener Wissenschafts- } \\
\text { und Technologiefonds }\end{array}$ & $\underline{\text { http://www.wwtf.at }}$ \\
\hline $\begin{array}{l}\text { WWFF Wiener } \\
\text { Wirtschaftsförderungsfonds }\end{array}$ & http://www.wwff.at \\
\hline Regierungsprogramme & http://www.austria.gv.at/ \\
\hline WK Kellogg Foundation & http://www.wkkf.org/ \\
\hline
\end{tabular}


Tabelle 15: Programme und Initiativen des FFF 1994-2002

\begin{tabular}{|c|c|c|c|c|c|c|c|c|c|c|}
\hline mandated programmes & own initiatives & 94 & 95 & 96 & 97 & 98 & 99 & 00 & 01 & 02 \\
\hline ITF & & $\mathrm{x}$ & $\mathrm{x}$ & $\mathrm{x}$ & $\mathrm{x}$ & $\mathrm{x}$ & $\mathrm{x}$ & $\mathrm{X}$ & $\mathrm{x}$ & $\mathrm{x}$ \\
\hline ÖNB (since 1982) & & $\mathrm{x}$ & $\mathrm{x}$ & $\mathrm{x}$ & $\mathrm{x}$ & $\mathrm{x}$ & $\mathrm{x}$ & $\mathrm{x}$ & $\mathrm{x}$ & $\mathrm{x}$ \\
\hline Siedlungswasserwirtschaft & & & $\mathrm{x}$ & $\mathrm{x}$ & $\mathrm{x}$ & $\mathrm{x}$ & $\mathrm{x}$ & $\mathrm{x}$ & & \\
\hline Impulsförderung (ACR) & & & $\mathrm{x}$ & $\mathrm{x}$ & $\mathrm{x}$ & $\mathrm{x}$ & & & & \\
\hline Wachstumsförderung (ACR) & & & & & & & $\mathrm{x}$ & $\mathrm{X}$ & $\mathrm{x}$ & $\mathrm{x}$ \\
\hline Impulsförderung FHS-Wirtschaft & & & & & $\mathrm{x}$ & $\mathrm{x}$ & $\mathrm{x}$ & $\mathrm{x}$ & $\mathrm{x}$ & \\
\hline $\begin{array}{l}\text { Impulsprogramm Nachhaltig } \\
\text { Wirtschaften }\end{array}$ & & & & & & & & & $\mathrm{x}$ & $\mathrm{x}$ \\
\hline $\begin{array}{l}\text { Industrielle Kompetenzzentren }\left(\mathrm{K}_{\text {ind }}\right) \text {, } \\
\text { Kompetenznetzwerke }\left(\mathrm{K}_{\text {net }}\right)\end{array}$ & & & & & & & $\mathrm{x}$ & $\mathrm{x}$ & $\mathrm{x}$ & $\mathrm{x}$ \\
\hline E-Content & & & & & & & & & $\mathrm{x}$ & $\mathrm{x}$ \\
\hline BIOMED & & & & & & & & & & $\mathrm{x}$ \\
\hline TAKE OFF Aeronautics & & & & & & & & & & $\mathrm{x}$ \\
\hline Innovatives Bahnsystem (IBS) & & & & & & & & & & $\mathrm{x}$ \\
\hline $\begin{array}{l}\text { A3 Austrian Advanced } \text { Automotive } \\
\text { Technology }\end{array}$ & & & & & & & & & & $\mathrm{x}$ \\
\hline \multirow[t]{17}{*}{ FIT-IT } & & & & & & & & & & $\mathrm{x}$ \\
\hline & COST & $\mathrm{x}$ & $\mathrm{x}$ & $\mathrm{x}$ & $\mathrm{x}$ & $\mathrm{x}$ & $\mathrm{x}$ & $\mathrm{x}$ & $\mathrm{x}$ & $\mathrm{x}$ \\
\hline & EUREKA & $\mathrm{x}$ & $\mathrm{x}$ & $\mathrm{x}$ & $\mathrm{x}$ & $\mathrm{x}$ & $\mathrm{x}$ & $\mathrm{x}$ & $\mathrm{x}$ & $\mathrm{x}$ \\
\hline & $\begin{array}{l}\text { EU-Projektvorbereitung } \\
\text { bei KMUs }\end{array}$ & $\mathrm{x}$ & $\mathrm{x}$ & $\mathrm{x}$ & $\mathrm{x}$ & $\mathrm{x}$ & $\mathrm{x}$ & $\mathrm{x}$ & $\mathrm{x}$ & $\mathrm{x}$ \\
\hline & Holzforschung & & & $\mathrm{x}$ & $\mathrm{x}$ & $\mathrm{x}$ & $\mathrm{x}$ & & & \\
\hline & Lebensmittelinitiative & & & & & $\mathrm{x}$ & $\mathrm{x}$ & $\mathrm{x}$ & $\mathrm{x}$ & $\mathrm{x}$ \\
\hline & Mikrotechnik Österreich & & & & & & & & $\mathrm{x}$ & $\mathrm{x}$ \\
\hline & Nachwuchsförderung & $\mathrm{x}$ & $\mathrm{x}$ & $\mathrm{x}$ & $\mathrm{x}$ & $\mathrm{x}$ & $\mathrm{x}$ & $\mathrm{x}$ & $\mathrm{x}$ & $\mathrm{x}$ \\
\hline & Feasibility Studies & & & & & & $\mathrm{x}$ & $\mathrm{x}$ & $\mathrm{x}$ & \\
\hline & F\&E Dynamik & & & & & & $\mathrm{x}$ & $\mathrm{x}$ & $\mathrm{x}$ & \\
\hline & Start-up Förderung & & & & & & & & $\mathrm{x}$ & $\mathrm{x}$ \\
\hline & Foren für VC & & & & & & $\mathrm{x}$ & & & $\mathrm{x}$ \\
\hline & Technology Rating & & & & & & $\mathrm{x}$ & $\mathrm{x}$ & $\mathrm{x}$ & \\
\hline & TIN (JIISSY) & & & & & & & & $\mathrm{x}$ & \\
\hline & $\begin{array}{l}\text { PR-Offensive für } \\
\text { innovative KMU }\end{array}$ & & & & & & $\mathrm{x}$ & & & \\
\hline & LES & & & $\mathrm{x}$ & $\mathrm{x}$ & $\mathrm{x}$ & $\mathrm{x}$ & $\mathrm{x}$ & $\mathrm{x}$ & \\
\hline & TAFTIE & & & $\mathrm{x}$ & $\mathrm{x}$ & $\mathrm{x}$ & $\mathrm{x}$ & $\mathrm{x}$ & $\mathrm{x}$ & \\
\hline
\end{tabular}




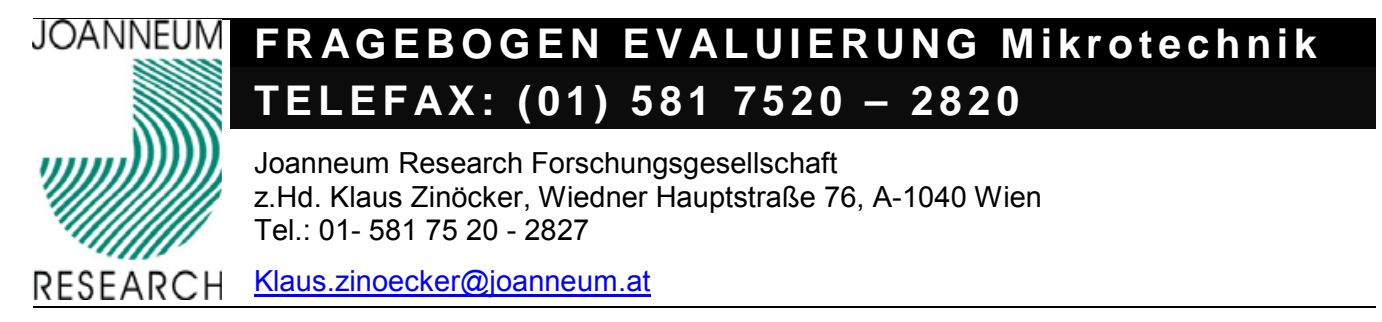

$\rightarrow$ Bitte senden Sie den Fragebogen bis zum 30. Juli 2004 zurück. Vielen Dank!

"Schwerpunktsetzung“: Hat Mikrotechnik aus Ihrer Sicht Zukunft, oder sollte man sich als Förderer auf andere Gebiete konzentrieren, etwa auf "Nano", „Life Sciences“ oder ähnliches?

„Rahmenbedingungen“: Haben sich die Rahmenbedingungen für die „Initiative Mikrotechnik“ seit 2000 wesentlich verändert? Wenn ja, wie? Und wie soll der FFF darauf reagieren?

"Instrumente“: Sind klassische FFF-Projekte ${ }^{1}$ genau das richtige Instrument, um mikrotechnische Aufgabenstellungen zu behandeln? Oder ist "Mikrotechnik" ein besonders riskanter Technologiebereich, in dem man mit anderen Instrumenten arbeiten sollte?

„Verbesserungen“: Welche Verbesserungen zur Optimierung der Initiative Mikrotechnik sind aus Ihrer Sicht vorzunehmen?

${ }^{1}$ Der FFF fördert mikro- und nanotechnische Projekte in der selben Art und Weise wie alle übrigen FFF-Projekte (Bottom-Up Förderung, Förderlaufzeit $1 \mathrm{Jahr}+$ Anträge für Verlängerung). 